\title{
CHATTER SUPPRESSION IN MACHINING PROCESSES
}

by.

\author{
Israr Ahmed Siddiqui \\ B.E. -Mechanical \\ NED University of Engineering \& Technology, Karachi
}

\author{
A thesis \\ Presented to Ryerson University \\ In Partial fulfillment of the \\ Requirement for the degree of \\ Master of Applied Science \\ in the program of \\ Mechanical Engineering
}

Toronto, Ontario, Canada, 2004

Copy right @Israr Siddiqui 2004 


\section{UMI Number: EC52976}

\section{All rights reserved \\ INFORMATION TO USERS}

The quality of this reproduction is dependent upon the quality of the copy submitted. Broken or indistinct print, colored or poor quality illustrations and photographs, print bleed-through, substandard margins, and improper alignment can adversely affect reproduction.

In the unlikely event that the author did not send a complete manuscript and there are missing pages, these will be noted. Also, if unauthorized copyright material had to be removed, a note will indicate the deletion.

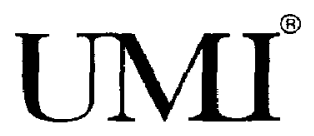

UMI Microform EC52976

Copyright 2008 by ProQuest LLC

All rights reserved. This microform edition is protected against unauthorized copying under Title 17, United States Code.

ProQuest LLC

789 East Eisenhower Parkway

P.O. Box 1346

Ann Arbor, MI 48106-1346 


\section{Author's Declaration}

I hereby declare that I am sole author of this thesis.

I authorize Ryerson University to lend this thesis to other institution or individuals for the purpose of scholarly research

Signature:

I further authorize Ryerson University to reproduce this thesis by photocopying or by other means, in total or in part, at the request of other institutions or individuals for the purpose of scholarly research.

Signature: 


\section{Borrower's Page}

Ryerson University requires the signatures of all persons using or photocopying this thesis. Please below, and give address and date

\begin{tabular}{|c|c|c|c|}
\hline Name & Signature & Address & Date \\
\hline & & & \\
\hline & & & \\
\hline & & & \\
\hline & & & \\
\hline & & & \\
\hline & & & \\
\hline & & & \\
\hline & & & \\
\hline & & & \\
\hline & & & \\
\hline & & & \\
\hline & & & \\
\hline & & & \\
\hline & & & \\
\hline & & & \\
\hline & & & \\
\hline
\end{tabular}




\begin{abstract}
The development of an untended machining system has been the subject of research for quite some time. Today, the need for such a system is greater than it once was because of the shortage of skilled workers, higher machining speeds, increase in precision machining, and the need to lower downtime. One aspect of machining process has been under investigation is tool chatter.
\end{abstract}

Chatter is a machining instability resulting from self-excited vibration caused by interaction of the chip removal process, the cutting tool, and the structure of the machine tool. Chatter can severely reduce the material rate by putting limits to cutting speed and width of cut.

This thesis describes a novel approach for active, on line suppression of chatter in machining operations. The goal of chatter suppression is to minimize the chatter amplitude and therefore extend the chatter stability boundary. Once the presence of chatter is detected the suppression system will be activated. A neural network model is used to calculate current gradient values with respect to the parameters of the active vibration source. This gradient information will be used by an optimization module to find the optimal set of parameters for the active vibration source. The methodology described is evaluated through simulation studies and simulation results confirmed the effectiveness of the approach. 


\section{Acknowledgements}

The authors wish to express his sincerest gratitude to his thesis supervisor for his support and guidance of this research, Prof. Ahmad Ghasempoor.

I also would like to thank the Department of Mechanical and Industrial Engineering and the school of Graduate Studies at Ryerson University for their support in terms of department facilities, research stipend and scholarship.

A special thanks goes out to my friends Syed Kashif Ali, Bijal Shah and Sadeep Dhar for their advice, experience and knowledge with regard to chatter simulation.

I would like to acknowledge my supervisors funding resource- Natural Sciences and engineering Research Council of Canada (NSERC) for the financial support provided for this research.

Finally, I would like to thank my wife for her support and understanding throughout the duration of this work. 


\section{Table of Contents}

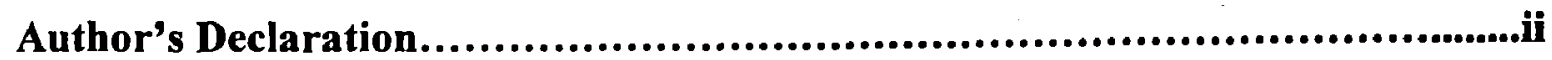

Borrower's Page.......................................................................ii

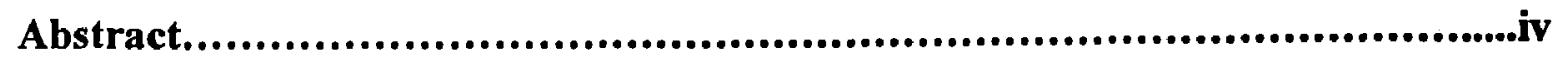

Acknowledgements..................................................................v

Table of Contents....................................................................vi

List of Figures...................................................................

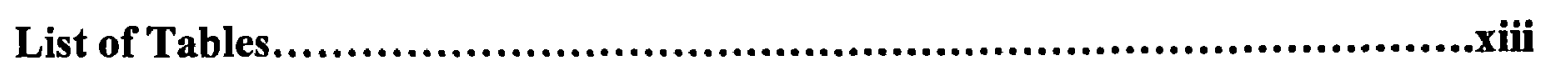

Nomenclature.......................................................................xiv

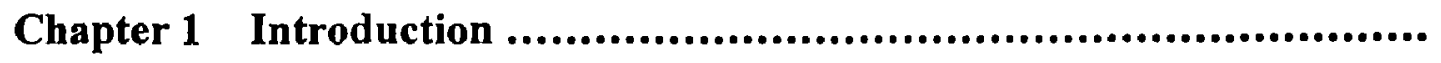

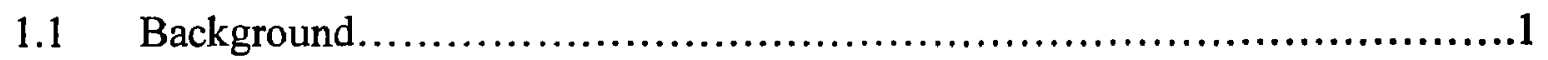

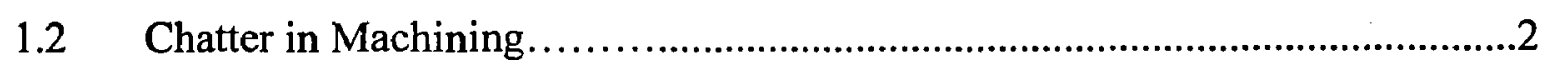

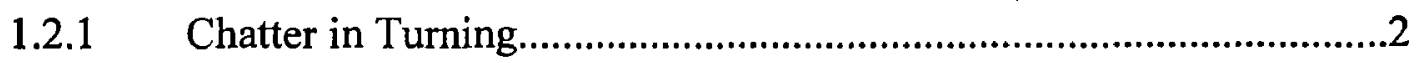

1.2.2 Chatter in Milling.........................................................................

1.3 Examples of Chatter.........................................................

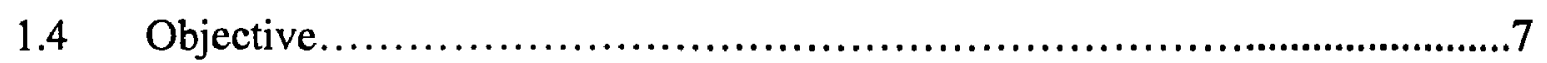

Chapter 2 Literature Review

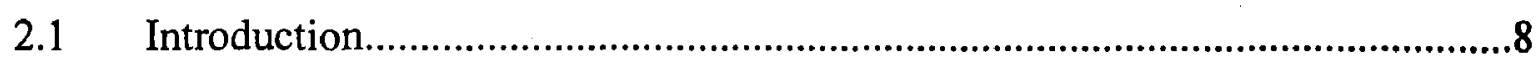

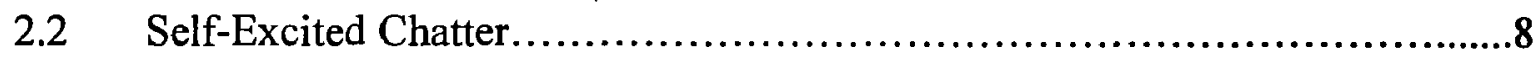

$2.3 \quad$ Regeneration of Waviness...............................................11

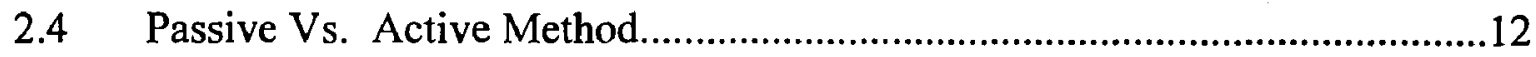

2.4.1 Regulated Spindle Speed..........................................................13

2.4.2 Viscoelastic Dynamic Damping.....................................16

2.4.3 Variable Cutting.....................................................19

2.4.4 Vibration Absorber..................................................25

2.4.5 Viscous Damper.............................................28

2.4.6 Actuators with Inertia Mass.....................................29 


\section{Chapter 3 Methodology}

Introduction....................................................... 32

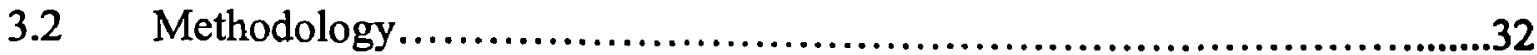

3.3 Background on Neural Networks...........................................38

3.3.1 Multilayer Feed Forward Neural Networks............................... 38

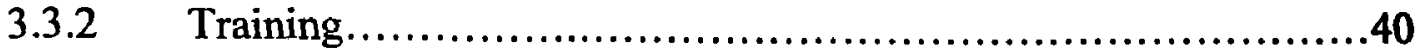

3.3.3. Gradients of a Neural Network......................................41

3.3.4 Validation....................................................42

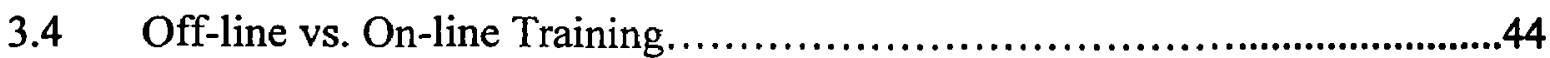

3.4.1 Dynamic vs. Static Neural Network................................................44

3.5 Extracting gradient information from a Neural Network Model...............46

3.6 Signal Processing............................................................

3.7 Maximum Entropy (all poles) Method.................................51

3.8 Overview of the proposed method.........................................52

\section{Chapter 4 Simulation Studies ................................................}

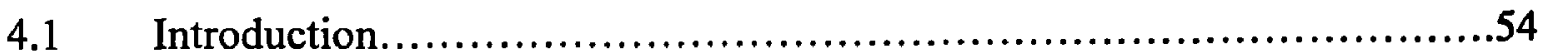

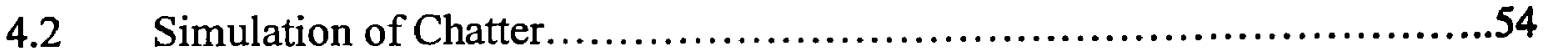

$4.3 \quad$ Simulation Model for Turning ..........................................58

4.4 Chatter Simulation in Milling.........................................64

4.5 Simulation Model for Milling......................................65

\section{Chapter 5 Results}

5.1 Introduction........................................................

5.2 Chatter Suppression in Turning ........................................71

5.2.1 Neural Network Architecture ..............................................71

5.2.2 Off-Line Training...............................................74 
5.2.3 Training and Test Set.......................................75

5.2.4 Chatter Suppression System........................................76

5.2.5 Simulation Results...........................................78

5.3 Chatter Suppression in Milling........................................... 81

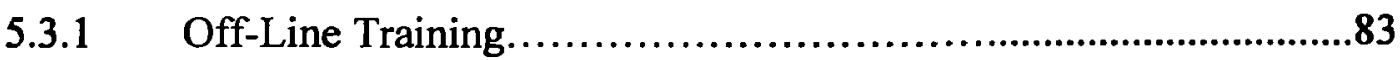

5.3.2 Simulation Results................................................

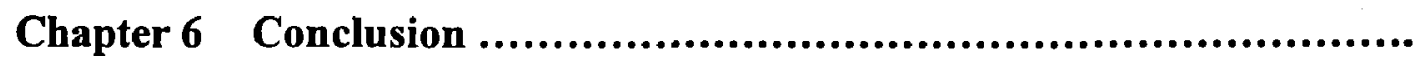

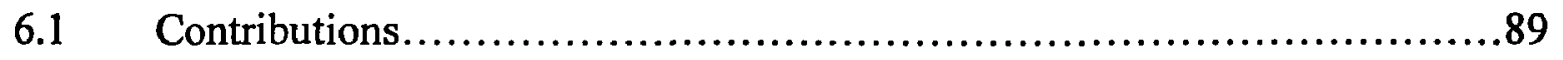

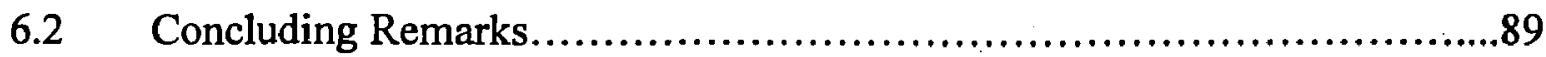

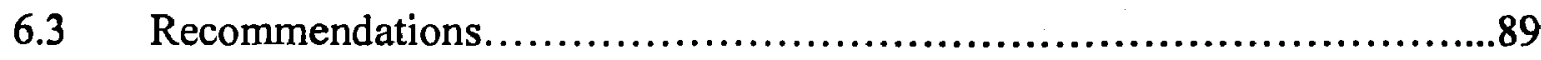

REFERENCES............................................................ 91

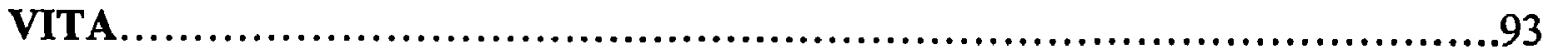




\section{List of Figures}

Figure 1.1: Cylindrical turning on a lathe (boothroyd and Knight, 1989)................3

Figure 1.2: Turning: Chip width b..............................................4

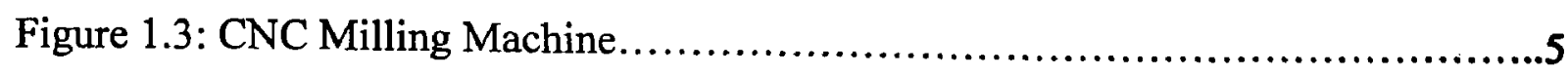

Figure 1.4: Milling: Cumulative chip width for all teeth cutting simultaneously...........5

Figure 1.5: Wavy surface of work piece after chatter (Chiriacesu, 1990) ..................6

Figure 1.6: Wavy surface of a hollow cylinder work piece after chatter..................7

Figure 2.1: Self-excited vibration...............................................10

Figure 2.2: Chip thickness variation between tool and the work piece..................11

Figure2.3: Closed loop representation of machining ...............................12

Figure 2.4: Block diagram of the regenerative chatter.............................14

Figure 2.5: Simulation of the placement $y(t)$ for (a) without control, and under control with (b) $v=0.25$, (c) $v=0.4$, (d) $v=0.1$, (e) $v=0.75$, and (f) $v=1 \ldots \ldots \ldots \ldots \ldots \ldots \ldots . .15$

Figure 2.6: Experimental setup...................................................16

Figure 2.7: Excitation Methods................................................... 18

Figure 2.8 Installation of the dynamic damper on the tool post and its dimensions......19

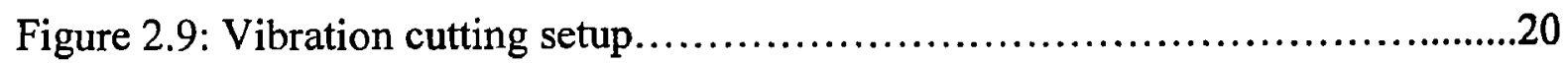
1.

Figure 2.10: Work holder and measurement setup for work displacement............21

Figure 2.11: Experimental work displacement with tool rake angle $\alpha_{0}=9^{\circ}$, tool

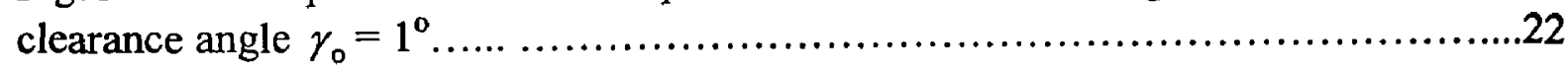

Figure 2.12: Experimental work displacement with tool rake angle $\alpha_{0}=6$, tool

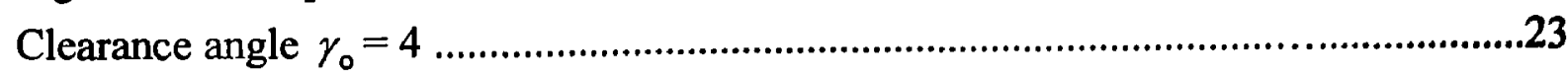

Figure 2.13: Experimental work displacement with tool rake angle $\alpha_{0}=3^{\circ}$, tool clearance

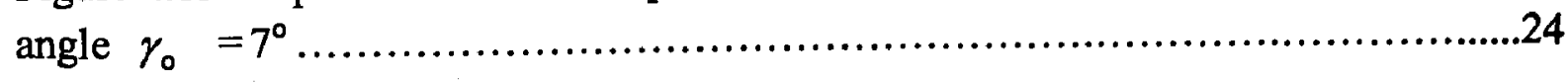


Figure 2.14: Experimental work displacement with tool rake angle $\alpha_{0}=0^{\circ}$, tool clearance

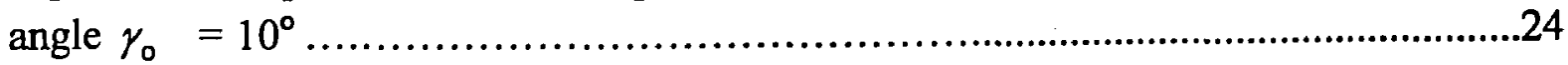

Figure 2.15: Control block diagram for chatter in turning ................................25

Figure 2.16: A simplified response function of the cutting tool (a) real part;

(b) magnitude.

Figure 2.17: Simulated stability chart: (a) chatter frequency versus spindle speed

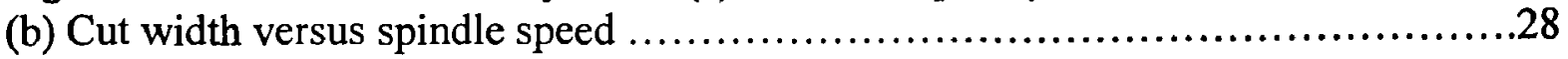

Figure 2.18: Configuration of a beam with replicated internal viscous dampers.

Figure 2.19: Frequency response function between the input voltage and output force of the piezoelectric actuator with an inertial mass of $150 \mathrm{~g}$ (a) magnitude;

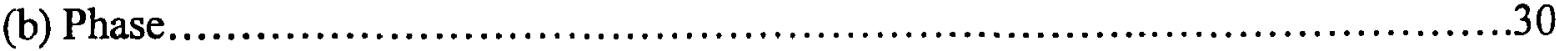

Figure 2.20: A simplified model for the cutting tool with the piezoelectric actuator....31

Figure 3.1: Active Chatter suppression system..................................31

Figure 3.2: Simplified model of cutting process................................32

Figure 3.3: Machining Process as an input/output system.........................33

Figure 3.4: Chatter Suppression System.......................................34

Figure 3.5: Steepest Descent Method...........................................35

Figure 3.6: Active Chatter Suppression System.................................36

Figure 3.7: Typical multiplayer feed forward neural network architecture ............38

Figure 3.8: Neurons with multiple inputs and a single output ....................38

Figure 3.9: Training and test error as a function of training iterations ...............42

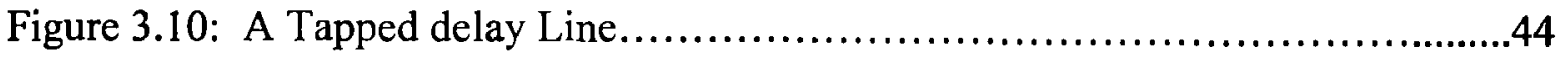

Figure 3.11: Dynamic neural networks with tapped delayed line....................48

Figure 3.12: Reprsentation of frequency response using finite number of bins.

Figure 3.13: Adaptive feed forward chatter suppression system.....................51 
Figure 4.1: Simplified model of cutting process.

Figure 4.2: The regeneration diagrams relating to force, surface waviness and vibration

Figure 4.3(a): Model for simulation of turning.....................................58

Figure 4.3(b): Model for simulation of turning....................................59

Figure 4.4: Turning Chatter Simulation for $b=1.63 \mathrm{~mm} \ldots \ldots \ldots \ldots \ldots \ldots \ldots \ldots \ldots \ldots . . \ldots 2$

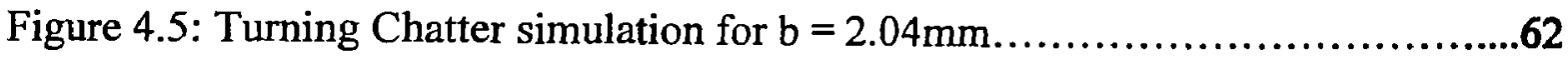

Figure 4.6: Turning Chatter Simulation for $b=2.45 \mathrm{~mm} \ldots \ldots \ldots \ldots \ldots \ldots \ldots \ldots \ldots \ldots . \ldots \ldots$

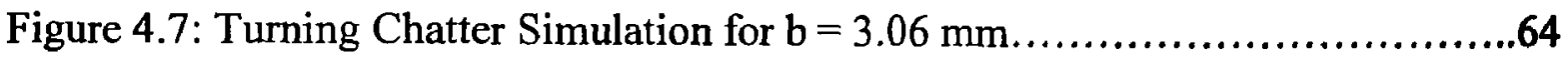

Figure 4.8: End Milling Process..............................................65

Figure 4.9: Milling cutter with 8 teeth in a slotting cut...........................66

Figure 4.9(a): Milling Chatter Simulation for $b=1.00 \mathrm{~mm} \ldots \ldots \ldots \ldots \ldots \ldots \ldots \ldots \ldots$

Figure 4.9 (b): Milling Chatter Simulation for $b=1.00 \mathrm{~mm} \ldots \ldots \ldots \ldots \ldots \ldots \ldots \ldots \ldots . \ldots . \ldots . \ldots \ldots$

Figure 4.10(a) : Milling Chatter Simulation for $b=2.00 \mathrm{~mm} \ldots \ldots \ldots \ldots \ldots \ldots \ldots \ldots \ldots . \ldots 6$

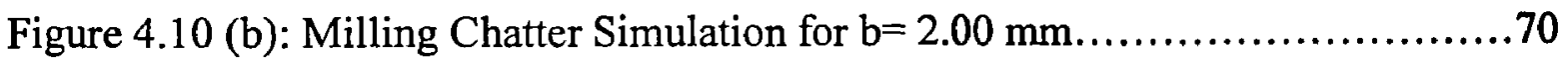

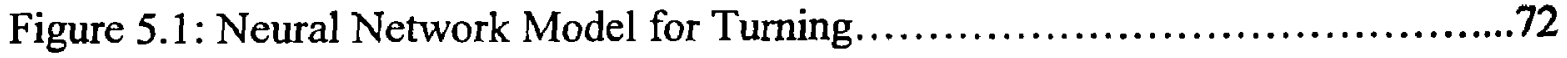

Figure 5.2: Simplified Cutting Process with Two Actuators.......................73

Figure 5.3: Sampling Period with Maximum Amplitude..........................73

Figure 5.4: Sigmoid Function................................................. 74

Figure 5.5: Neural Network with Varying Number of Hidden Nodes..................76

Figure 5.6: Block Diagram of Chatter Suppression System........................77

Figure 5.7: Tool chatter with active suppression system Off......................78

Figure 5.8: Frequency response of the tool with active suppression system off........79

Figure 5.9: Tool vibration with active suppression system On.....................80 
Figure 5.10: Frequency response of the tool with active suppression On.

Figure 5.11: Comparison of frequency response of the tool with active suppression On and Off.

Figure 5.12: Neural Network Model for Milling.................................82

Figure 5.13: Simplified Milling Cutting Process with Two Actuators................83

Figure 5.14: Neural Network with Varying Number of Hidden Nodes..............84

Figure 5.15: Tool chatter with active suppression system Off.......................85

Figure 5.16: Tool chatter with active suppression system Off......................86

Figure 5.17: Frequency response of the tool with active suppression system off.......86

Figure 5.18: Tool vibration with active suppression system On....................87

Figure 5.19: Tool vibration with active suppression system On....................87

Figure 5.20: Comparison of frequency response of the tool with active suppression

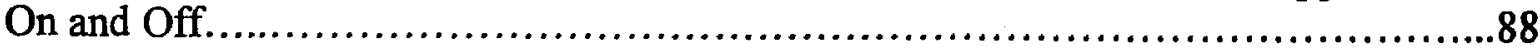




\section{List of Tables}

Table 4.1: Cutting Parameters......................................................61

Table 4.2: Cutting Parameters used in milling simulation.............................68 


\section{Nomenclature}

\section{Acronym}

d

E

f

i

$\mathrm{NN}$

$\mathrm{O}$

OTF

W

y

$\mathbf{z}$

A

$\mathbf{d}_{\mathbf{i}}$

$\mathrm{d}_{0}$

$\mathrm{E}_{\mathbf{a}}$

E

K

L

$\mathbf{P}$
Definition

Desired Output

Error (between desired and actual output)

Neural Network Function

All output nodes in the network

Neural Network

Actual output node

Oriented transfer function

Neural network weight

Output

Input

Area of cross section

Inner diameter

Outer diameter

Apparent complex elastic modulus

Elastic modulus

Stiffness

Length

Preload, Prestrain 
b

$b_{\text {lim }}$

$\mathrm{K}_{\boldsymbol{s}}$

$\mathrm{h}_{\mathrm{av}}$

$h_{m}$

$\mathrm{m}$

n

$\mathbf{F}_{\mathrm{T}}$

$\mathrm{F}_{\mathrm{N}}$

Zold
Chip width

Limit value of chip width

Specific force

Average chip thickness

Mean chip thickness

Mass

Spindle speed

Tangential force

Normal Force

Displacement 


\section{Introduction}

\section{Chapter 1}

\subsection{Background}

The manufacturing industry has a significant impact on the Canadian economy, as it comprises a significant portion of the total GDP in Canada-(12.88\% in 1998) [1]. More specially, the province of Ontario is home to assembly plants representing six different automotive manufacturers: Ford, General Motors, Daimler Chrysler, Honda, Toyota and CAMI (a joint venture between GM and Suzuki). Parts manufacturers and suppliers also have plants spread throughout the province.

In the global marketplace, a company must be able to produce a quality component at a reasonable price to stay in operation. Compliance with quality standards such as ISO 9001 and QS-9000 is now required for manufacturers to compete internationally. To remain competitive, companies must find ways of not only improving part quality, but also reducing costs.

Machining operations are often claimed to be the most important processes in engineering manufacture. This is based on the fact that some machining is involved in the production of almost any item. From the point of view of expenditure, machining is of considerable importance. It is estimated that in the United States alone some $\$ 10$ billion annually is spent on machining processes [1].

Since the late 1940 's, the whole approach to the shaping processes has undergone a number of revisions. Casting to final shape has become reasonably commonplace; accurate forming has been accepted, with considerable development in processes such as spinning, extrusion and high- rate forming. These developments in the so-called chipless production processes have been said to herald the end of material removal processes. In fact this does not seem likely. There are situations where non-chip-forming 
applications are economically desirable, but for a wide range of conditions the simplicity of the machining process will make it an economical method for years to come. In keeping with the forming processes, great developments have been made in material removal processes in recent years. Tool materials have been improved. The size and speed of machine tools have increased considerably. Machine-tool control has been improved.

\subsection{Chatter in Machining}

Chatter is a machining process instability resulting from self-excited vibration caused by the interaction of the chip removal process and structure of the machine tool. These vibrations inhibit higher metal removal rates (MRR), accelerate tool wear, and result in poor surface finish and even damage to machine components. It is estimated that higher MMR achievable through chatter control could yield more than a $\$ 10$ billion increase in US GDP. Often the onset of machining instability, namely chatter, occurs at less severe cutting conditions than those associated with breakage. This is true especially when machining flexible parts such as turbine blades or when machining deep features using slender end mills [2].

\subsubsection{Chatter in Turning}

Turning is a single point machining process, which is used to machine casting, forging, and other work pieces into cylindrical components, which require precision dimensional tolerance and good surface finish (Figure 1.1). There are several parameters affecting onset conditions in turning. For a given work piece (material, geometry, damping, boundary conditions), a given lathe (tool structure, support, geometry, and insert), the parameters are cutting speed (or spindle spin rate), feed rate and depth of cut. Cutting speed is the rate at which the cutting tool removes the material from the work piece. Feed rate is the advancement rate of the cutting tool along the work piece per revolution. Depth of cut is the thickness of the metal removed in the radial direction [3]. 


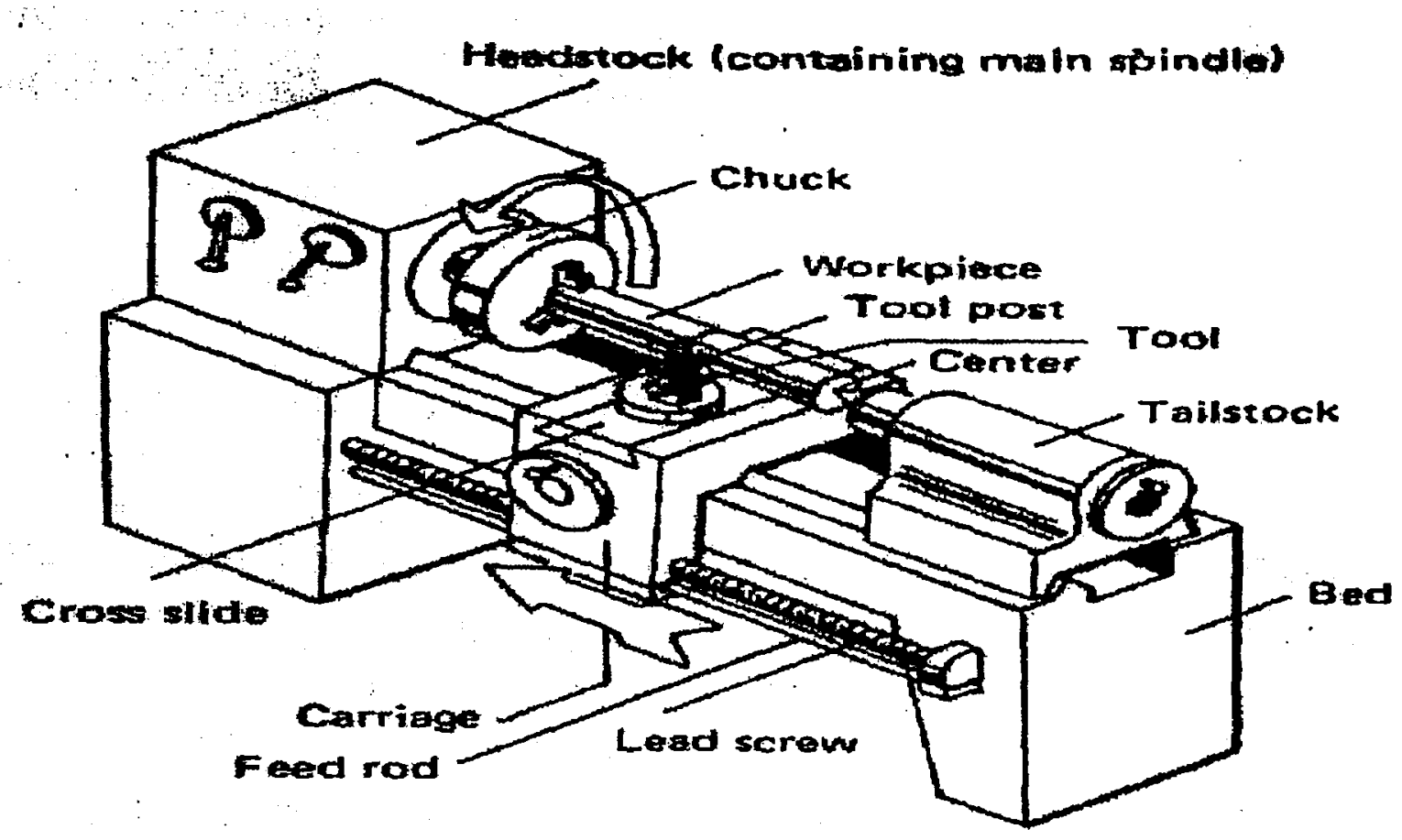

Figure 1.1: Cylindrical turning on a lathe (boothroyd and Knight, 1989)[4]

In turning operations, chatter is a dynamic instability of the cutting process, which results from the interaction of the metal cutting process and structural dynamics of the machine tool. Under certain cutting conditions, the machine tool and work piece dynamical system becomes unstable, which leads to chatter.

Chatter and noise in metal cutting are ubiquitous problems in the workshop. The turning operation is one kind of metal cutting that exhibits chatter related problems. Severe noise is also a problem due to regulations in preventing hearing loss.

The most significant cutting parameter, which is decisive for the generation of chatter, is the width of cut $b$ (Figure 1.2). For sufficiently small chip widths, cutting is stable, without chatter. By increasing $b$, chatter starts to occur at a certain width $b_{\text {lim }}$ and becomes more energetic 
for all values of $b<b_{\text {lim. }}$. The value of " $b_{\text {lim }}$ " depends on the dynamic characteristics of the structure, on the work piece material, cutting speed and feed, and on the geometry of the tool [5]

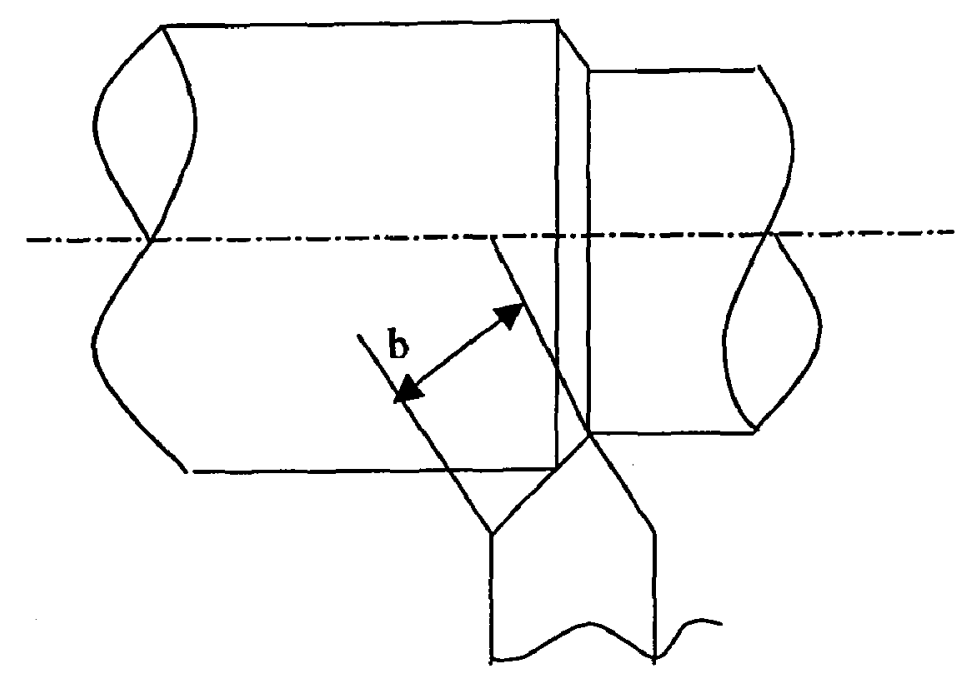

Figure 1.2: Turning: Chip width $b$ [5]

Regeneration of waviness is possible because in almost all machining operations the tool removes the chip from the surface that was produced by the tool in the preceding pass, that is, the surface produced in turning during the proceeding revolution. If there is relative vibration between the tool and the work piece waviness it is generated on the cut of the surface.

\subsubsection{Chatter in Milling}

Milling is a basic machining process by which a surface is generated by progressive chip removal (Figure 1.3). Two broad categories of milling processes are:

1. Peripheral milling (slab milling): the teeth located on the periphery of the cutter body generate the surface and the surface is parallel with the axis of rotation of the cutter. 
2. Face milling (end milling): the surface generated is at right angles to the cutter axis.

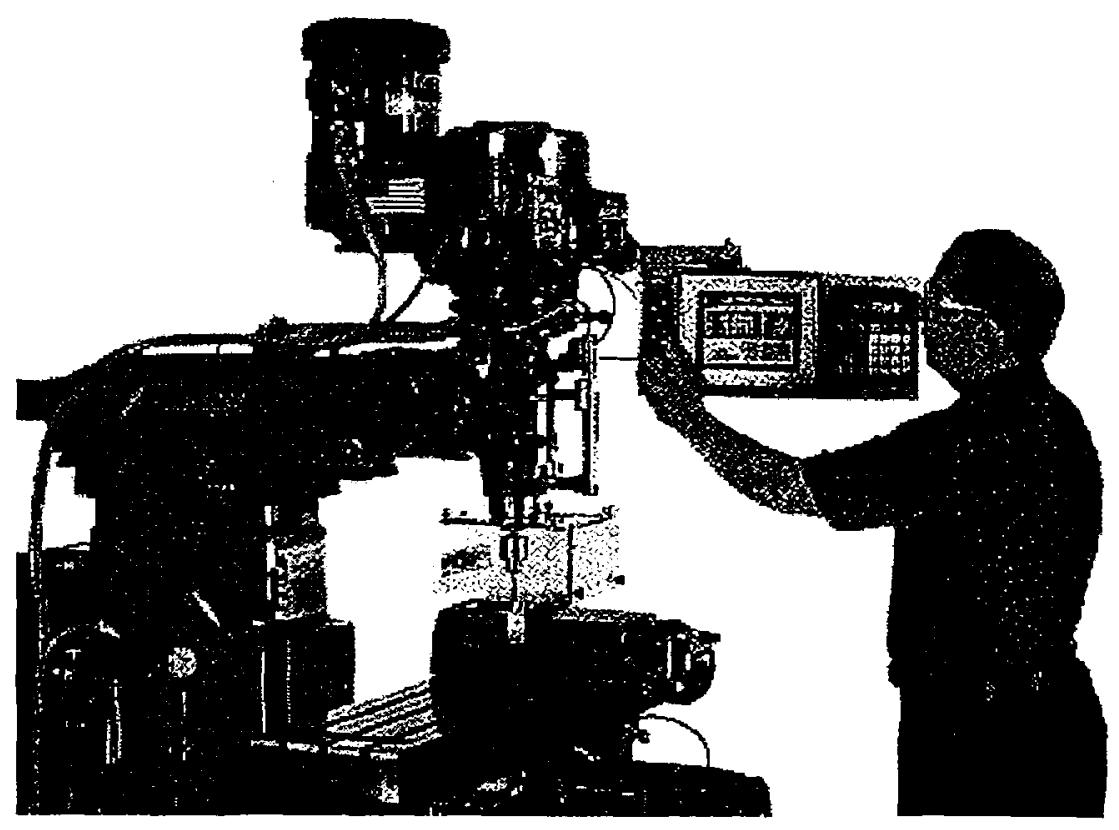

Figure 1.3: CNC Milling Machine

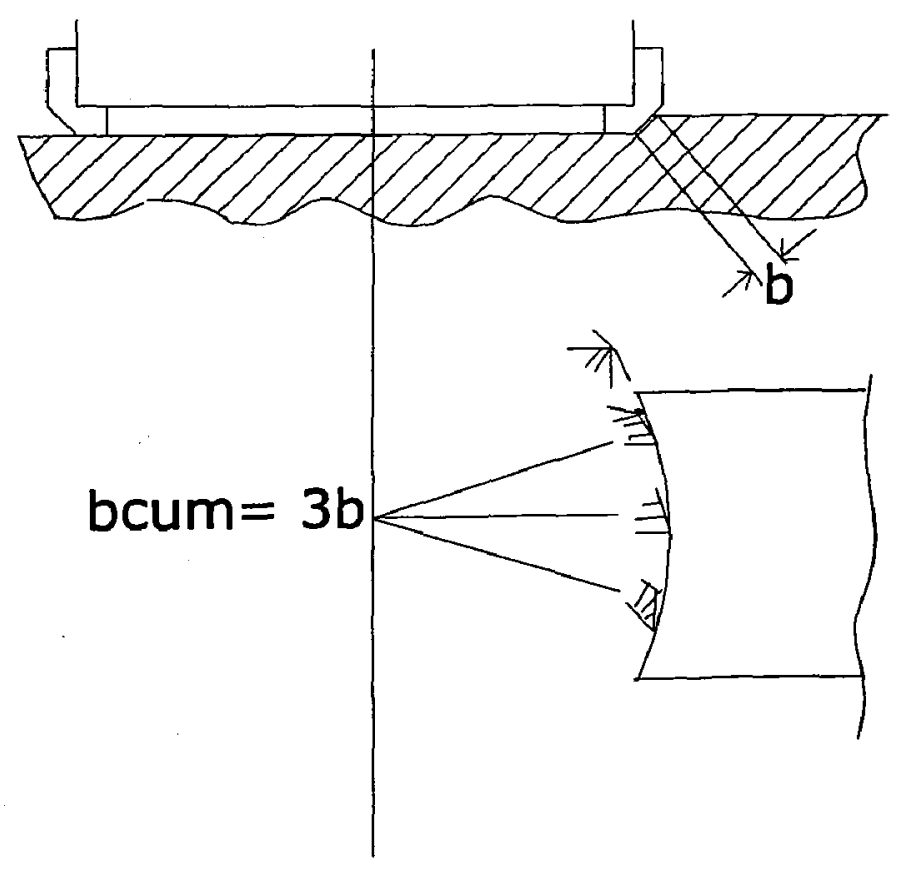

Figure 1.4: Milling: Cumulative chip width for all teeth cutting simultaneously [5] 
Chatter in milling is more complex than in turning because the system of forces on the individual teeth rotates with the directions of flexibilities of the vibratory system. In milling, the cumulative chip width $b_{\text {cum }}$ (Figure 2.4) has to be considered, which is the sum of the chip widths of all the teeth cutting simultaneously.

In all machining operations, the tool removes the chip from the surface that was produced by the tool in the preceding pass, that is, the surface produced in one preceding tooth of the cutter.

\subsection{Examples of Chatter}

Chatter is undesirable because of its adverse effects on surface finish, machining accuracy, and tool life.

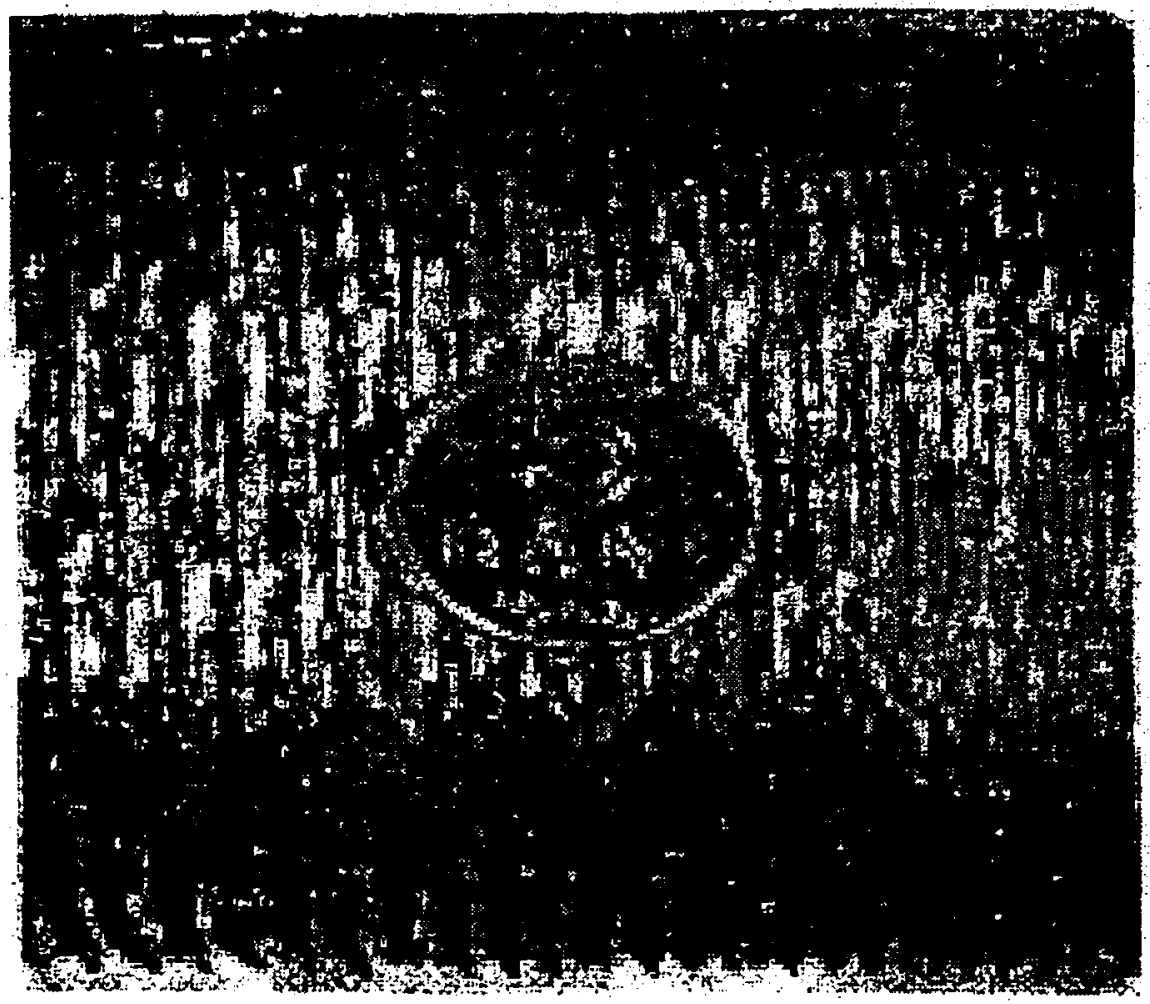

Figure 1.5: Wavy surface of work piece after chatter (Chiriacesu, 1990) [6] 
Furthermore, chatter is also responsible for reducing output because, if no remedy can be found, metal removal rates have to be lowered until vibration-free performance is obtained. One important aspect of chatter is that it occurs suddenly. As a result, significant damage may occur before any action can be taken. Figures 1.5 and 1.6 illustrate the effects of chatter on work piece surface finish.

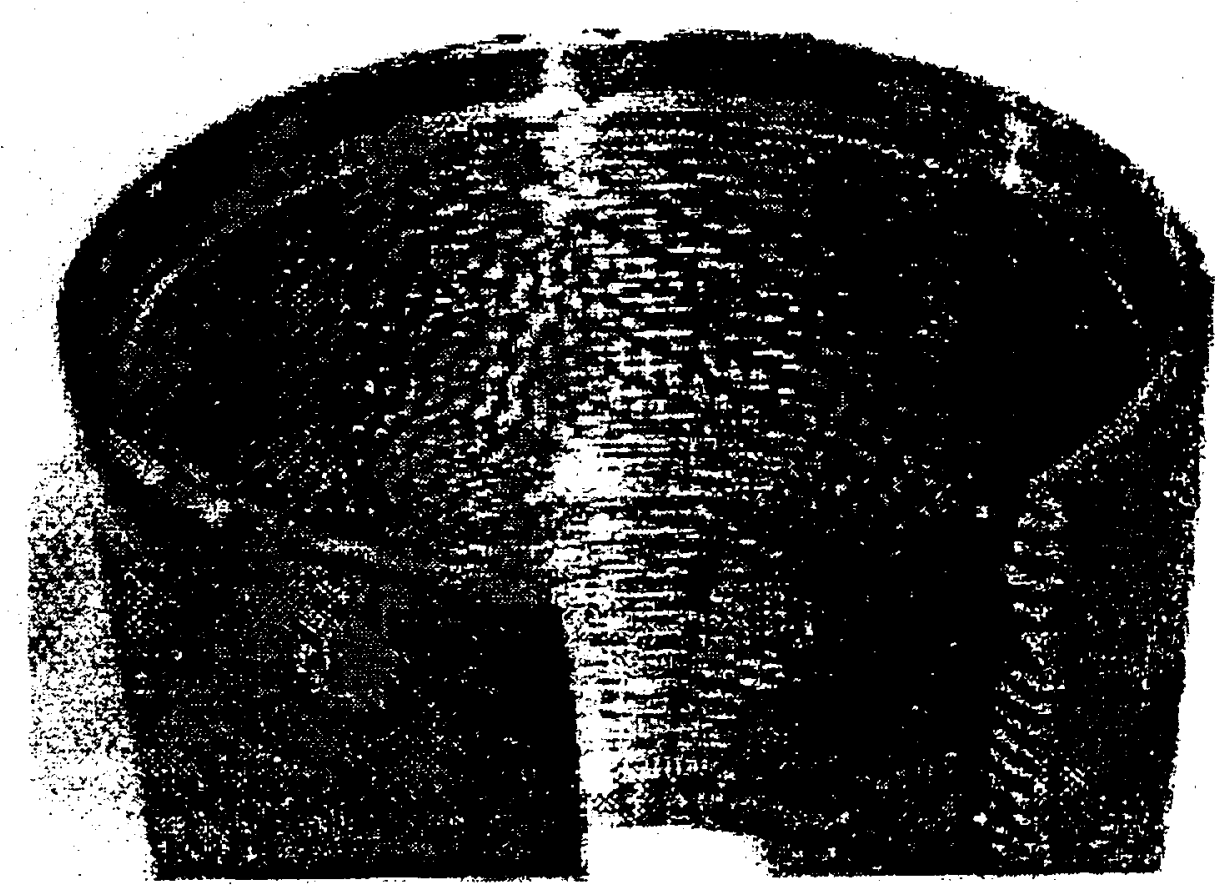

Figure 1.6: Wavy surface of a hollow cylinder work piece after chatter [6]

\subsection{Objective}

The objective of this work is to develop a novel approach for active, on-line suppression of chatter in machining operations. The goal of chatter suppression is to minimize the chatter amplitude and therefore extend the chatter stability boundary. 


\section{Chapter 2}

\section{Literature Review}

\subsection{Introduction}

Chatter, a violent relative vibration between the work piece and the cutting tool, is a frequent problem in machining operations. Chatter affects the productivity, the surface finish, and the tool life. It also causes severe acoustic noise in the work environment. To reduce chatter, approaches such as continuously varying the spindle speed, using a damped absorber, and increasing the stiffness of the machine structure have been studied. Chatter falls into two categories, forced chatter and self-excited chatter. Forced chatter is caused by external forces and it is usually not a problem in machining because the external forces or the dynamic compliance of the machine structure can be reduced to permissible values once the external forces causing the chatter are identified. However, self-excited chatter is a serious problem, because its amplitude increases with the progress of cutting [5].

\subsection{Self-Exited Chatter}

Chatter is a self- excited vibration that occurs in metal cutting if the chip width is too large with respect to the dynamic stiffness of the system. This vibration occurs when a steady input of energy is in some way modulated into vibration. Self-excited vibrations usually result from a dynamic instability of the cutting process. This phenomenon is commonly referred to as machine tool chatter. Under such conditions these vibrations start and quickly grow (Figure 2.1). If large tool-work engagements are attempted, oscillations suddenly build up in the structure and effectively limit metal removal rates. The structure oscillates in one of the natural modes of vibration. 
The cutting force becomes periodically variable, reaching considerable amplitudes, the machined surface becomes undulated, and the chip thickness varies in the extreme so much that it becomes dissected (Figure 2.2).

\section{Self-Excited Vibration}

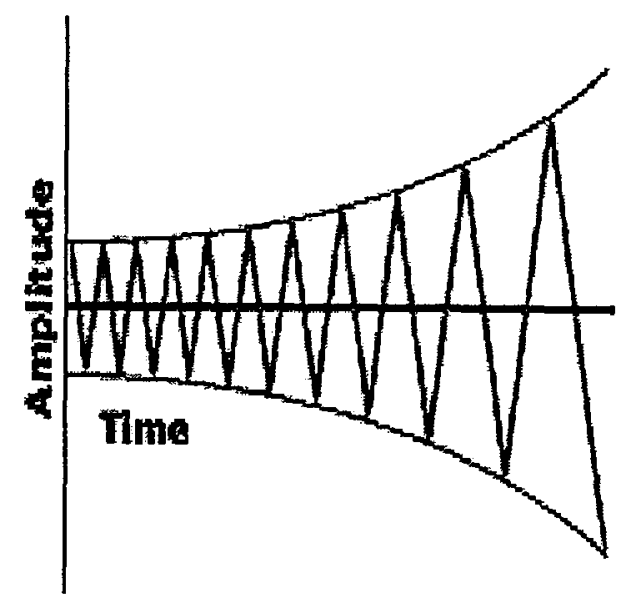

- Self-generated from a steady energy source

- Example: violin, chatter

Figure 2.1: Self-excited vibration [5]

In the turning and milling operations, chatter or vibration is a frequent problem, which affects the result of the machining, and, in particular, the surface finish. Tool life is also influenced by vibration. Severe acoustic noise in the working environment frequently occurs as a result of dynamic motion between the cutting tool and the work piece [7]. In all cutting operations like turning, boring and milling, vibrations are induced due to the deformation of the work piece. This implies several disadvantages, economical as well as environmental.

In the turning operation, the tool and tool holder shank are subjected to a dynamic excitation due to the deformation of work material during the cutting operation. The stochastic chip formation process usually induces vibrations in the machine-tool system. Energy from the chip formation process excites the mechanical modes of the machine- 
tool system. Modes of the work piece may also influence the tool vibration [7]. The relative dynamic motion between cutting tool and work piece will affect the result of the machining, in particular the surface finish.

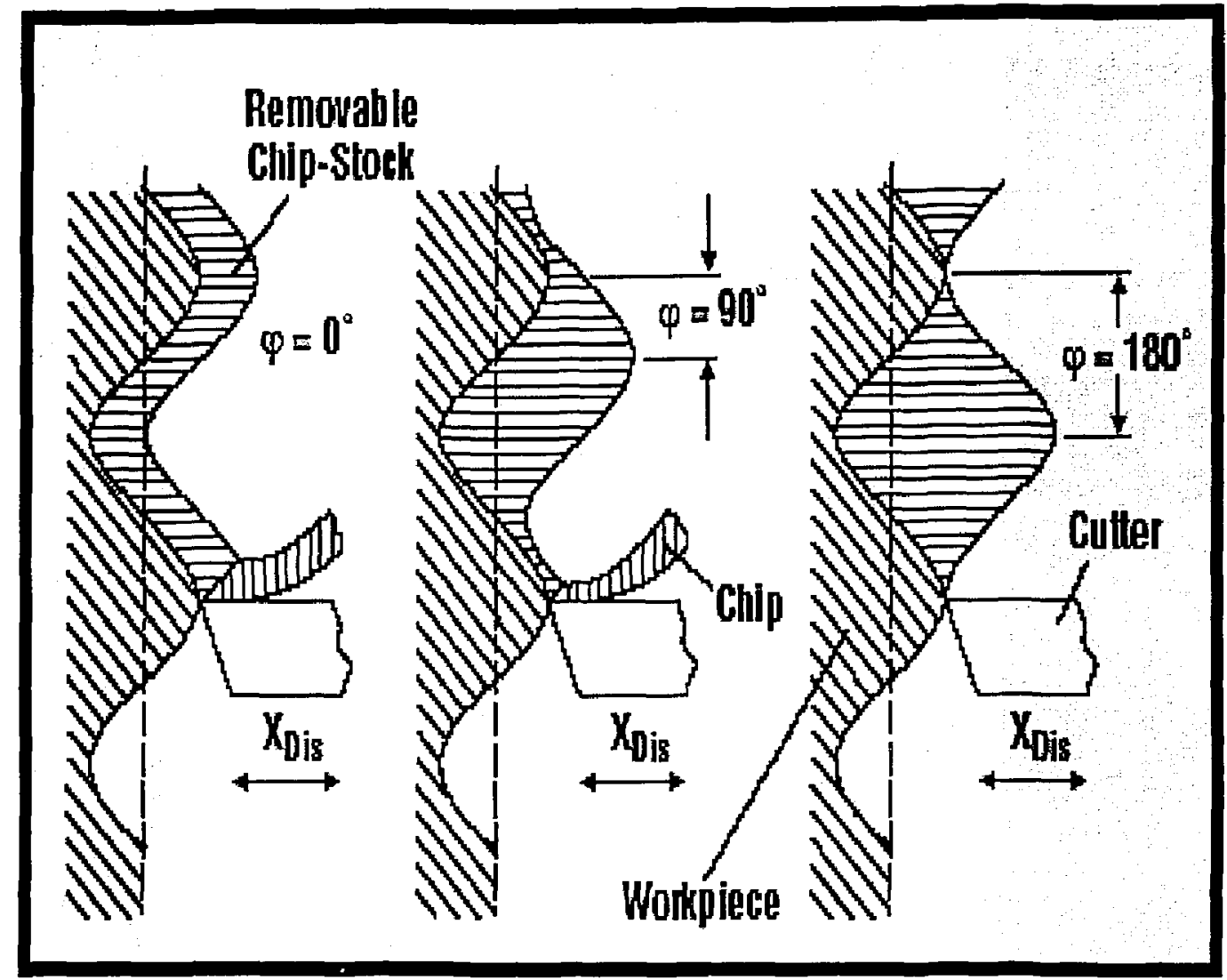

Figure 2.2: Chip thickness variation between tool and the work piece[5]

Furthermore, the tool life is correlated with the amount of vibrations and the acoustic noise introduced. The noise level is sometimes almost unbearable. The most significant cutting parameter, which is decisive for generation of chatter, is the width of cut. For sufficiently small chip widths, cutting is stable, without chatter. By increasing $b$, chatter starts to occur at a certain width and becomes more energetic for all values of $b>b_{\text {lim. }}$. The value of $b_{\text {lim }}$ depends in the dynamic characteristics of the structure, on the 
work piece material, cutting speed and feed, and on the geometry of the tool. There are two main sources of self-excitation in metal cutting [5]:

1. Mode coupling

2. Regeneration of Waviness

\subsection{Regeneration of Waviness}

Most chatter occurring in practical machining operations is regenerative chatter. It is caused by the undulation of a work piece surface. The natural frequency of regenerative chatter is around the natural frequency of the machining system. Regenerative chatter is so named because of the closed-loop nature of this interaction (Figure 2.3). Each tooth pass leaves a modulated surface on the work piece due to the vibrations of the tool and work-piece structures, causing a variation in the expected chip thickness (Figure 2.2) Under certain cutting conditions (i.e., feed, depth, and speed), large chip thickness variations, and hence force and displacement variations, occur and chatter is present [8].

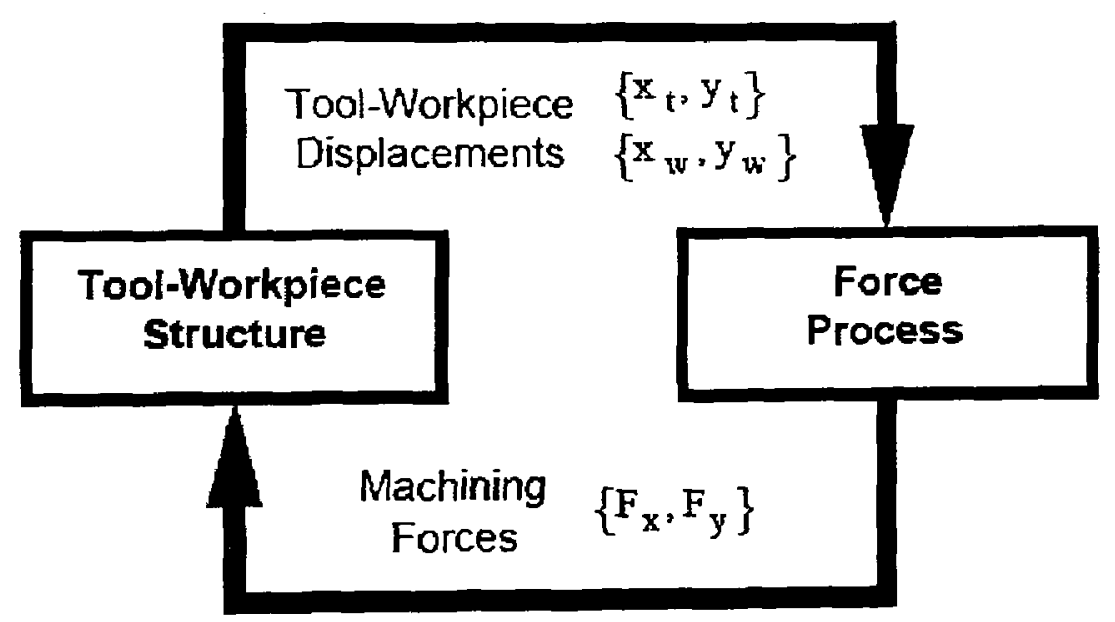

Figure 2.3: Closed loop representation of machining [8]

Either passive or active methods are used to suppress chatter vibration are: 


\subsection{Passive vs. Active Method}

Usually passive methods suppress chatter by using energy absorbing dampers to consume energy absorbing dampers to consume the energy input or by changing the cutting conditions to reduce the energy input or by changing the cutting conditions to reduce energy generated during the machining process. Other passive approaches include the increase of the rigidity of the machine tool structure by redesigning or through the use of dampers, changed tool geometry, variation of directional factors, etc. Passive methods suffer from the fact that as soon as the machine tool-tool work piece configuration changes, the machines have to be tuned. On the other hand, active control approaches provide numerous advantages: e.g., improved low frequency performance, reduction of size and weight, and programmable flexibility of design. There is a limit to the improvement which can be achieved in chatter behavior of cutting tools through passive methods; for higher speeds, smaller tolerances, and greater process flexibility and efficiency, these methods have proven less than adequate. Some of the more important active chatter suppression methods are reviewed here.

\subsubsection{Regulated Spindle Speed}

Lio et al [9] proposed a new online control method to suppress chatter during the machining process by regulating spindle speed. The basic principal is to consume energy by the best vibration phase. There is no need to halt the feed during the change of speed, and hence tool life can be increased and good surface finish can be obtained. Since the computer can monitor the current vibration state and send out new speed commands in real time, this control approach can achieve on-line control of the time varying and nonlinear cutting process. If the dynamic behavior of the cutting process is neglected, and assuming that the cutting force is proportional to the instantaneous uncut chip thickness, then the regenerative chatter can be simplified by a block diagram as in Figure 2.4 [10]. The dynamic cutting force signal collected from a dynamometer is passed through a low pass filter, and then digitized. The Fast Fourier transform is carried out to obtain the corresponding power spectrum. The chatter is identified when the intensity at a certain 
frequency other than the spindle speed and tooth passing frequency exceeds a critical value.

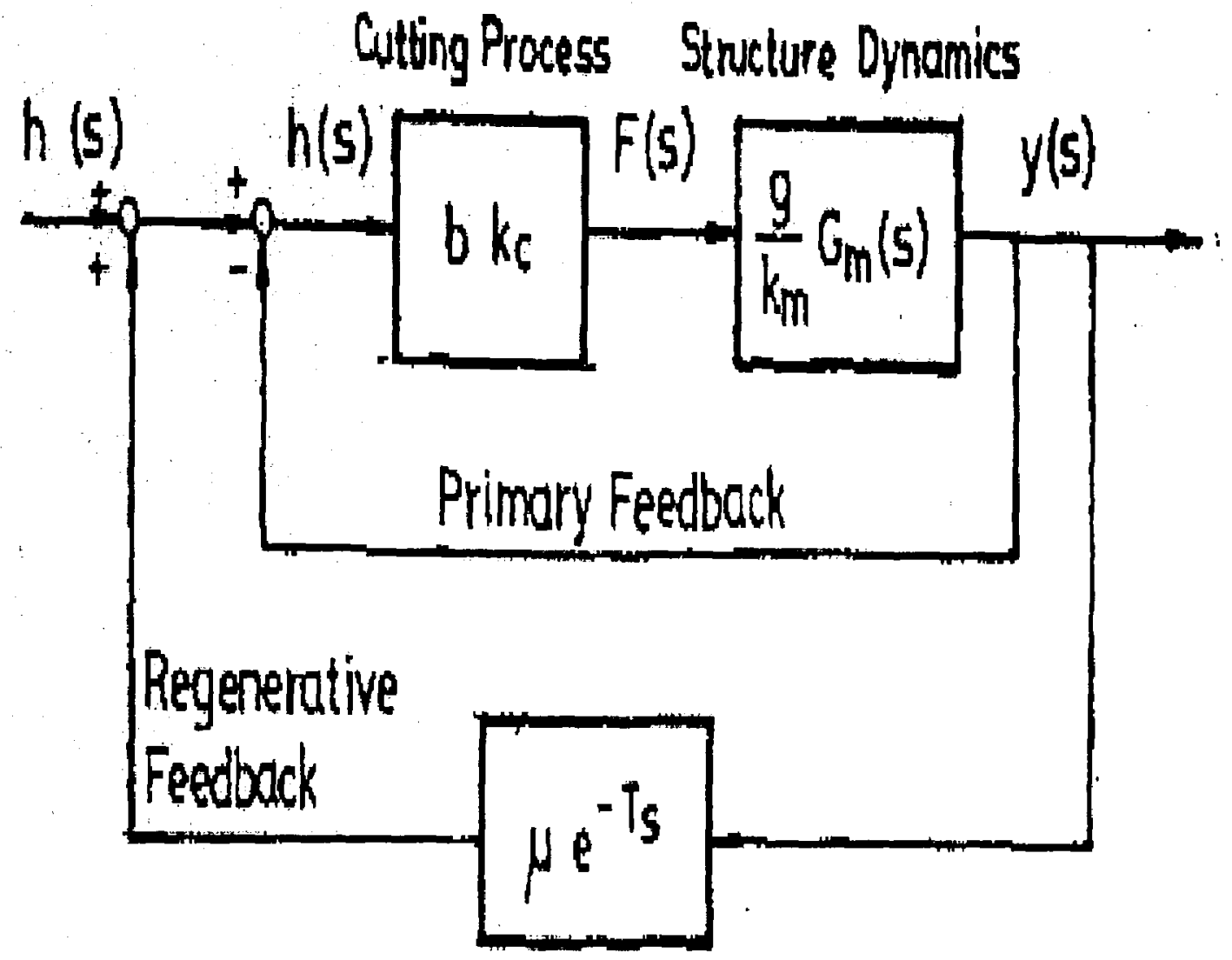

Figure 2.4: Block diagram of the regenerative chatter [9]

Based on the identified chatter frequency, a new spindle speed is computed by applying the principal of keeping the phase between the present and previous undulations to $90^{\circ}$. The new speed command is executed while the cutting proceeds. Figure 2.5 shows simulation results for this method [9]. The main disadvantages of this technique are that changes in cutting speed affect the surface finish of the part.

Simulation results shows that the chatter can be suppressed by using this approach. 


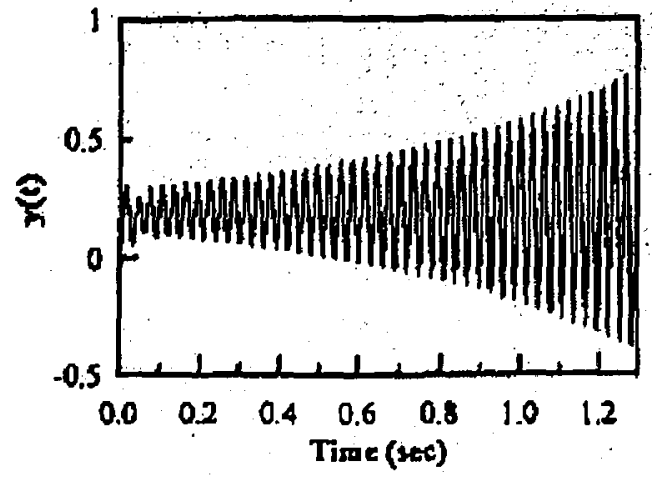

(a)

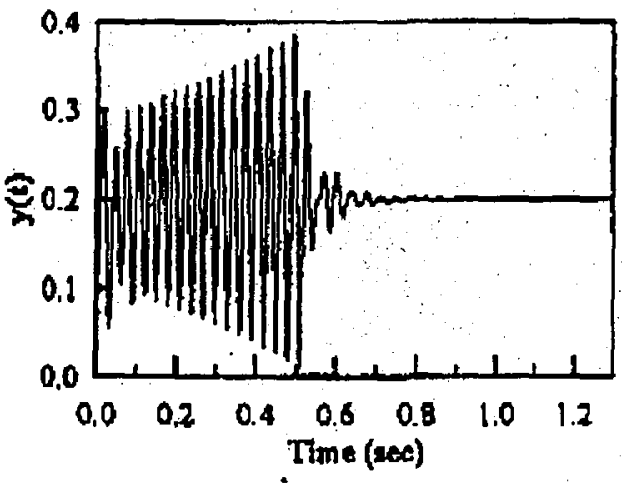

(b)

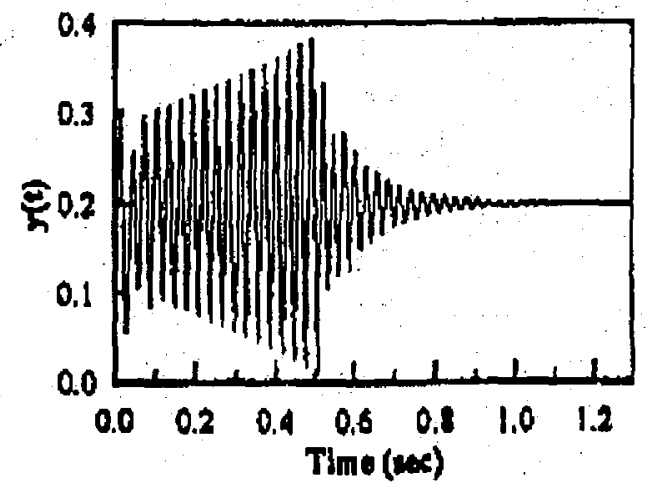

(c)

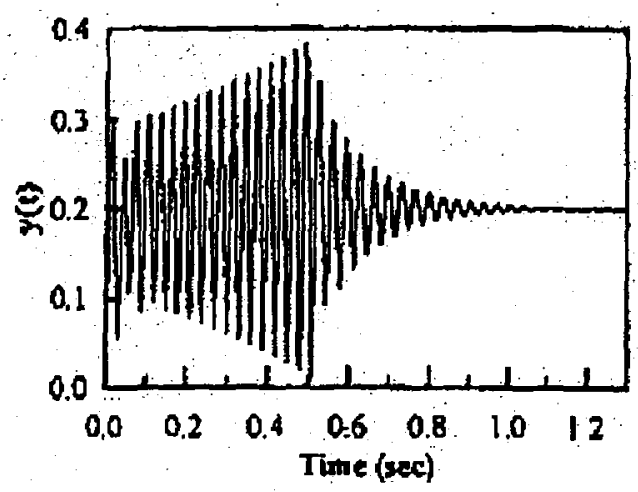

(d)

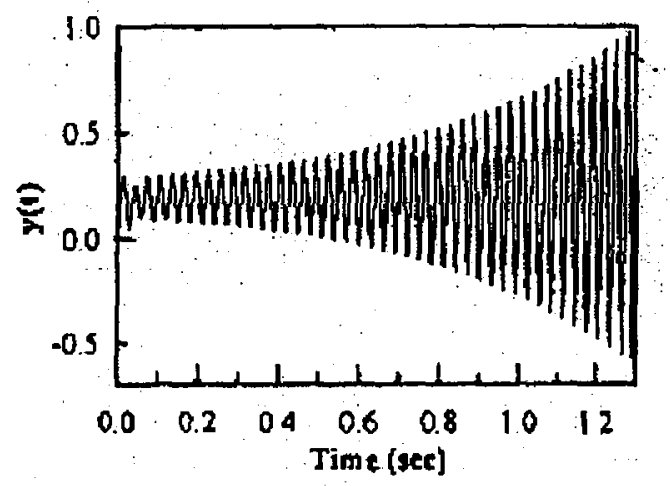

(e)

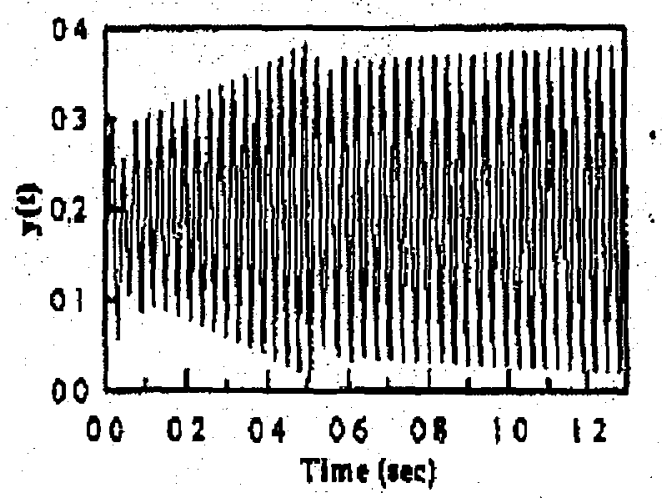

(f)

Figure 2.5: Simulation of the placement $y(t)$ for (a) without control, and under control with (b) $v=0.25$, (c) $v=0.4$, (d) $v=0.1$, (e) $v=0.75$, and (f) $v=1$ [9] 
The online information needed in applying the proposed approach is the chatter frequency. This is different from other approaches, which require a complete and accurate model of the system. The experimental set-up is shown schematically in Figure 2.6.

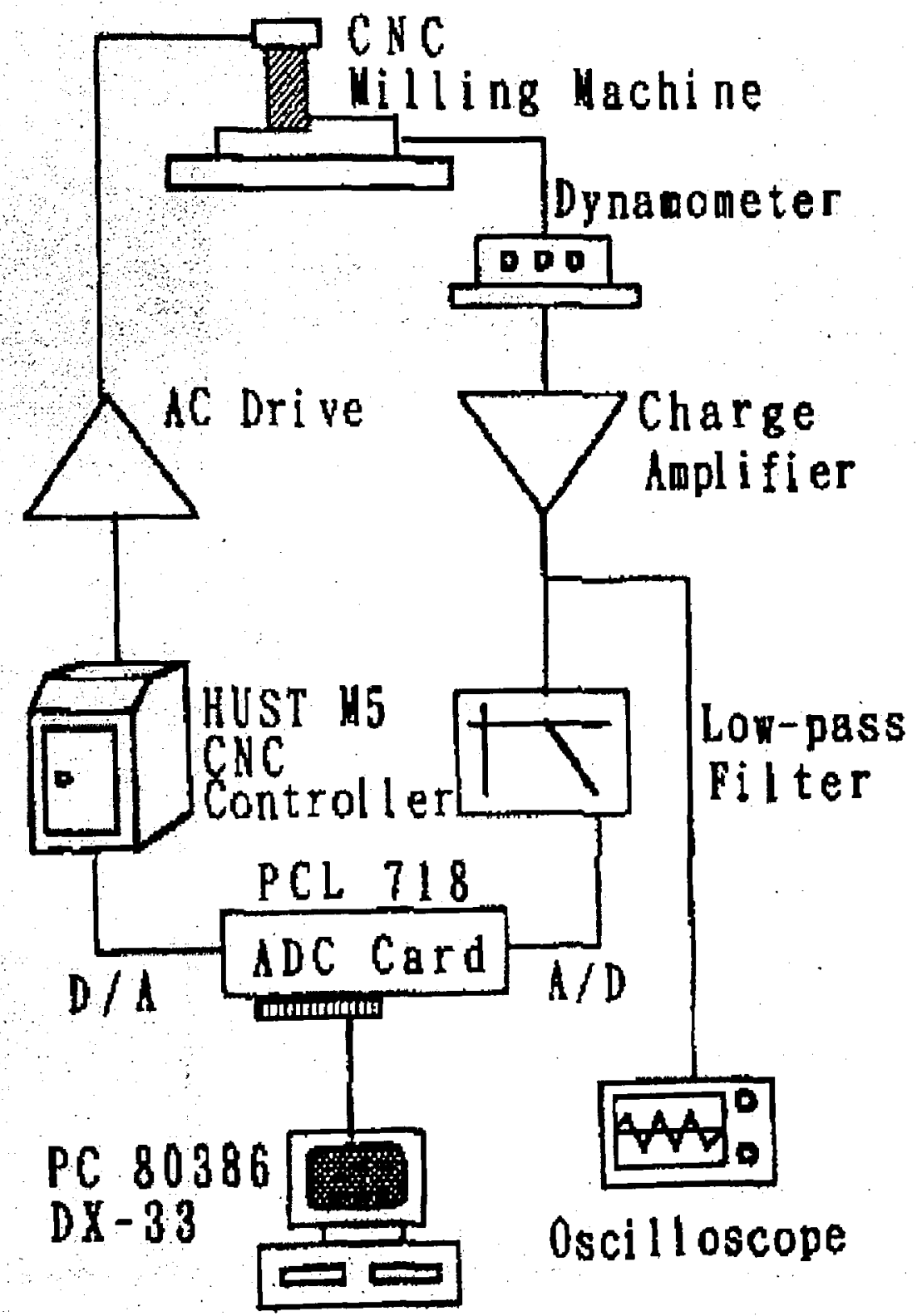

Figure 2.6: Experimental setup [9] 
The tool used is a four-teeth high-speed steel end milling cutter, $12 \mathrm{~mm}$ in diameter. The work materials are S45C and pure aluminum of size $160 \times 140 \times 25 \mathrm{~mm}$, to conform with the size of dynamometer. The initial speed is set too $1000 \mathrm{rpm}$. The speed will reduce when chatter occurs. This is because the machine tool and cutting tool used in the experiment limited the spindle speed to run no more than $1000 \mathrm{rpm}$. By applying the proposed method to other machines, the initial speed can be set to any value within the capacity of the installed motor of the machine tool.

This method is continually varying the spindle speed, while cutting is effective in reducing the amplitude of self-exited chatter and it is applicable when slight chatter can be tolerated, provided that the amplitude never exceeds a given limit.

Spindle speed regulation systems, which simply seek out any stable zone, leave the process susceptible to abrupt changes in the machining system's dynamics.

\subsubsection{Viscoelastic Dynamic Damping}

Kim et al. [11] proposed the procedure for optimum design of a viscoelestic dynamic damper. The machining chatter was effectively suppressed by applying the damper on the tool post. Based on the dynamic properties of a selected viscoelastic material, which are obtained from excitation and response measurements on a simple system having a viscoelastic element, an optimal dynamic damper is designed and attached to the tool post. Its performance is verified by improved cut surface profiles, and the decrease in the magnitude of the transfer functions around the chatter frequency.

The dynamic properties of a viscoelastic material can be experimentally obtained from excitation tests on a single DOF system composed of the viscoelastic element and a lumped mass $M$ at given prestrains and temperatures ( Figure 2.7). 


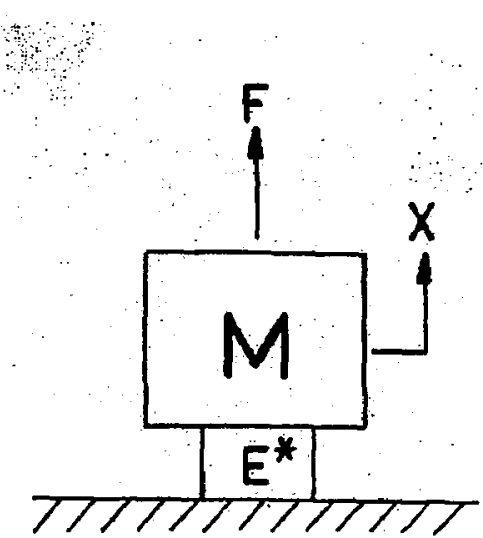

(a) Force Excitation

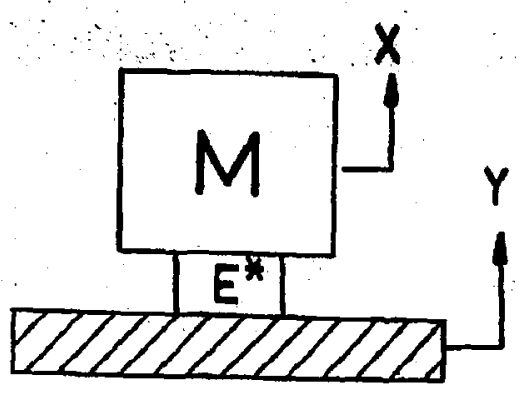

(b) Base Excitation

Figure 2.7: Excitation Methods [11]

The complex elastic modulus of a viscoelestic material is given by

$\mathrm{E}^{*}=\sigma / \mathrm{C}=\mathrm{E}(1+\mathrm{j} \delta)$

$\mathrm{E}=$ Elastic factor, $\delta=$ loss factor is in general dependent upon the oscillating frequency, ambient temperature and applied preload prestrain [11]. Considering hollow type viscoelastic element with inner diameter $d_{i}$ and outer diameter $d_{0}$, and original height $L_{0}$, The apparent complex elastic modulus $\mathrm{E}_{\mathrm{a}}$ can be expressed by

$\mathrm{E}_{\mathrm{a}}=\frac{F / A}{X / L}$

Where, $\mathrm{F}=$ axial load, $\mathrm{A}=$ cross sectional area, $\mathrm{X}=$ deformed length due to $\mathrm{F}, \mathrm{L}=$ height after preload, $E_{s}=$ elastic modulus.

In order to estimate the elastic modulus and loss factor of a given viscoelastic material, a single DOF system was constructed as shown in Figure 2.7 (a) with the viscoelastic element and a lumped mass. The prestrain was adjusted by tuning a nut, which can be rotated on the well-lubricated adjapter. Assuming that the ambient temperature was held constant through the experiments, impulse-response tests were run at several prestrains. Elastic modulus $\mathrm{E}(\omega)$ and loss factor $\delta(\omega)$ at each given prestrains were calculated from 
the measured transfer functions [11].

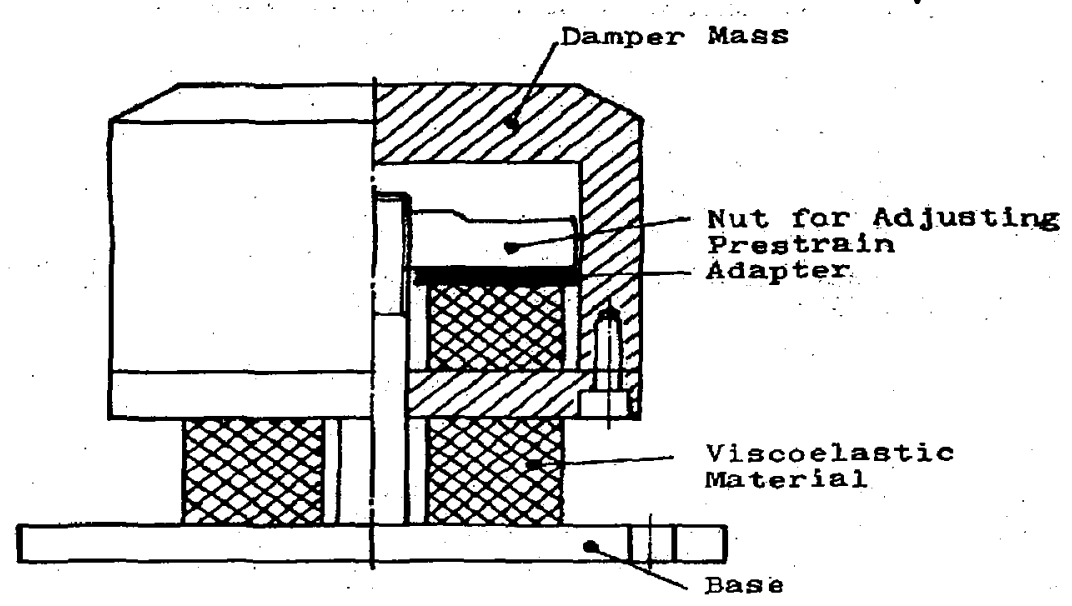

Figure 2.7 (a): Structure of a viscoelastic dynamic damper [11]

For the purpose of identifying the chatter frequency of the lathe, cutting experiments were performed under rather heavy conditions. Experimental results show that the machining chatter was suppressed about $55 \%$ in terms of integrated power of the vibration signal around the chatter frequency [11]. This was achieved by attaching the optimized viscoelastic damper to the tool post at a fixed location as shown in Figure 2.8.

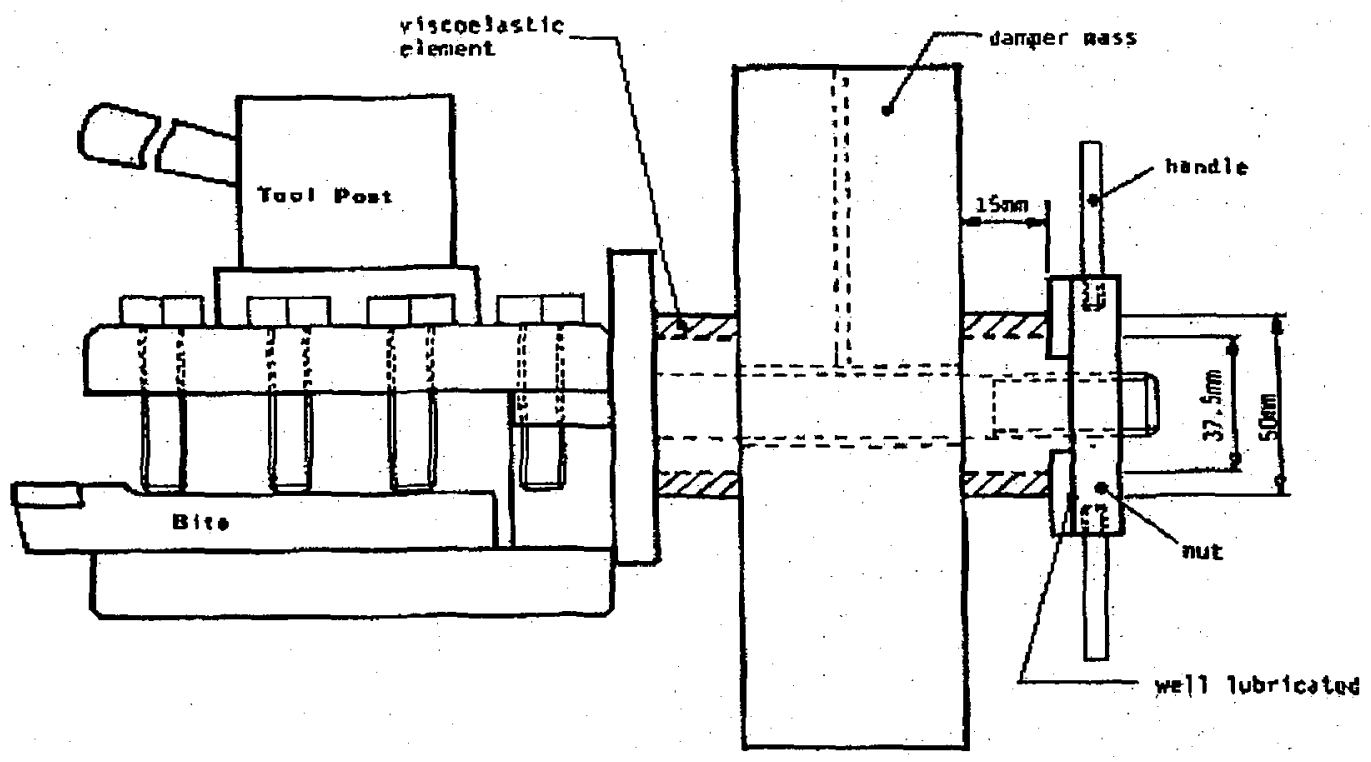

Figure 2.8 Installation of the dynamic damper on the tool post and its dimensions [11] 


\subsubsection{Vibration Cutting}

Xiao et al. [12], showed through experimental investigation that chatter is effectively suppressed without relying on the tool geometry, and work displacement amplitudes are reduced from a wide range of $10-12 \mu \mathrm{m}$ to the range of $3-5 \mu \mathrm{m}$ by applying vibration cutting, a new cutting model which contains a vibration cutting process is proposed. The vibration cutting setup is shown in Figure 2.9

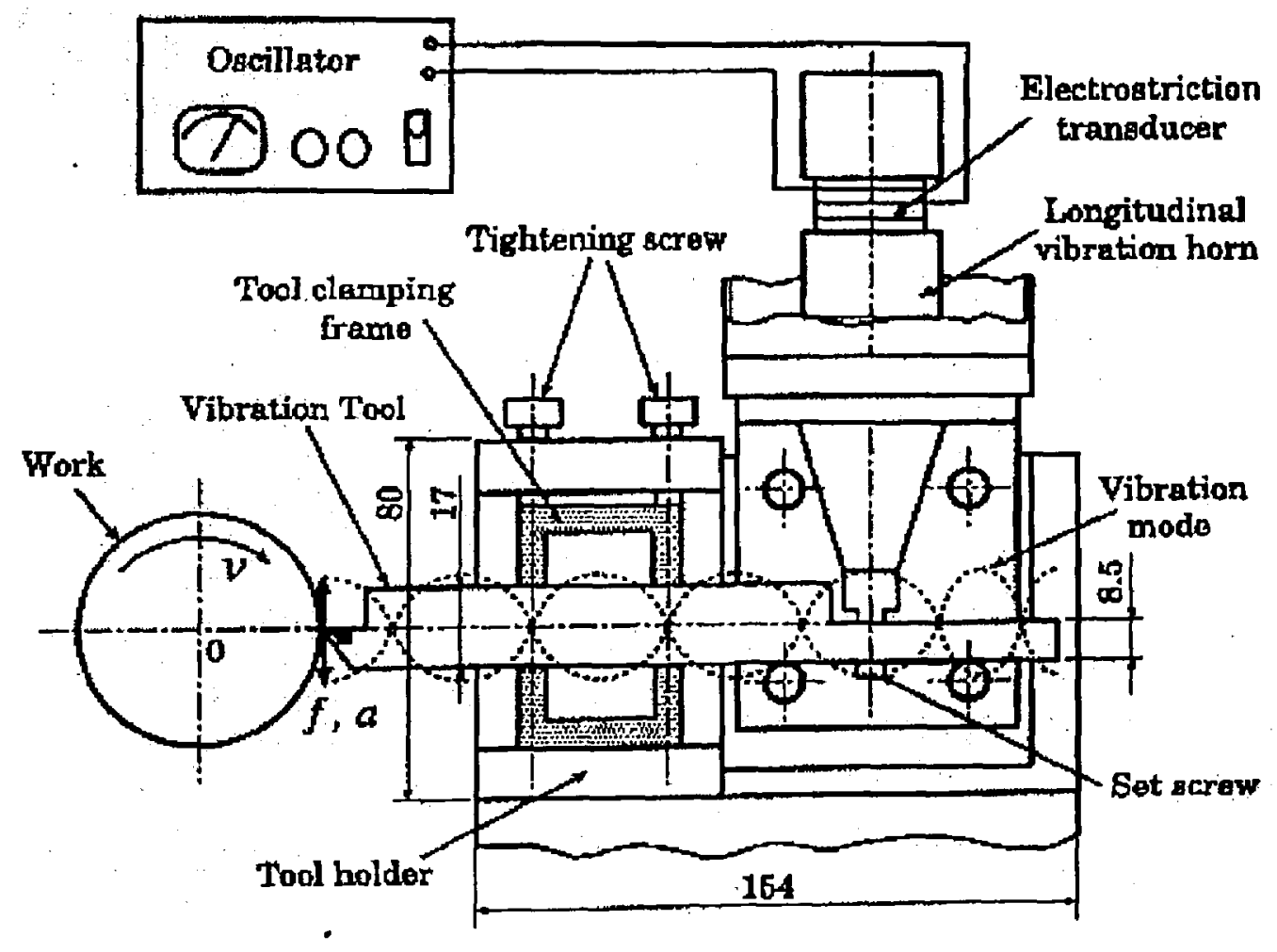

Figure 2.9: Vibration Cutting Setup [12]

The setup consists of a rotary work piece with a cutting speed $v$ and a vibrated tool employed by an ultrasonic electrostriction transducer. When the frequency and the amplitude become equal to zero, the cutting setup is a conventional cutting. An eddy 
current sensor with its frequency response ranging from $100 \mathrm{~Hz}$ to $80 \mathrm{kHz}$ is set to measure the work displacement in the y direction as shown in Figure 2.10.

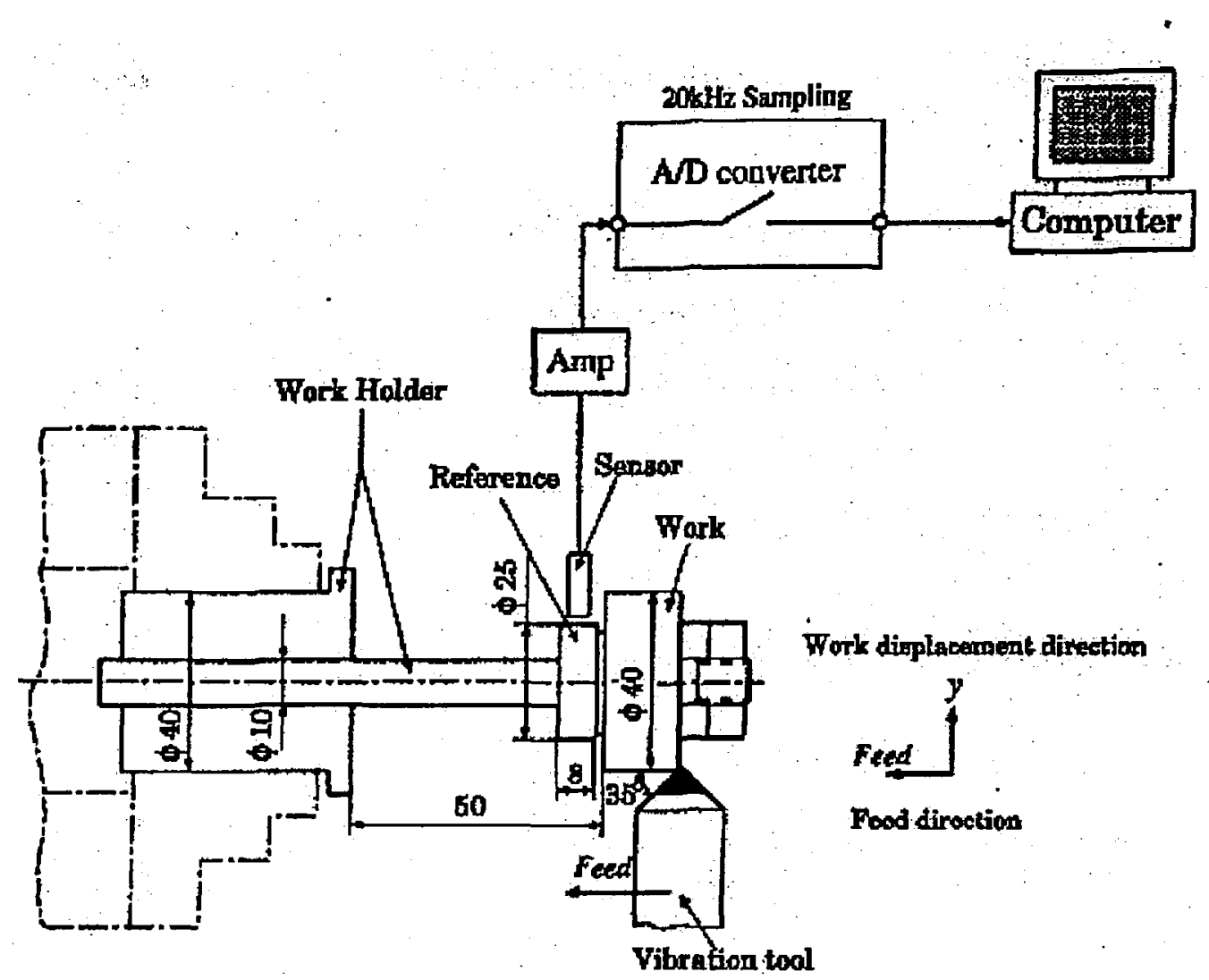

Figure 2.10: Work Holder and Measurement Setup for Work Displacement [12]

The analog signal provided by the sensors is converted to digital form at a sampling frequency of $20 \mathrm{kHz}$ and then stored in a computer. The work piece, supported by a slender holder, is S45C carbon steel. The rotary speed is set to be $460 \mathrm{rpm}$ so that an approximate cutting speed of $58 \mathrm{~m} / \mathrm{min}$ is achieved.

In order to observe the effect of chatter suppression during vibration cutting, four different tool geometries were used. The side cutting was chosen to be $35^{\circ}$ and the corner radius of the tool was approximately $0.05 \mathrm{~mm}$. 

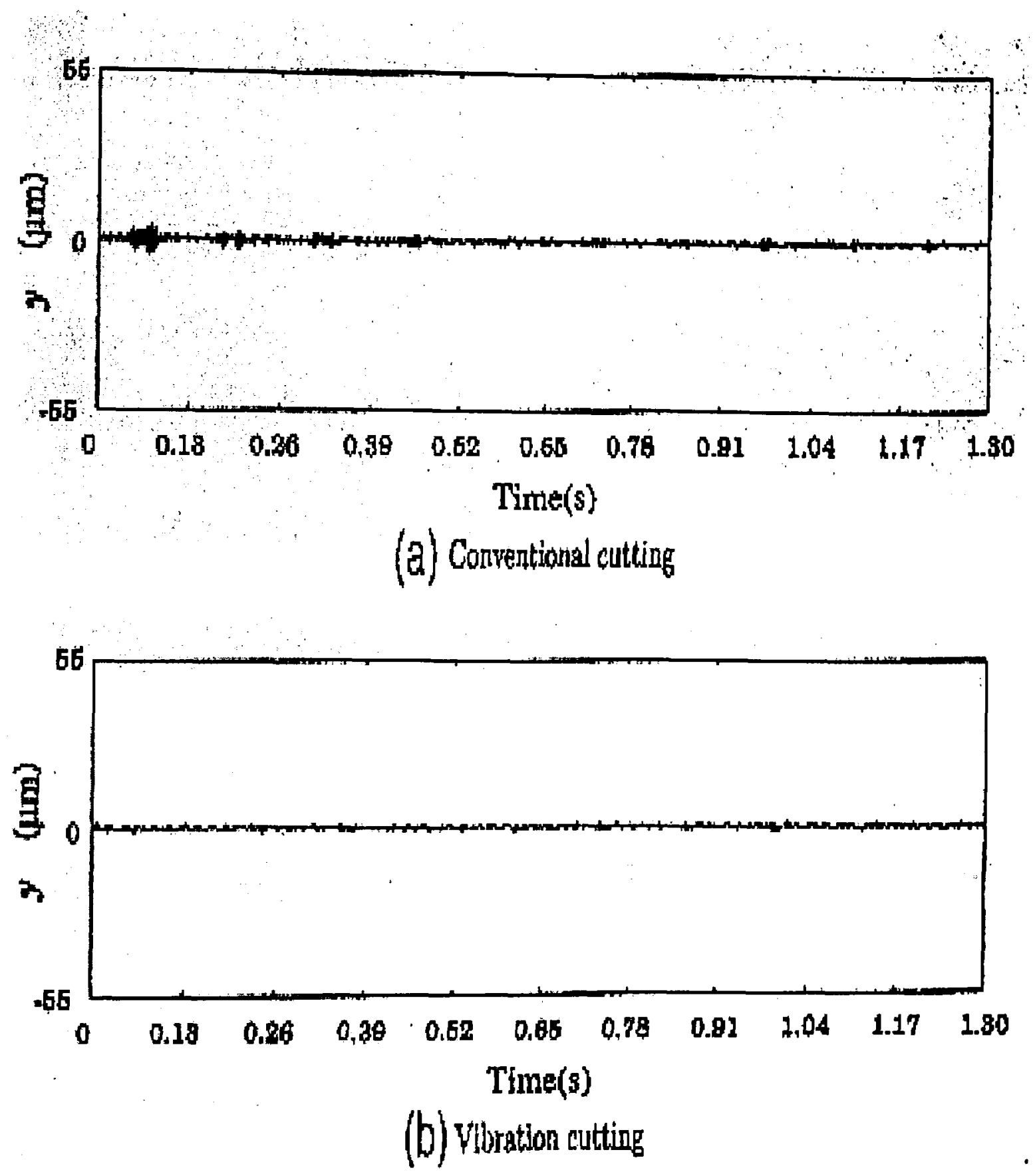

Figure 2.11: Experimental work displacement with tool rake angle $\alpha_{0}=9^{0}$ and tool Clearance angle $\gamma_{0}=1^{0}[12]$

Figures 2.11 (a), 2.12 (a), 2.13 (a), 2.14 (a) shows the results with four different tool geometries obtained by conventional cutting. In Figures 2.11 (a) and 2.12(a), the work displacement amplitude ranges from 10 to $14 \mu \mathrm{m}$ and is kept in a favorable cutting state. 
The results show that a relatively high cutting stability can be obtained by using large rake angle and small clearance angle.

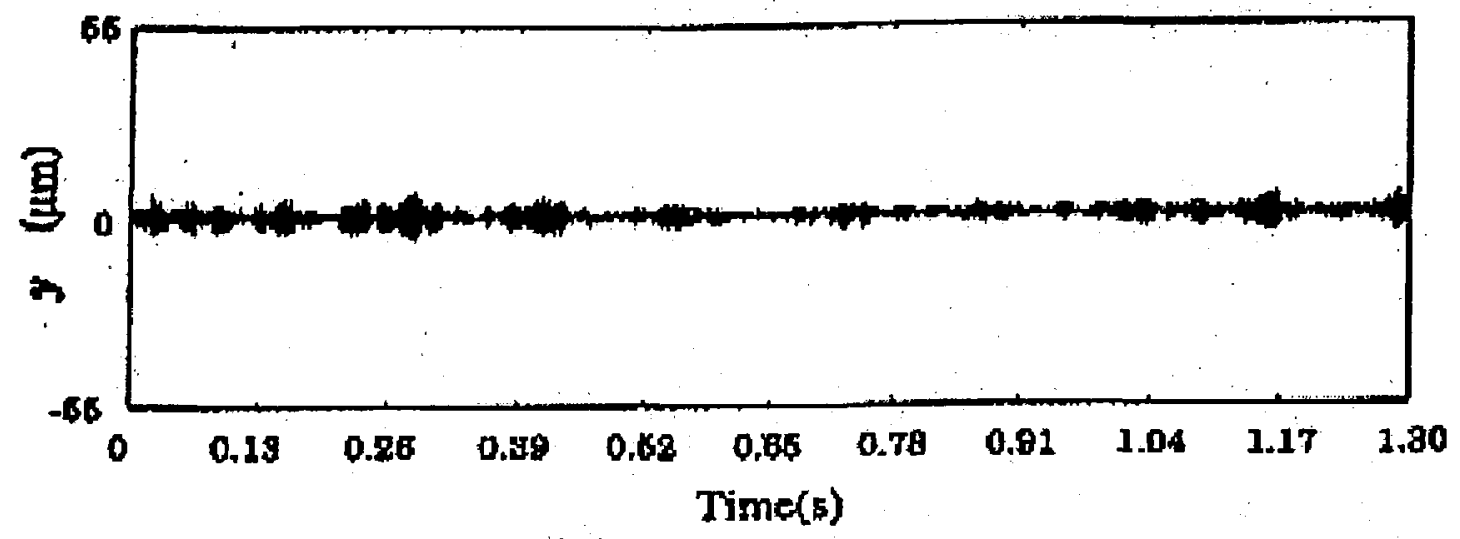

(a) Conventional cutting

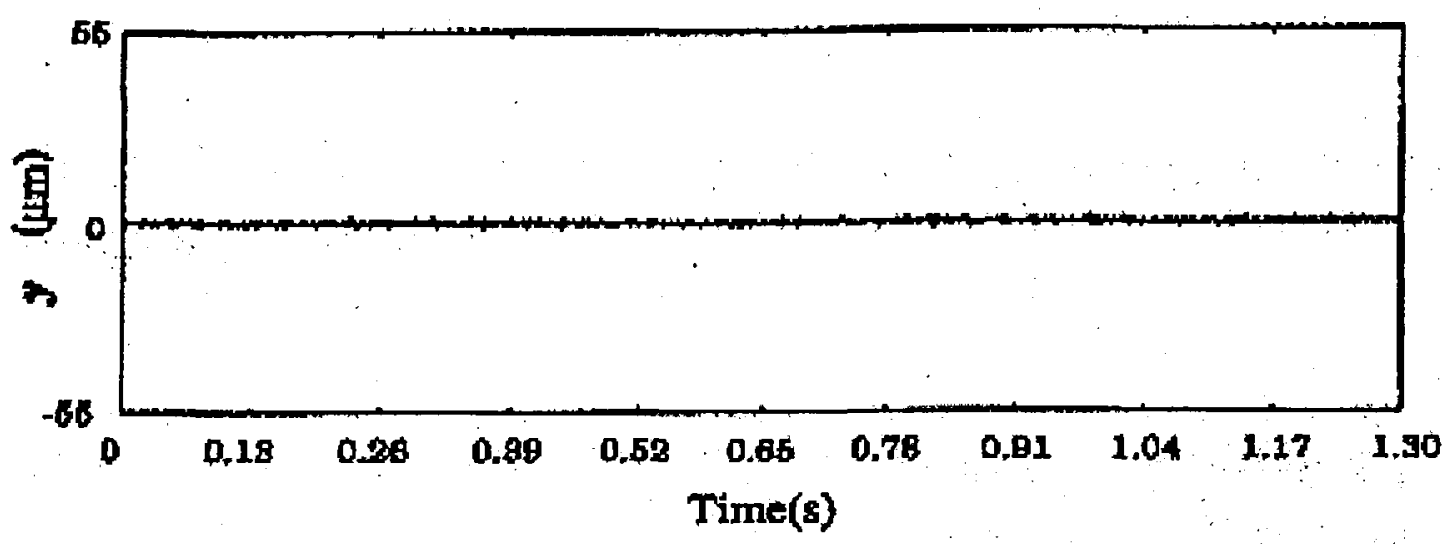

(b) Vibration cutting

Figure 2.12: Experimental work displacement with tool rake angle $\alpha_{0}=6^{0}$ and tool Clearance angle $\gamma_{0}=4^{0}[12]$

In figure 2.13 (a) and 2.14 (a), the maximum amplitude values of the work displacement are 55 and $102 \mu \mathrm{m}$, respectively. The results show that chatter is caused by the decrease in rake angle and an increase in clearance angle. 

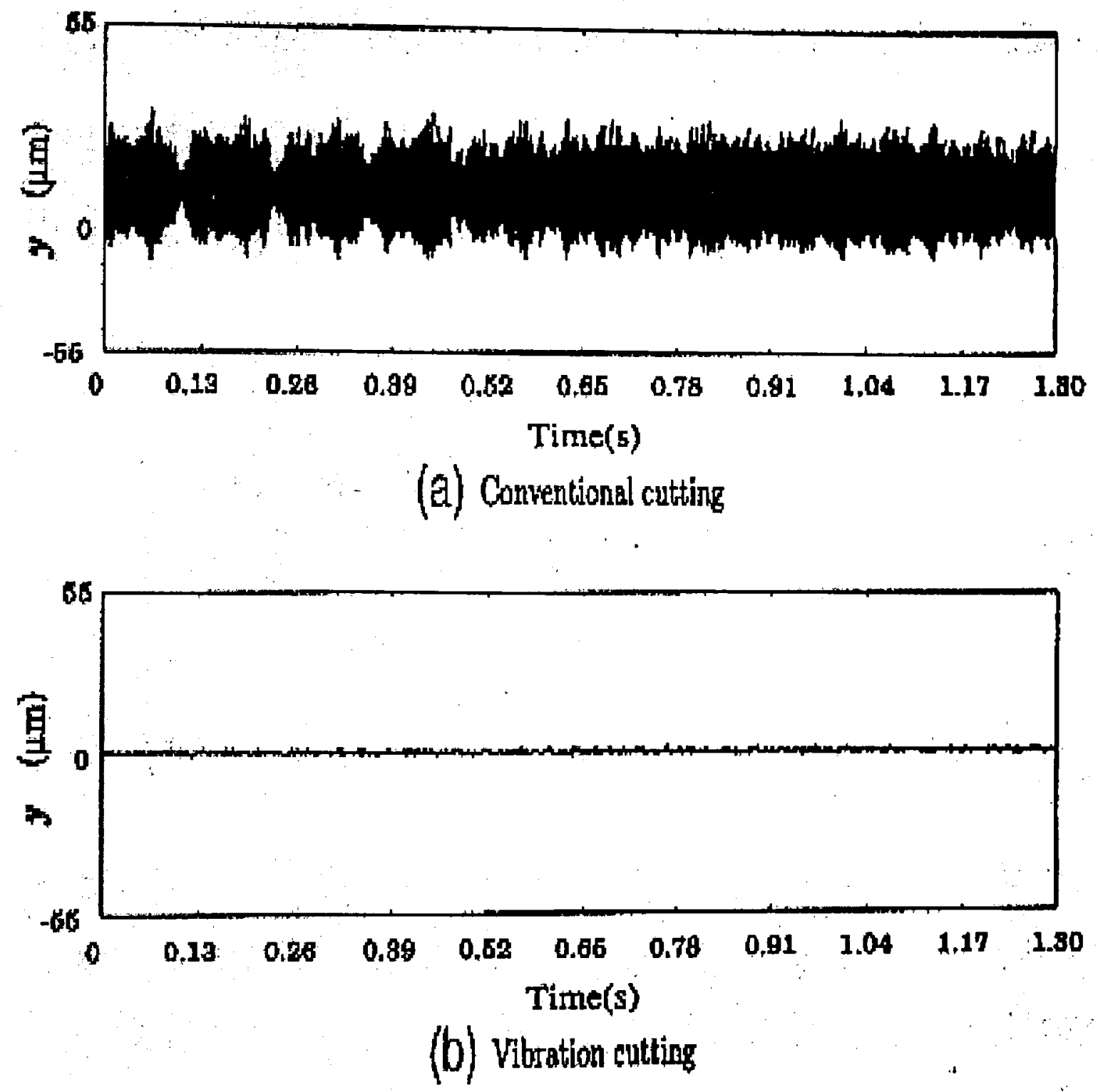

Figure 2.13: Experimental work displacement with tool rake angle $\alpha_{0}=3^{0}$ and tool Clearance angle $\gamma_{0}=7^{0}$ [12] 


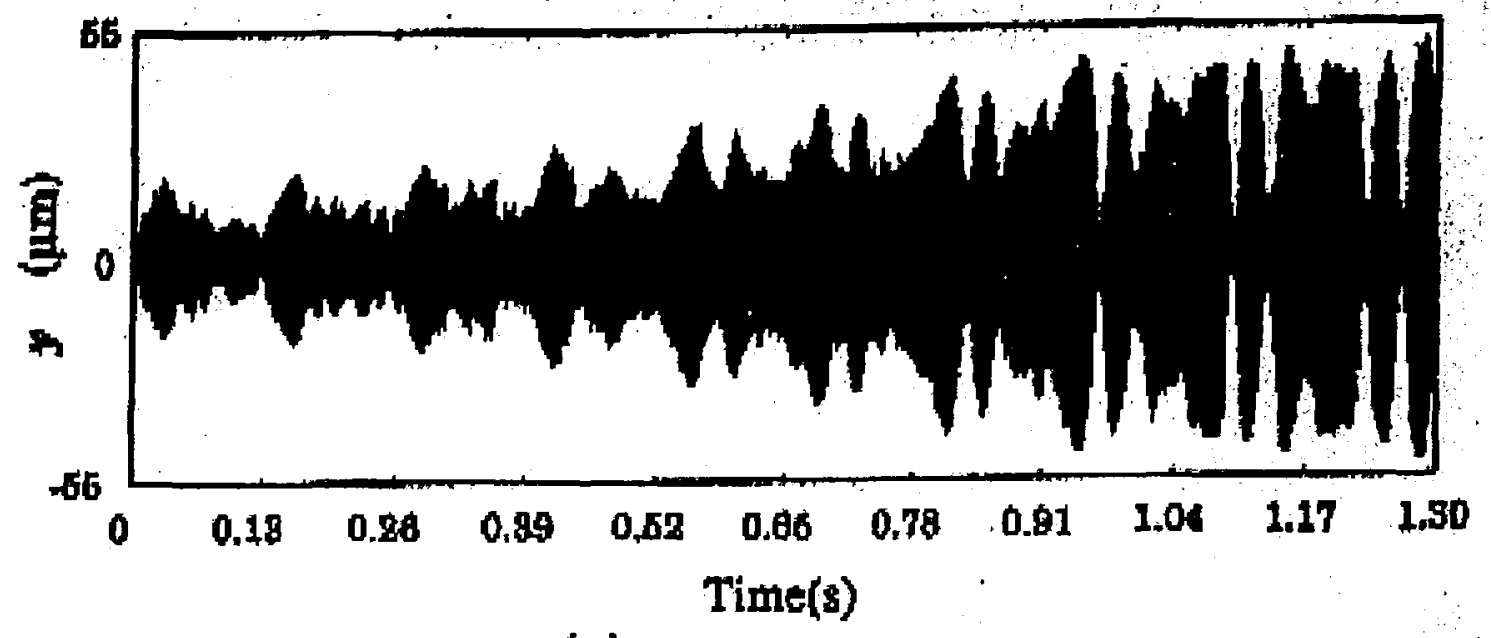

(a) Conyentional culting

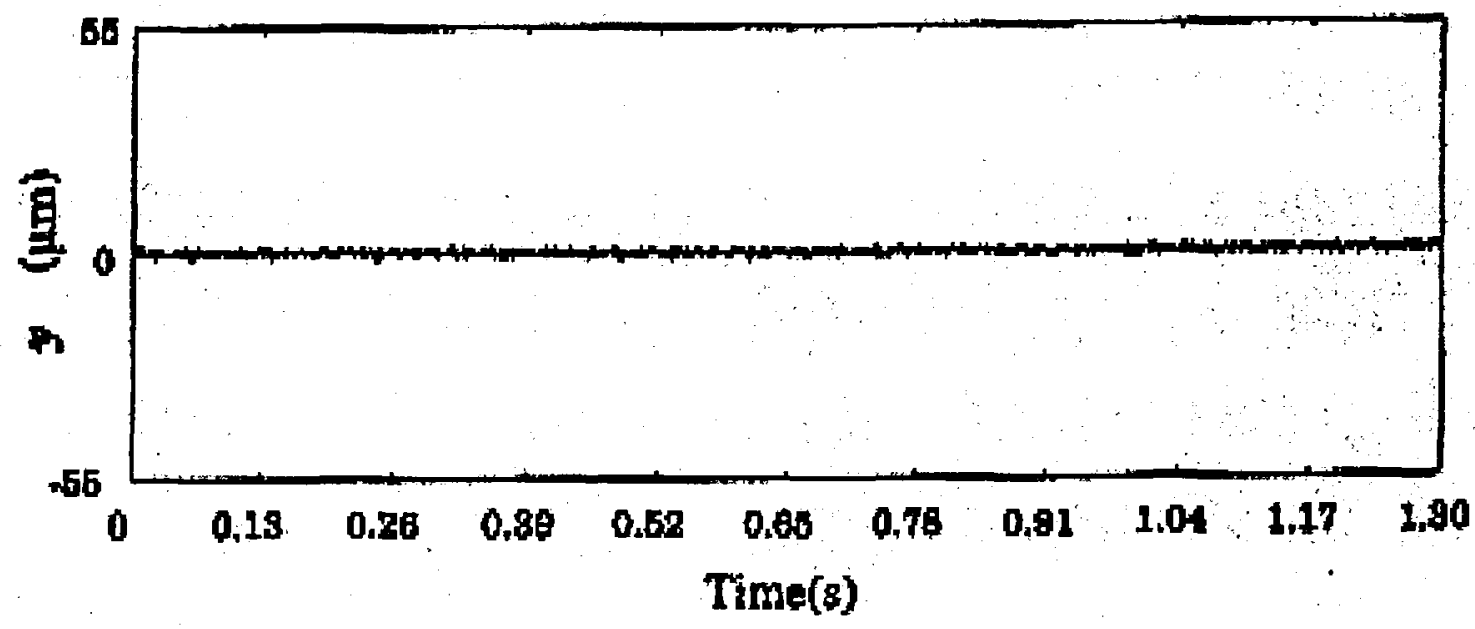

(b) Vitiration cutting

Figure 2.14: Experimental work displacement with tool rake angle $\alpha_{0}=0^{0}$ and tool Clearance angle $\gamma_{0}=10^{0}[12]$

Figures 2.11(b), 2.12 (b), 2.13 (b), and 2.14 (b) show the results with four different tool geometries obtained by vibration cutting. The work displacement amplitudes are 3.3, 3.9, 4.2 , and $4.5 \mu \mathrm{m}$, respectively, and each work oscillation is kept in a stable state. 


\subsubsection{Vibration Absorber}

Tarng et al. [13] focused on developing a simple chatter suppression method based on the improvement of the dynamic response of a cutting tool with a tuned vibration absorber. Basically, the use of the tuned vibration absorber for the suppression of chatter can be considered a passive control method. A piezoelectric inertia actuator is mounted on the cutting tool and acts as a tuned vibration absorber for the suppression of chatter in turning operations

The tuned vibration absorber can modify the frequency response function of the cutting tool so as to improve cutting stability in cutting operations. The stability limit of the cut width is inversely proportional to the magnitude of the real part of the frequency response function of the machine tool structure. Therefore the suppression of chatter is feasible if the magnitude of the real part of the frequency response function of the machine tool structure is reduced (Figure 2.15). A piezoelectric inertia actuator mounted on the cutting tool is used as to tune vibration absorber for reducing the magnitude of the real part of the frequency response function of the cutting tool in turning operations. This is because the

tuned vibration absorber introduces a new degree of freedom to the dynamic system of the cutting tool. The new frequency response function of the cutting tool due to the tuned vibration absorber mounted on the cutting tool can then be obtained. The absorber with damping, when tuned to the proper frequency, can greatly reduce the magnitude of the negative real part of the frequency response function of the cutting tool so as to improve cutting stability.

The cut width $b$ at the stability limit is called the critical cut width $\mathrm{b}_{\text {lim, rr }}[5]$, which can be expressed as:

$$
\mathbf{b}_{\mathrm{lim}, c r}=\frac{-1}{\left.2 K s \operatorname{Re}(G)_{\min }(j \omega)\right) n e g}
$$

$\operatorname{Re}(\mathrm{Gm}(j \omega))_{\text {neg }}$ is the real part of the frequency response function of the cutting tool and $\omega$ the chatter frequency in $\mathrm{rad} / \mathrm{s}$. 


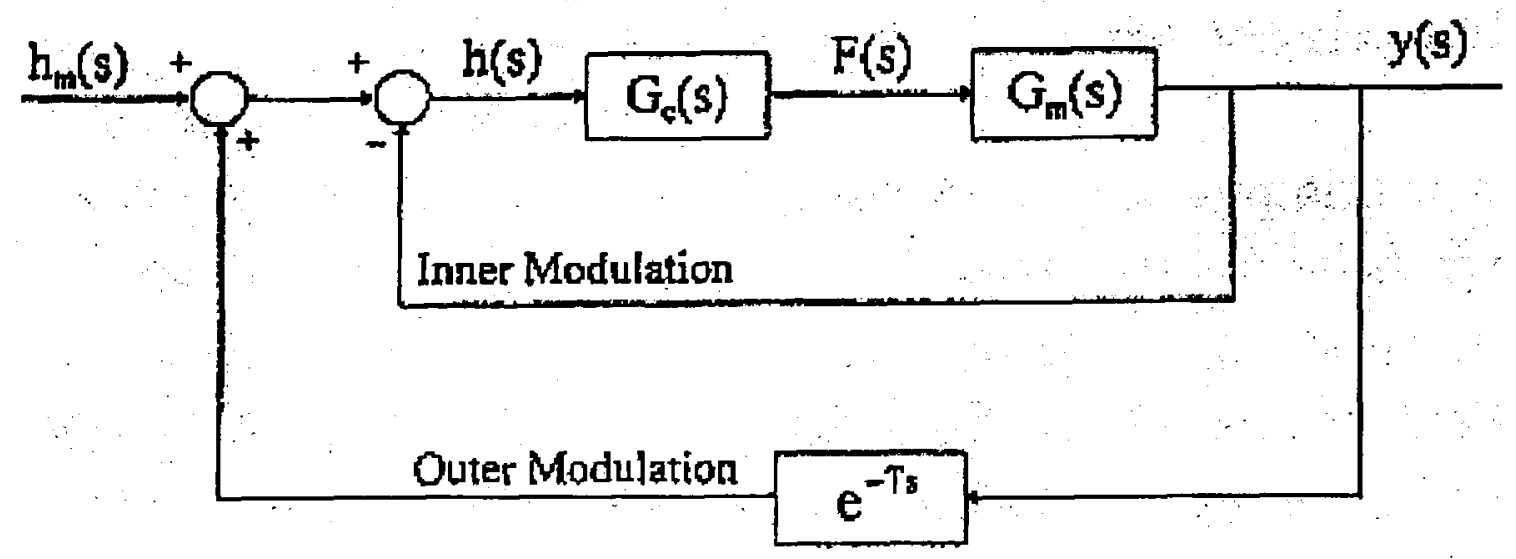

Figure 2.15: Control block diagram for chatter in turning [13]

The following Figure 2.16 shows the real part and magnitude of the frequency response function of the cutting tool.
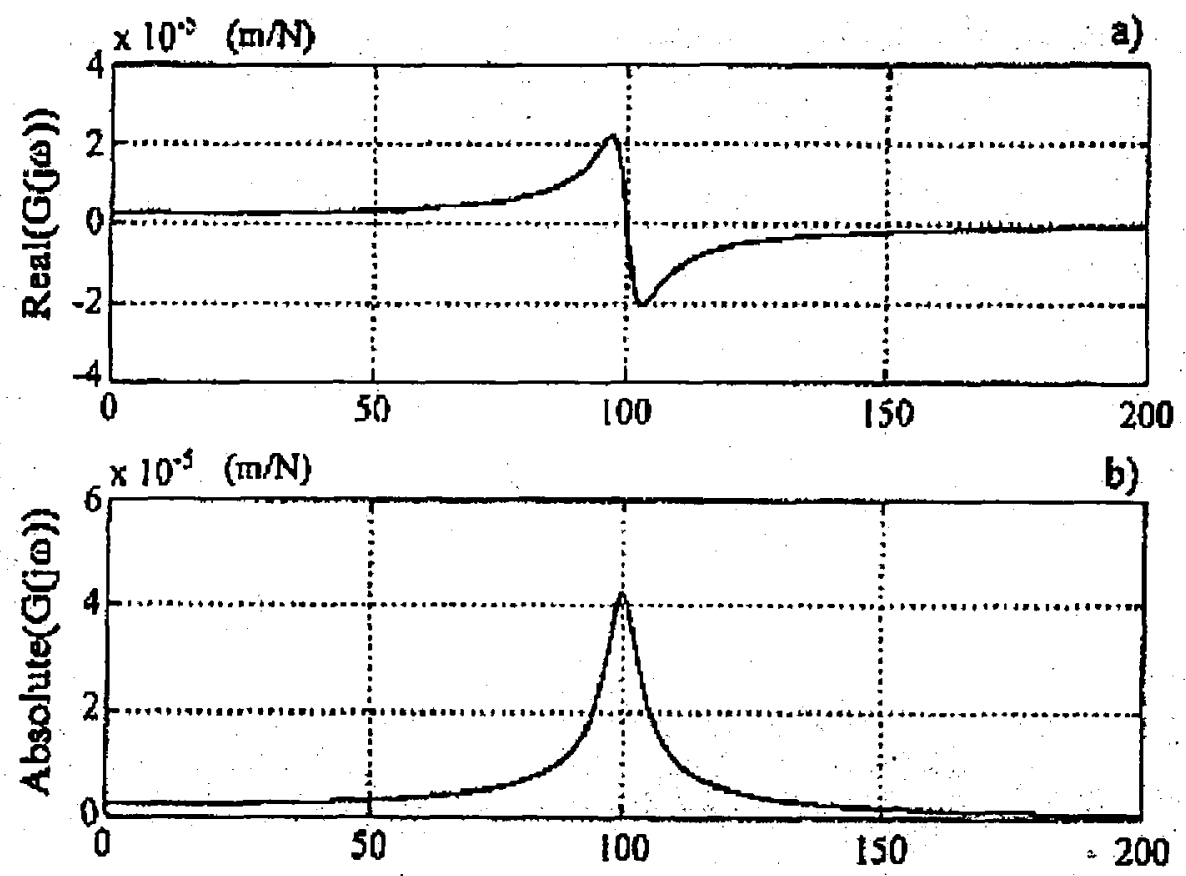

Figure 2.16: A simplified response function of the cutting tool: (a) real part; (b) magnitude [13] 
Figure 2.17 shows the chatter frequency and the critical cut width $\mathrm{b}_{\lim }$ as a function of spindle speed. Chatter will occur when the cut width $b$ is greater than $b_{\text {lim. }}$. However, the critical cut width $b_{\text {lim }}$ can be increased if the magnitude of the real part of the frequency response function of the cutting tool is reduced.
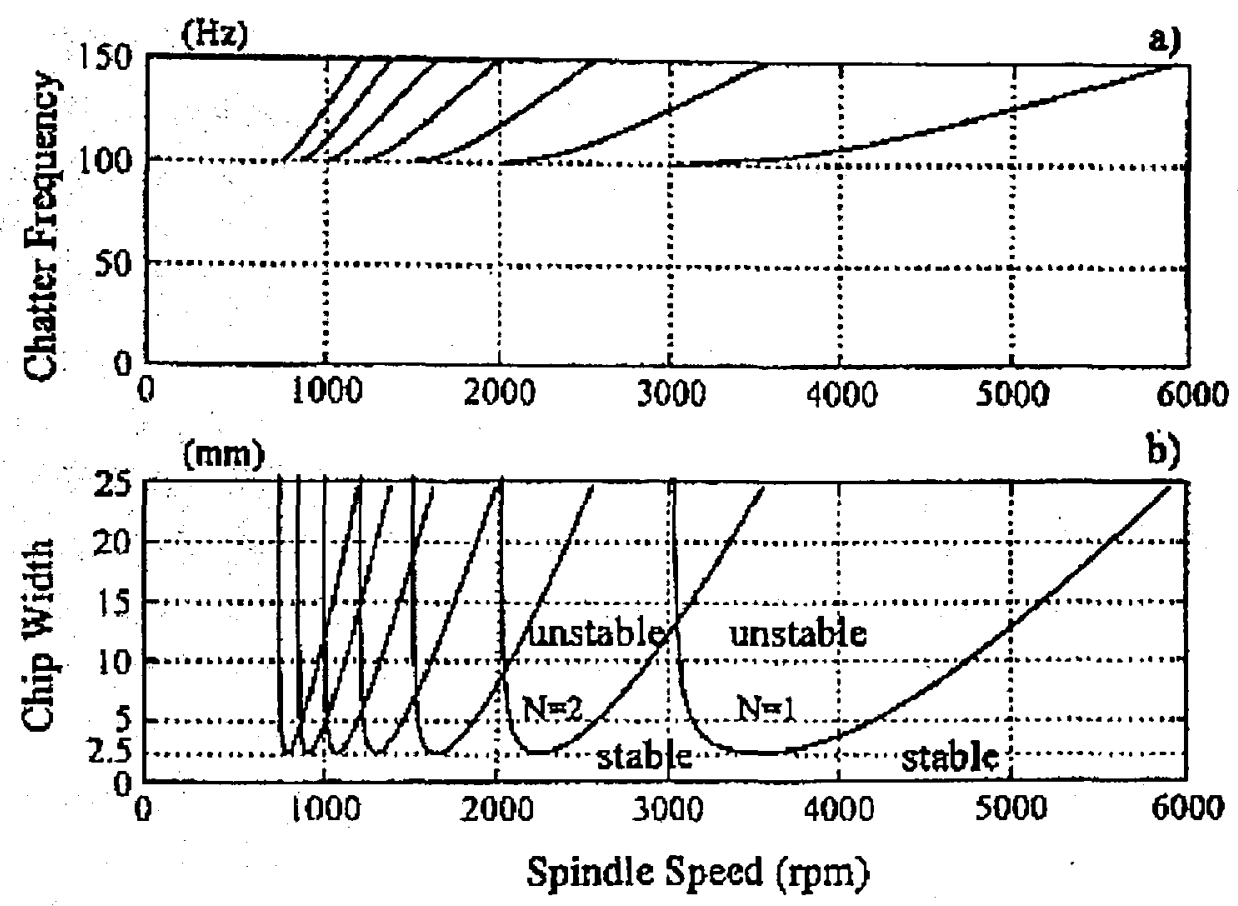

Figure 2.17: Simulated stability chart : (a) chatter frequency versus spindle speed (b) cut width versus spindle speed [13]

A tuned vibration absorber for the suppression of chatter is a useful method due to low cost, easy implementation and no need for external energy. But it suffers from a major draw back of being ineffective at low frequencies and the need for tuning as the machining dynamics change.

\subsubsection{Viscous Damper}

Slocum et al. [14] proposed the concept of the replicated internal viscous damper in the interior core of the cutting tool to increase damping. This type of damper uses internal beams covered with a viscous material that are then replicated in place inside structural 
members. This viscous fluid first acts as a mold release and then as a very thin viscous shear layer. This type of damper uses regions of a beam that are often left empty for weight reduction as shown in figure 2.18 . The damping beam's neutral axis is displaced from the neutral axes of the structural beam. The damping beam's surface is coated with a viscous fluid that acts as a mold release until a replicated material hardens and takes the shape of the damping beam. Because the neutral axes are not coincident, as the structural beam bends there will relative shear between it and the damping beam. This shear will act across the thin layer of viscous fluid, and energy will be dissipated.

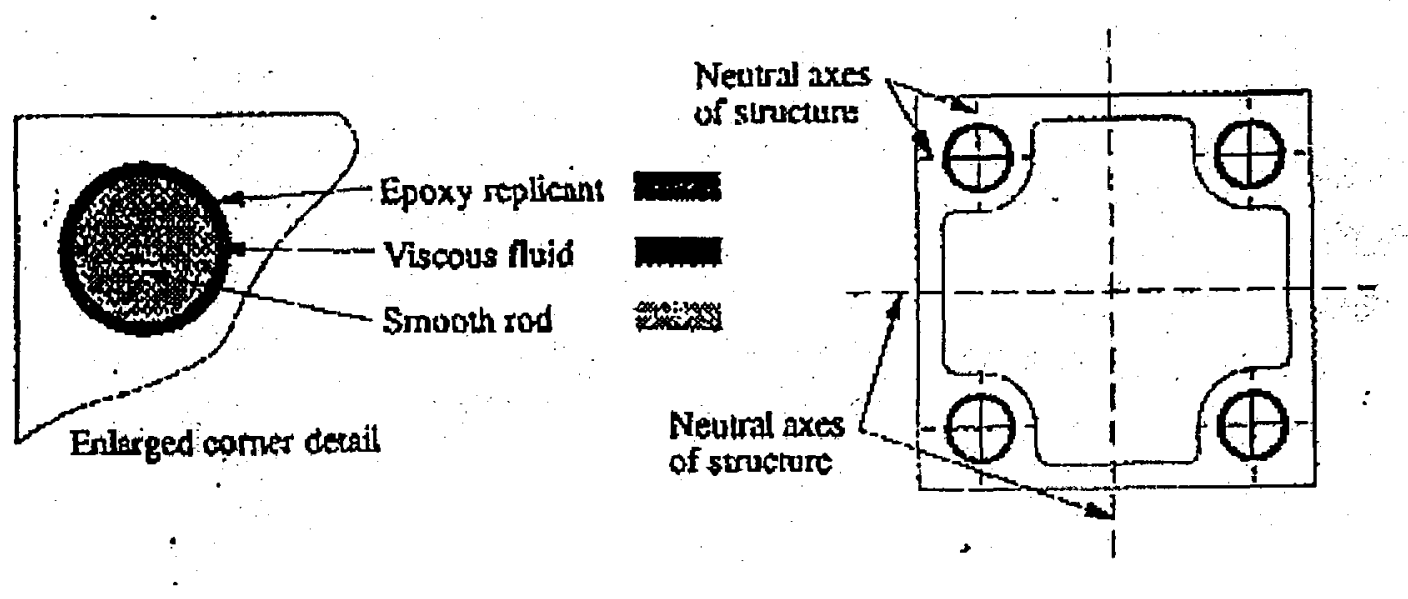

Figure 2.18: Configuration of a beam with replicated internal viscous dampers[14]

The amount of damping that can be achieved with the replicated internal viscous damper can be estimated by calculating the amount of work lost to damping and the total work input to the system. Theory shows that damping can be optimized by maximizing the product of the circumference of the damping member and the axis of the structure.

The replicated internal viscous damper has some advantages: it is easily incorporated into many types of structures and it is inexpensive to fabricate. Viscous shear dampers have the ability to damp out vibrations at all frequencies and they are insensitive to the vibration amplitude. Viscous dampers are often applied to reduce the effects of local excitation sources. For example, these damping devices have been implemented in precision surface grinding applications to vary the dynamic characteristics of the machine 
and suppress unwanted vibrations of the wheel head. However, as higher speeds and greater accuracy are sought, even better means to damp vibration will be required.

\subsubsection{Actuator with Inertia Mass}

Jang et al. [15] used piezoelectric actuators where an inertia mass is attached to a cutting tool and acts as an active vibration damper for suppression of undesired vibration on the cutting tool. The actuator can be well tuned over frequency range by adjusting the size of the inertia mass, so that it can provide a large damping force to suppress undesired vibration of the cutting tool at the resonance frequency of the actuator. The piezoelectric is made up of a stack of disks made of the piezoelectric materials, sealed in titanium housing. The actuator requires an excitation voltage to produce a mechanical displacement or force output due to converse piezoelectric effect. The frequency response function between the input voltage and the output force is shown in Figure 2.19.

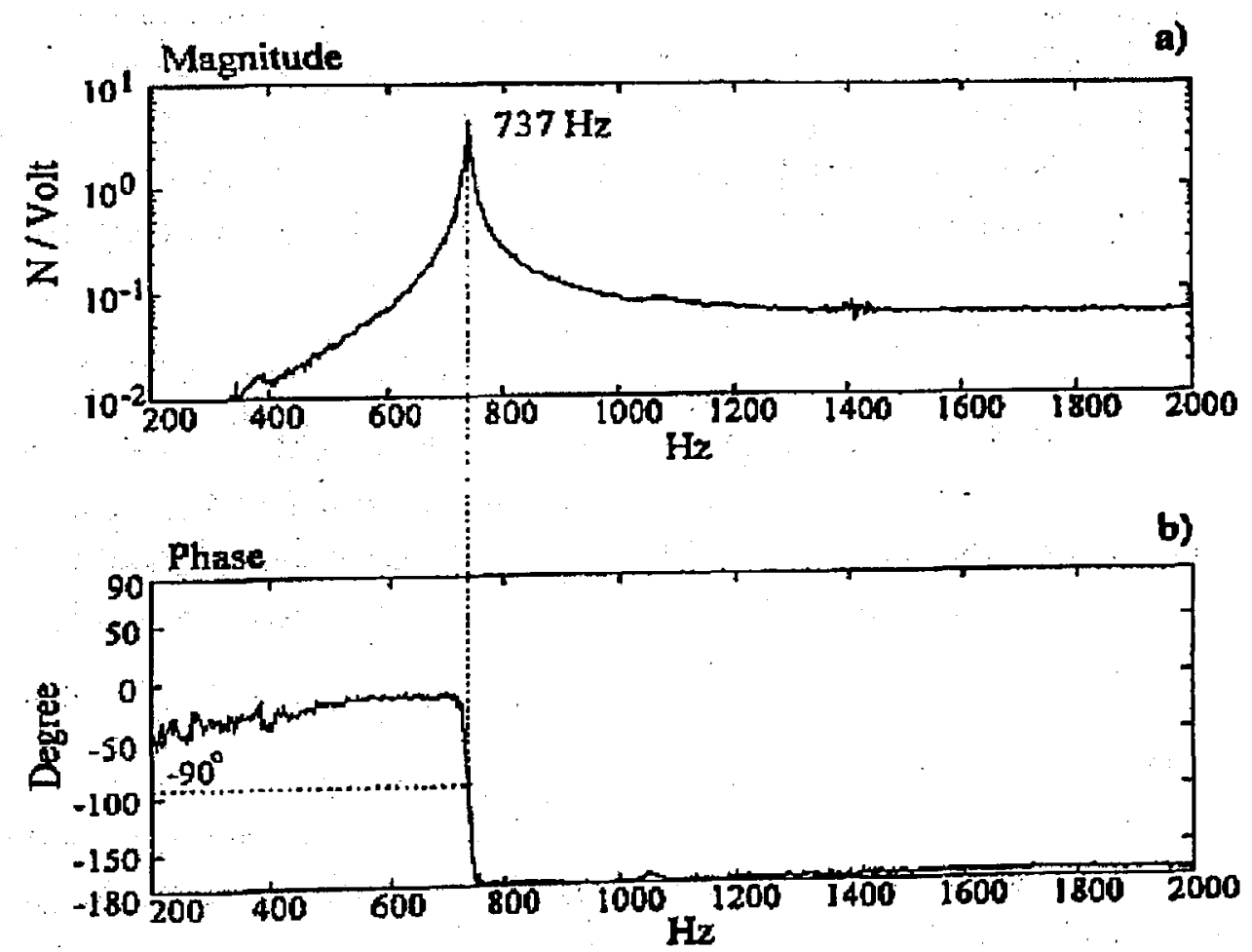

Figure 2.19: Frequency response function between the input voltage and output force of the piezoelectric actuator with a an inertial mass of $150 \mathrm{~g}(\mathrm{a})$ magnitude; (b) Phase [15] 
Figure 2.20 shows a simplified model for the cutting tool with the piezoelectric actuator. The objective is to remove the effect of an external harmonic force $f(t)$ on the cutting tool using the piezoelectric actuator.

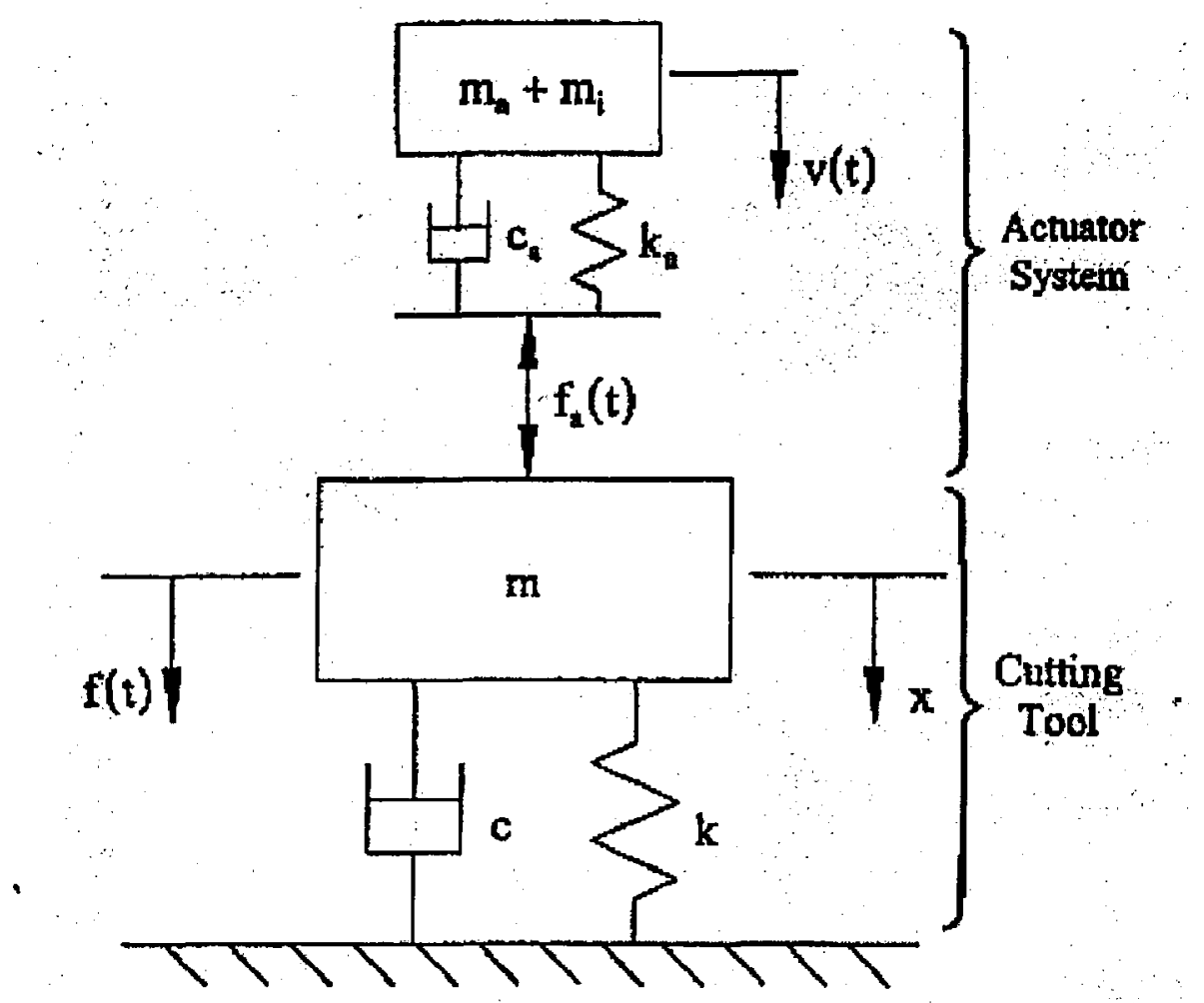

Figure 2.20: A simplified model for the cutting tool with the piezoelectric actuator [15]

Experimental results show that when the cutting tool is excited by a harmonic disturbance the force vibration on the cutting tool can be suppressed upto $90 \%$ with the aid of the piezoelectric actuator.

Use of piezoelectric actuator with an inertial mass is a useful method for suppression of undesired vibration of the cutting tool. The main drawback is the need to change the mass in order to change the frequency response. 


\subsection{Statement of Objective of the Thesis}

The literature review presented in this chapter showed that most active chatter suppression systems need some degree of tuning when confronted with changing dynamics of the machining process.

The objective of this thesis is to develop an active, on-line suppression of chatter in machining. The system will ideally be able to adapt to changing dynamics caused by machine wear and changes in tool and work piece material. 


\section{Chapter 3}

\section{Methodology}

\subsection{Introduction}

The literature review in Chapter 2 discussed different methods that can be used for suppression of chatter. As shown, a generalized method capable of monitoring and controlling non-linear relationship and time delays inherent in the system does not exist. In this chapter, a general method for active suppression of chatter is introduced. The methodology relies on an adaptive, differentiable model of the machining process.

\subsection{Methodology}

The active chatter suppression system presented in this thesis is comprised of an active vibration source with continually adjustable parameters. The idea of active chatter suppression is shown in Figure 3.1.
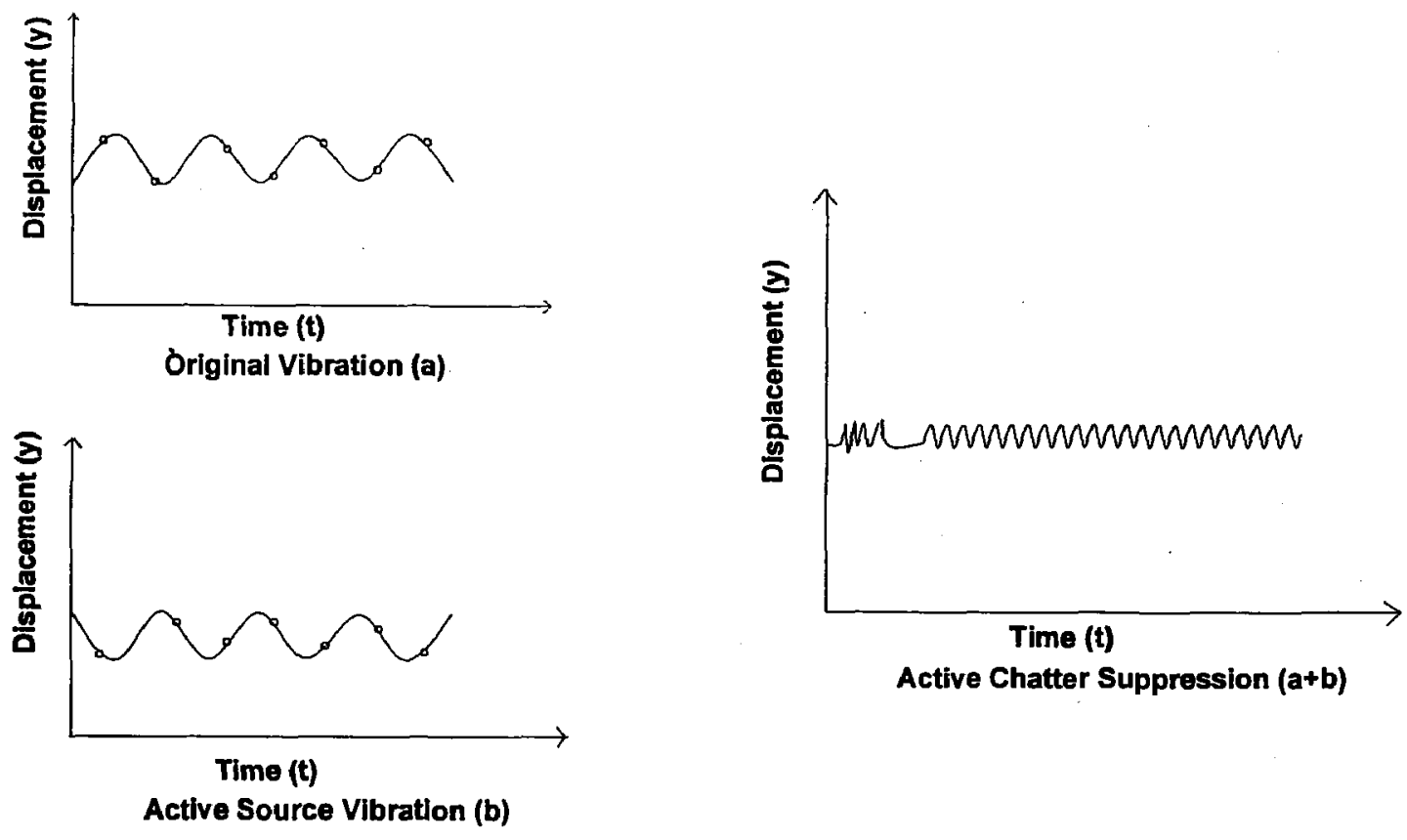

Figure 3.1: Active Chatter suppression system 
An external source generates a vibration signal to counteract the undesirable vibration, therefore, reducing its amplitude. The success of this method depends on accuracy of the vibration from the external source, in both magnitude and phase.

Effort in chatter suppression can be categorized into passive and active methods. Usually passive methods suppress chatter by using energy absorbing dampers to consume the energy input or by changing the cutting conditions to reduce the energy generated during machining process. However, passive methods are not very effective and practical since they need to be replaced or tuned for different cutting conditions, making them less useful than active control methods [16].

In this study an active system will be investigated which has the inherent capability of suppressing chatter under varying conditions.

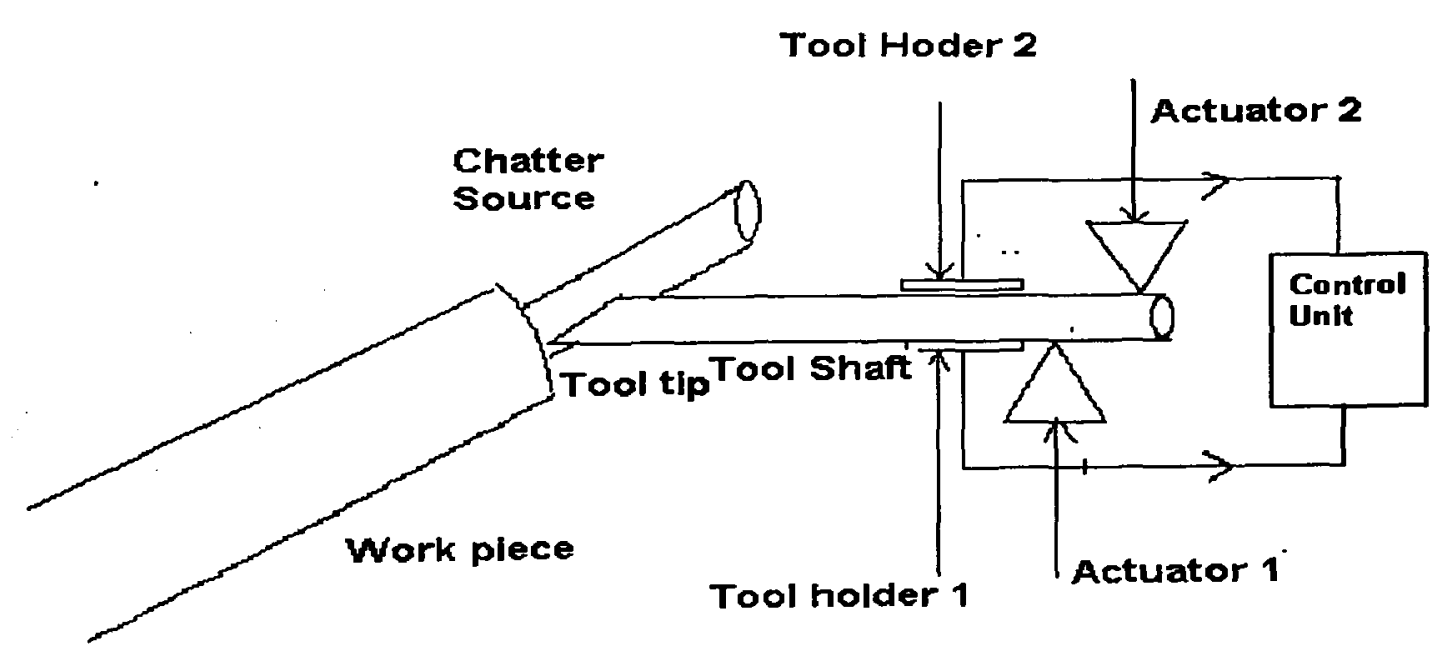

Figure 3.2: Simplified model of cutting process

Figure 3.2 shows a simplified model of a cutting process with an active chatter suppression. The speed of cutting is often limited by chatter and machine vibration induced by the cutting process. Chatter occurs when the machining process excites large- 
amplitude vibrations that are driven by regenerative variations in the cutting forces. The vibration signal of the cutting tool is detected by an accelerometer or a displacement pickup. The vibration signal is then passed to the actuator to produce a driving force for the cutting tool. The driving force provided by the actuator can be treated as an additional damping force for cutting tool. As a result, the extra damping force can effectively suppress the vibration of the cutting tool.

The goal of the proposed methodology here is to select, on-line, the optimum values for amplitude, phase angle, and frequency of the active vibration sources to achieve maximum chatter suppression. Figure 3.3 shows a simplified model of the cutting process as an input/output system.

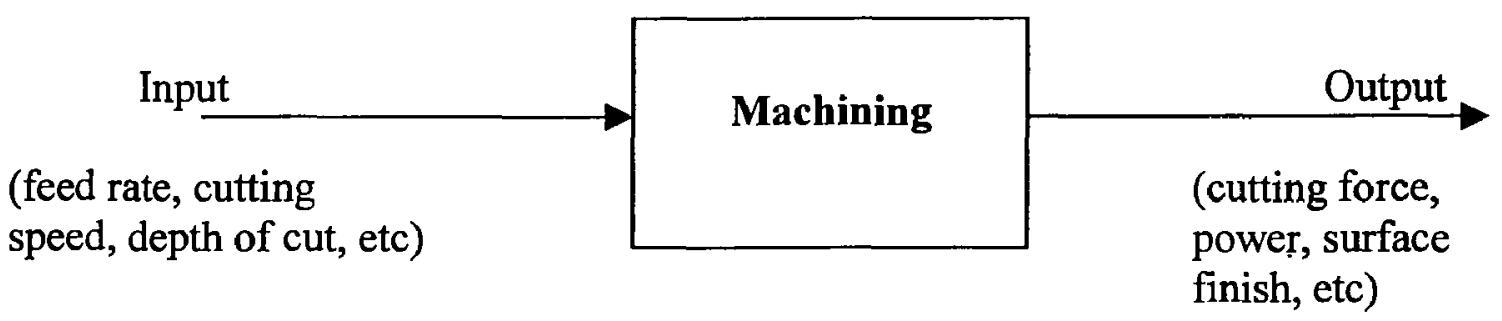

Figure 3.3: Machining Process as an input/output system

The inputs to the machining process are feed rate, cutting speed, depth of cut and output of the system during cutting process are cutting forces, power and the surface finish. Each of the output variables are constrained by upper bounds, which are dictated by criteria such as tool wear, work piece burn and machine capacity.

The block diagram of a chatter suppression system is shown in Figure 3.4. The external force $\mathrm{F}$ is generated through actuators for successful chatter suppression. 


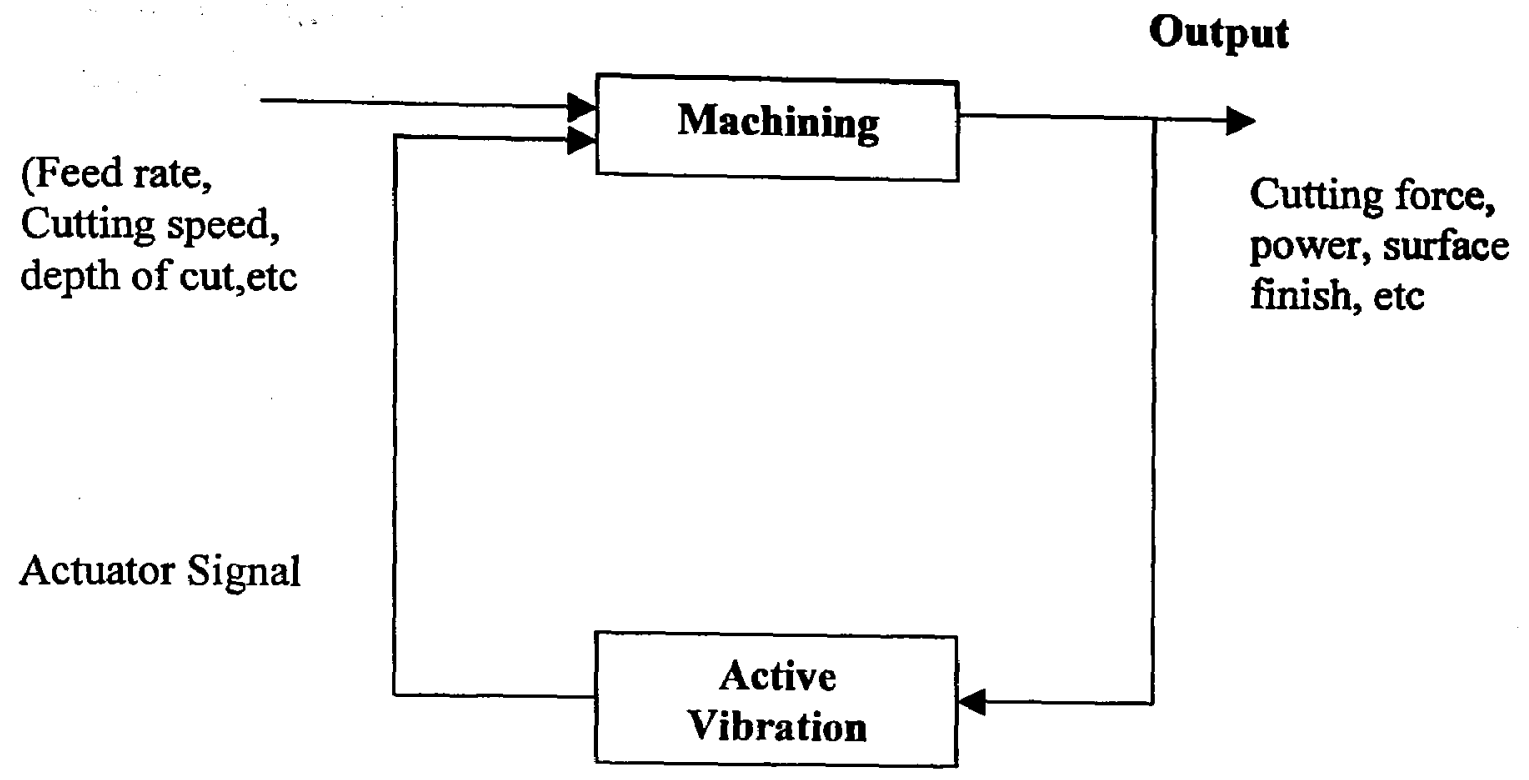

Figure 3.4: Chatter Suppression System

The parameter of the external forces provided by the actuator has to be determined online, and therefore adaptively changed in response to changes in cutting conditions, cutting tools, work piece material, and machine tool condition.

In the proposed methodology, the on-line selection of the optimum parameters for the active vibration system is based on the differentiable model of the cutting process. Considering the input/output model of the cutting process with the addition of the actuator forces, the following relation exist,

$$
\text { Output }=G(\text { Inputs, } t)
$$

Where $\mathrm{G}$ is the comprehensive dynamic model of the machining process relating inputs and outputs. If the amplitude of the vibration is of interest, as is the case in chatter, the output can be considered to be the amplitude of resulting vibration, i.e.,

$$
A_{i}=G(\text { Inputs, } t)
$$


Where, $\boldsymbol{A} \boldsymbol{i}$ is the amplitude of resultant vibration. In order to minimize these vibrations, actuator signal can be determined through an optimization method such as steepest decent, where

$$
X^{k+1}=x^{k}-\alpha^{k} \nabla G^{k}
$$

Where, $\nabla \mathrm{G}$ is the gradient of the dynamic model, $x^{k}, X^{k+1}$ are the values of the input variables in the $\mathrm{k}$ and $\mathrm{k}+1$ iteration and $\alpha$ is the size of the steps in the direction of minimization (Figure 3.5). The calculation of $\nabla G$ depends on availability of a differentiable model. Since a comprehensive physical model of machining process does not exist, this differentiable model is constructed using a neural network. The neural network provides

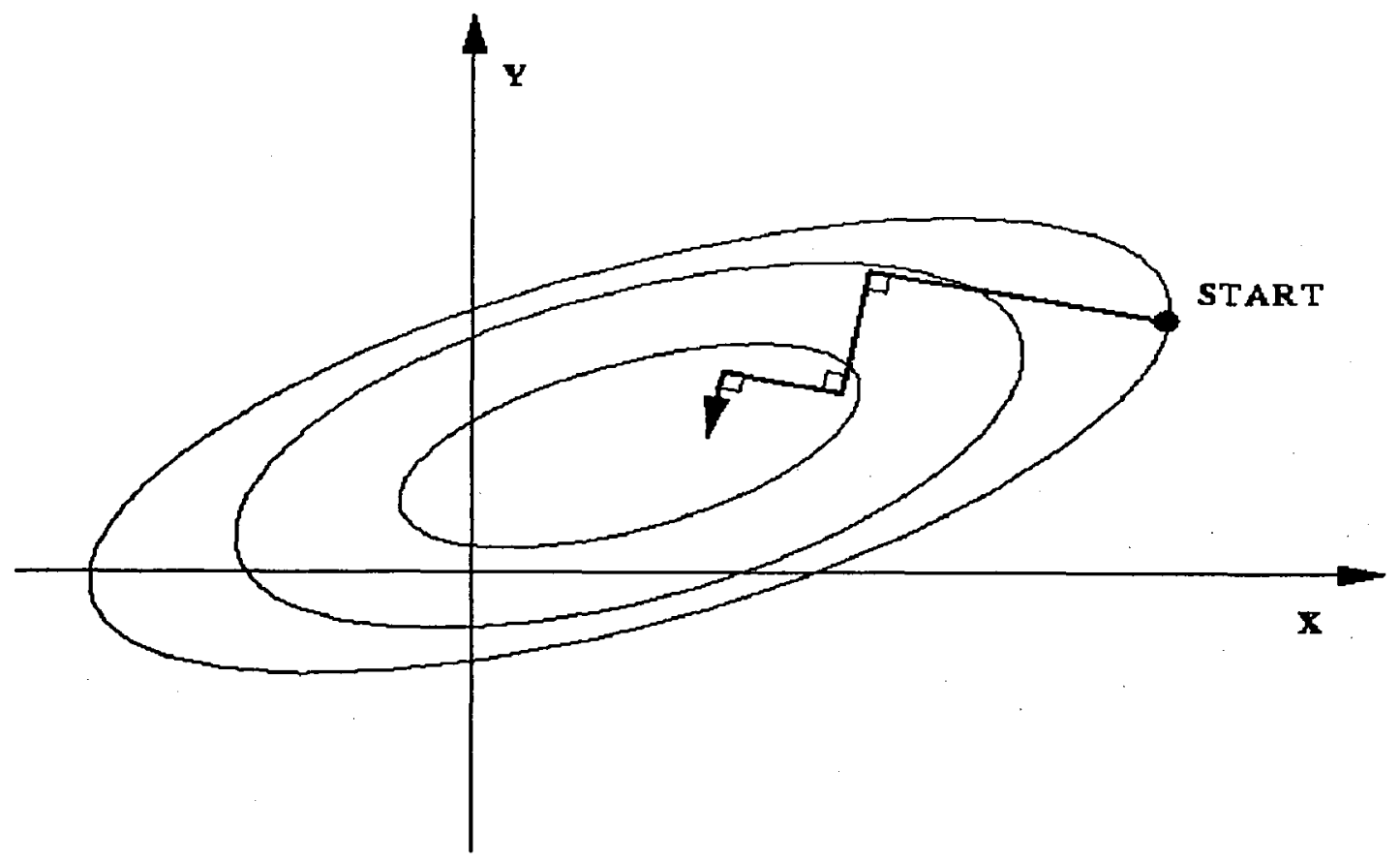

Figure 3.5: Steepest Descent Method 
a differentiable model of the cutting process and the dynamic effect of the tool-work piece interface.

The block diagram of chatter suppression system in Figure 3.6 shows the machining system, the differentiable model and actuator in the loop. The synthesis algorithm in the loop is used to adjust the actuator parameters on the basis of the operating conditions to actively suppress the response of the machine tool structure and stabilize the machining process.

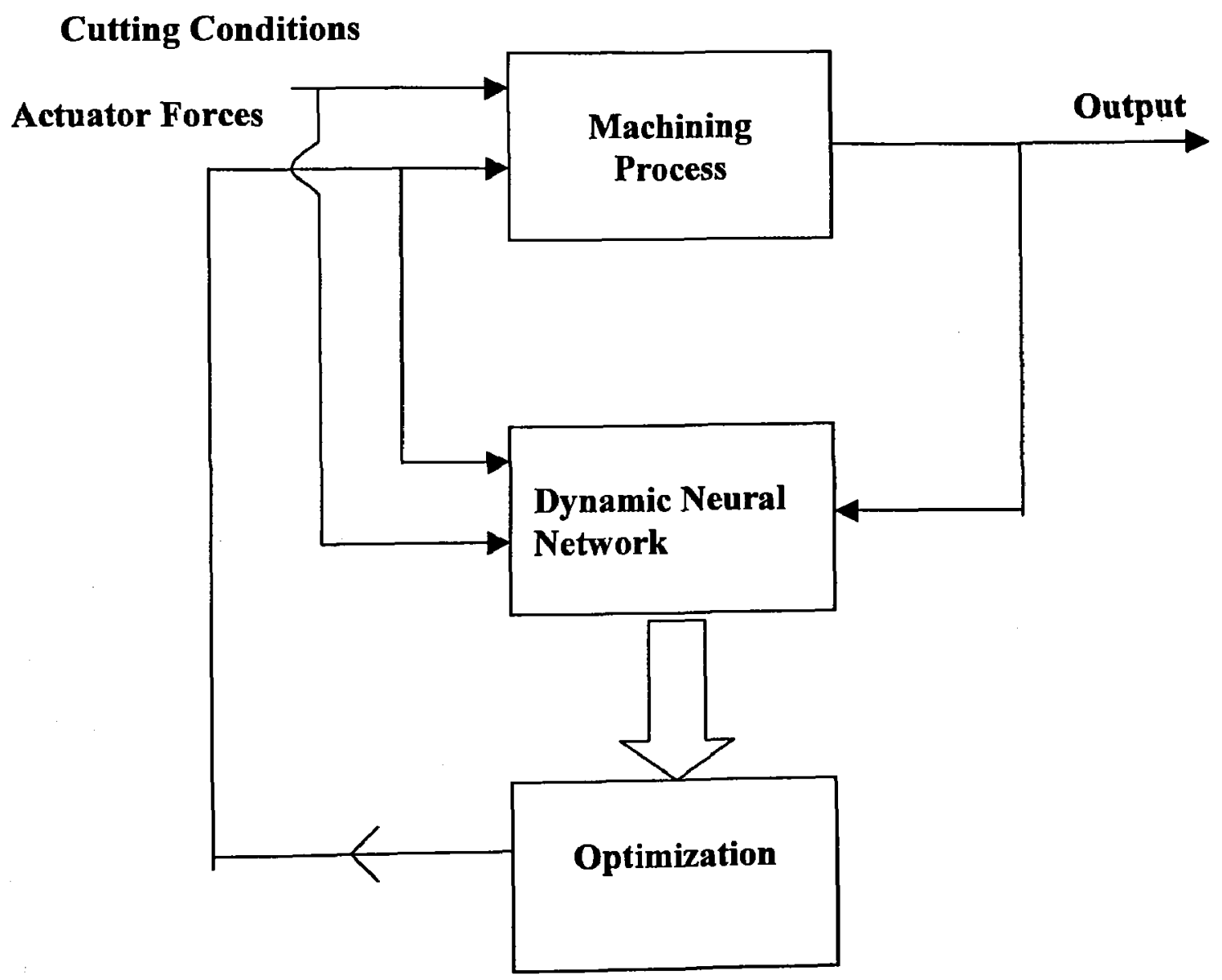

Figure 3.6: Active Chatter Suppression System 
The neural network used in this methodology is a multi-layered feed forward network. A quick overview of neural networks is presented here.

\subsection{Background on Neural Networks}

Neurobiologists have developed theories concerning how the cells in human brain operate and communicate. Artificial neural networks are mathematical models based on the observations of the human brain and were invented in 1943 by McCulloch and Pitts [17].

An ANN has a parallel, distributed, information-processing structure consisting of relatively simple processing elements similar to the neuro cells in the brain. Just like brain, supervised ANNs, learn by training repeatedly on a set of data. In supervised training, the neural network is shown both input data and the desired output data. After each trial, the network compares its own output with the correct output, corrects any deficiencies and tries again iterating until output errors reach an acceptable level [18].

\subsubsection{Multilayer Feed Forward Neural Networks}

A feed forward neural network is comprised of multiple layers of decision-making nodes called neurons. The first and last layers are the input and output layers respectively, and they are the only ones connected to the outside world. Usually there is a minimum of one hidden layer is located to every input in the first hidden layer and every output of the first layer is connected to every input in the subsequent layers ending at the output layer.

It is common to refer to feed forward neural network by the number of nodes present in each layer. For example, Figure 3.7 would be called a 3-2-3-2 network indicating 3 inputs and 2 outputs with 2 hidden layers of 2 and 3 nodes respectively. 


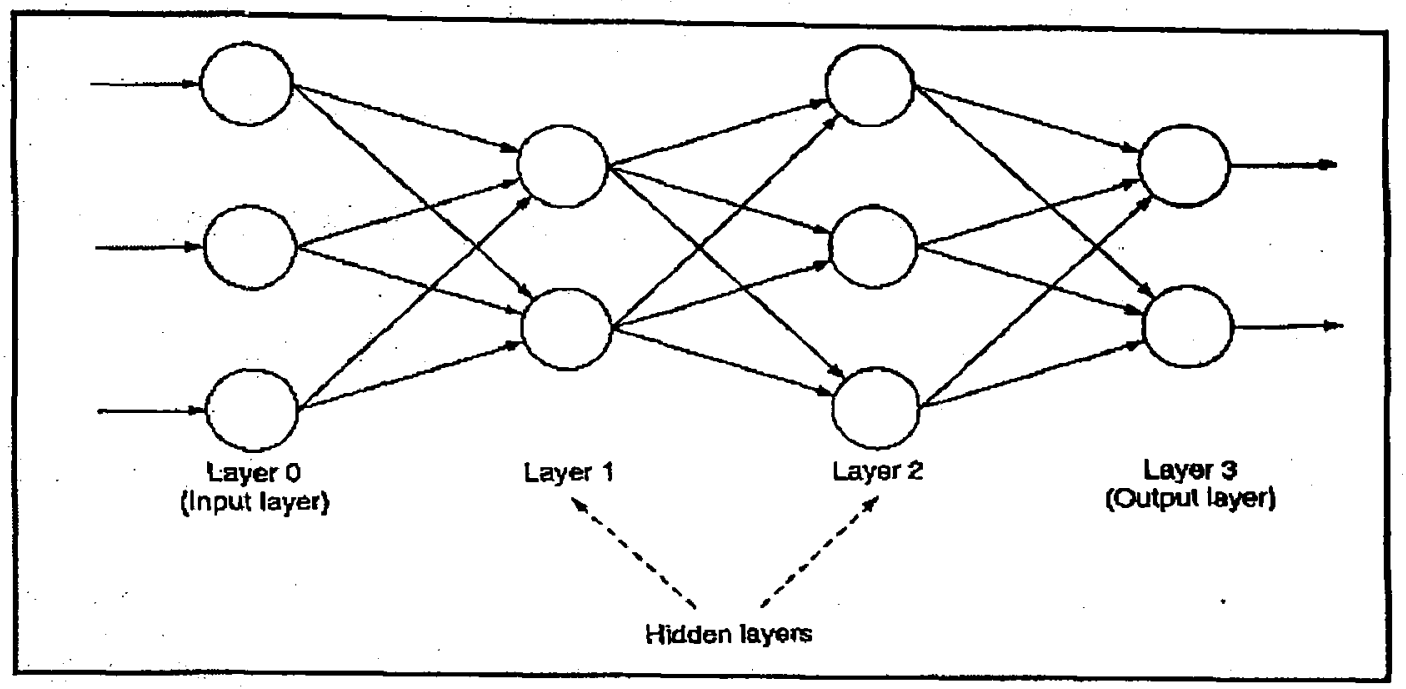

Figure 3.7 - Typical multi layer feed forward neural network architecture [17]

Each neuron can have multiple values of input but only one value can be output (figure 3.8). The outputs of the nodes are modified by weights before becoming inputs to the next layer.

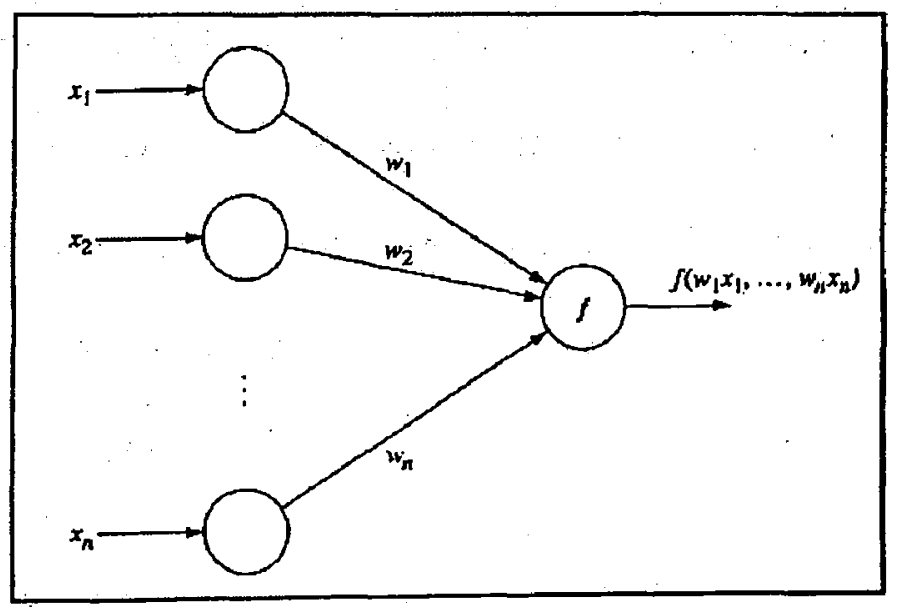

Figure 3.8- Neurons with multiple inputs and a single output [17]

The connections between layers modify the value of the output signals through a set of weights. Time for training increases with the number of layers and nodes in a network. If too many nodes are selected, the system may learn specific values and not be able to 
generalize a result. The recommended method for selecting the number of nodes is to start with a small number of nodes, one or two, and increase the number until the desired performance is reached or there are no improvements in error. It has been shown that a neural network with one hidden layer can approximate any function with a certain degree of error [19]. In practice, the amount of training required can be reduced in function with discontinuities by using a network with maximum of two hidden layers [20]. These networks are called universal approximators.

The input signal, $x_{i}$, are modified by the weights on the connections, $w_{i}$. The sums of the inputs are presented to a function at the nodes. A range of functions can be used at these decision-making nodes provided that they are non-linear and that their input range is all real numbers. The step function was used in early classification neural network models [17]. A ramp function has also been used and has the desirable property of being differentiable enables its use in the back propagation-training algorithm. The most popular function used in neural network is the sigmoid function.

The output of the sigmoid function is asymptotic and is typically limited to values between 0 and 1. Although the input range is not limited, it can also be normalized. Limits between 0.1 and 0.9 are used for normalizing data as it reduces the effect of the asymptotes. Normalization of all the data assures that all data have the same range, ensuring that no preference exists.

\subsubsection{Training}

Since there is no previous knowledge of the relationship of the system being analyzed, the neural network must be trained to interpret the data properly. Learning algorithms are used during training to modify the weights until the system gives the desired results within errors. There are also NNs which don't rely on examples to be trained (unsupervised NNs) but supervised NN were chosen for the model used in the proposed methodology. 


\subsubsection{Gradients of a Neural Network}

The learning procedures aim at driving the total error to zero or close to zero by suitable adjustment of the learning parameters. The main difficulty in learning algorithms for a Neural Network is that threshold function is non differentiable. Instead of using the threshold function, Rumelhart et al. [21] used a layered network in which the inputoutput behavior of the nodes is characterized by a sigmoid function and the transfer function is applied to all nodes. Such function can be trained by a learning algorithm called the generalized delta rule. This rule consists of an input pattern to the network and the calculating the output nodes with the current set of learning parameters.

The learning parameters of the NN are the weights and the node parameters. The network output pattern is then compared to the desired output patterns, and the error is calculated by computing the distance between the actual and desired output patterns. The procedure is repeated for all input-output pairs in the training set, and the total error is calculated.

The total error can be expressed as

$$
E=\frac{1}{2} \sum_{c} \sum_{i}\left(d_{i}-O_{i, n}\right)^{2}
$$

Where the index $\mathrm{i}$ represents summation over all outputs nodes in the network and $\mathrm{c}$ represents summation overall input-output cases used for training. The quantity $d_{i}$ is the desired output at the ith output node and $\mathrm{O}_{i, n}$ is the actual output at the ith output node. The total number of layers in the networks is $n$. The learning procedure aims to drive the total error to zero or close to zero by suitable adjustment of the learning parameters. This essentially constitutes a minimization problem that the generalized delta rule attempts to solve using gradients techniques. The calculation of the error gradient with respect to the learning parameters is performed by propagating the error backwards through the network and involves simple local computations at nodes in the same layer, permitting parallel operation of all nodes in that layer.

Once the gradient is calculated, the learning parameters are adjusted using steepest descent procedure that has the advantage of being simple and requires only local 
calculation during parameters adjustments. The change in the learning parameters is made as follows:

$$
\Delta w_{i}=-\eta \frac{\partial E}{\partial w_{i}}
$$

Where $w_{i}$ are the learning parameters, $E$ is the error and $\eta$ is the positive step size.

The gradients are computed only by local calculations so that updating the parameters can be done in parallel.

The Neural network is trained so that application of a set of inputs produces the desired set of outputs. Each such input (or output) set is referred to as a pattern. Training is accomplished by sequentially applying inputs, while adjusting network weights according to a predetermined procedure. During training, the network weights gradually converge to values such that each input vector produces the desired output vectors.

The most common training algorithms used is the backward propagation of the total error to calculate the error of the previous layers and nodes. Feed forward networks that used this training method to change the weights are sometimes mistakenly called back propagation networks, which indicate its popularity as training method. Once the error at each node has been calculated, a decision must be made in how to adjust the weight such that the overall error is minimized. The simplest back propagation algorithms change one weight at a time, and then recalculate the network in the opposite direction. This iterative process is time consuming. The conjugate gradient method improves on the basic algorithm by changing the weight in a direction such that the error is reduced.

\subsubsection{Validation}

In order to measure the success of the training, the generalization ability of the network is tested on previously unseen data. Generalization capabilities can be viewed as the ability to correctly predict outcomes from data within the range of the training set, but not included in the training set, called a test set. A method used to create a test set is to 
generate a training set and randomize the order of the training patterns and finally remove and save a percentage ( 10 to $25 \%$ depending on the total number of patterns) in a separate file. A network is said to have good generalization capabilities if similar error is achieved with the training and test set, then it is possible that the network has learned specific patterns and not the overall trends. This is likely to occur if too many hidden nodes are present or if the network has been over trained.

To ensure that overtraining does not occur, a training method, which continuously compares the training error to the test error, is commonly used. The training and test error are either continuously calculated after each successive training epoch or the test error can be calculated on predetermined error increments. The weights associated with each test error calculation are also stored. Using these methods, two distinct trends can be seen for the training and test error. (Figure 3.9)

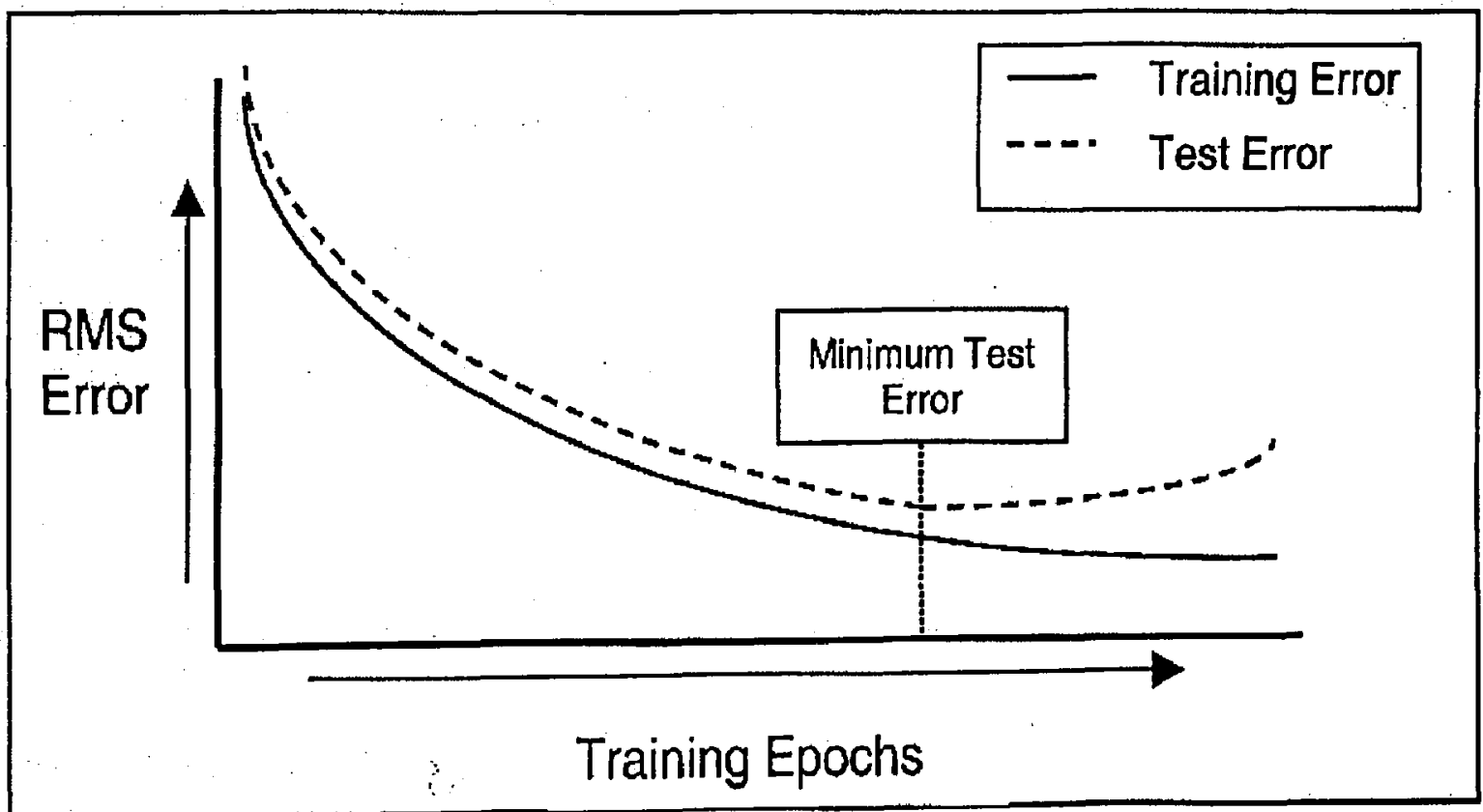

Figure 3.9: Training and test error as a function of training iterations [17] 
The training error can be seen to decrease as more training iterations are performed. The test error initially can be seen to decrease until a minimum value is reached. Training iteration beyond the test error minimum reduced the generalization ability of the network as can be seen by the increasing error for the test sets. The weights associated with the minimum error for the test sets should be used for comparison of different network architectures.

\subsection{Off-Line vs. On-line Training}

The brief introduction to neural networks presented can be applied to two distinct categories of neural networks: off-line and on-line.

An off-line network, the weights and connections are established during training prior to operation and do not vary. The quality and variety of data included in the training set is a very important factor in determining how well the network will perform. Unlike a off-line networks, an on-line network receives no training prior to its operation. Without prior training, the network is capable of adjusting its weights and connections to match a particular data set. These networks do not require training sets or time consuming training of a static network. The ability to change makes dynamic networks more suitable to learn changing data and be able to forecast future outcomes.

A neural network combining off-line and on-line neural networks could be called Adaptive. Careful interpretation of the previous term must be used as network which can change the number of nodes or eliminate connections during training have also been called adaptive [17].

\subsubsection{Dynamic vs. Static Neural Network}

A static neural network models input-output relationships which are not time dependent. A dynamic neural network, on the other hands models an input-output relationship which depends on time. 
There are different dynamic neural networks. One of the simplest ways to construct a dynamic neural network is by using tapped delay lines.A delay-line tap extracts a signal output from somewhere within the delay line, optionally scales it, and sums it into the output. A tap may be interpolating or non-interpolating. A non-interpolating tap extracts the signal at some fixed integer delay relative to the input. Thus, a tap implements a shorter delay line within a larger one, as shown in Figure 3.10.

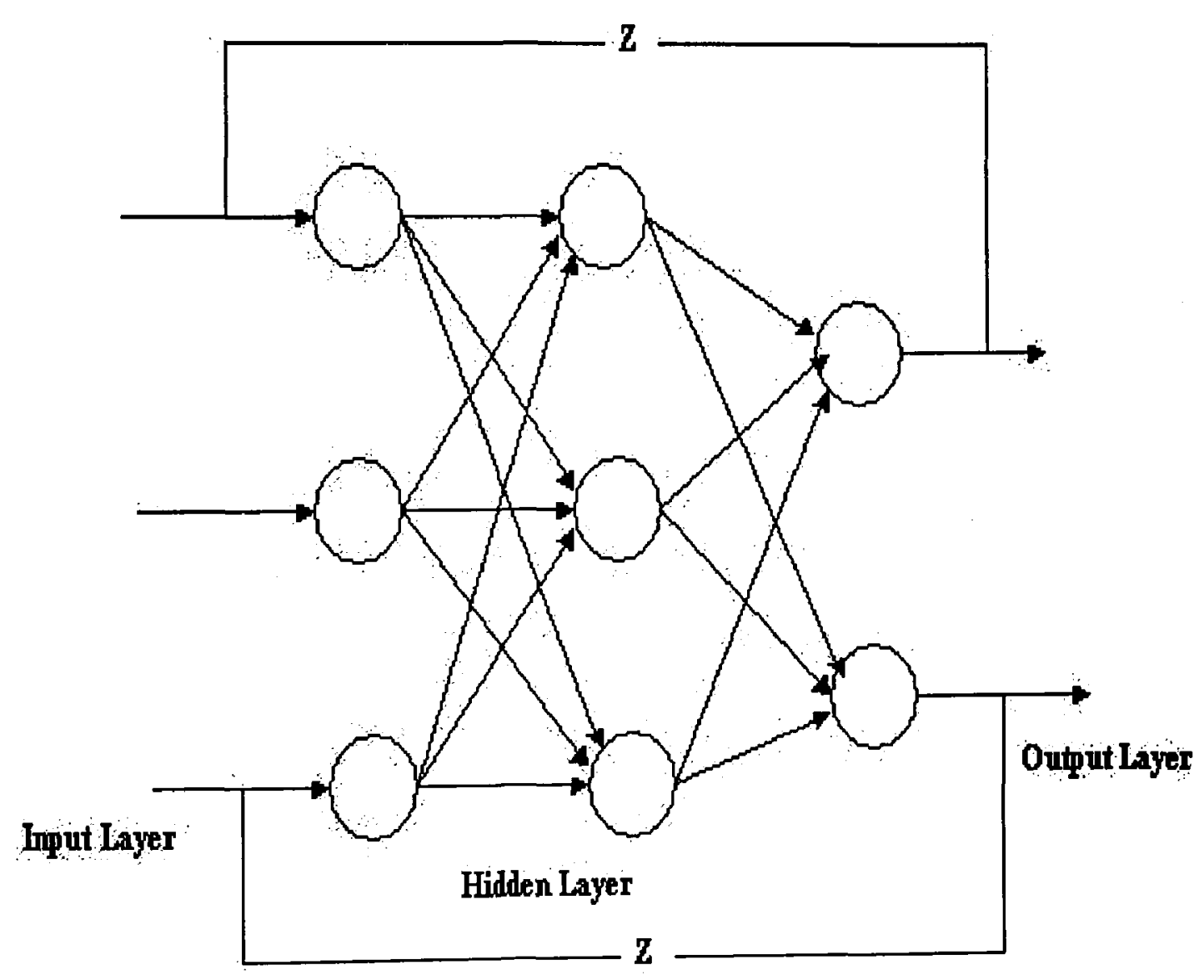

Figure 3.10: A Tapped delay Line

Since chatter is a dynamic process, a dynamic NN will be used for the model. Tapped delay lines will be used as the implementation method since it is possible to train the networks using training algorithms similar to back propagation. 


\subsection{Extracting Gradients Information from a Neural Network Model}

The purpose of using a neural network in the proposed chatter suppression is to provide gradient information necessary for minimizing chatter (Figure 3.6). This gradient information can be extracted once the neural network has been adequately trained.

A generalized sigmoid function to describe the input-output behavior of a processing unit [22]. The input - output relation is given as

$$
O_{i, k}=\frac{\theta_{i, k}}{1+e^{-\beta_{i, k} \text { net, }}}
$$

Where, $O_{i, k}$ is the output of the $\mathrm{i}_{t h}$ node in $\mathrm{k}_{t h}$ layer and $n e t_{i, k}$ is the input to the $\mathrm{i}_{t h}$ node in $\mathrm{k}_{t h}$ layer. $\theta_{i, k}$ controls the saturation value of the processor output and $\beta_{i, k}$ controls the steepness of the sigmoid function [23]. The input to a node is given by

$$
n e t_{i, k}=\sum_{j}\left[w_{i, j, k} O_{j, k-1}\right]+t_{i, k}
$$

Where, $\mathrm{w}_{i, j, k}$ weight between jth node in $(\mathrm{k}-1)_{t h}$ layer to $\mathrm{i}_{t h}$ node in $\mathrm{k}_{t h}$ layer and $\mathrm{t}_{t h}$ is the threshold of the $\mathrm{i}_{t h}$ node in the $\mathrm{k}_{t h}$ layer.

For a given input-output case, the error is defined as

$$
E=\frac{1}{2} \sum_{j=1}^{q}\left(d_{j}-O_{j, n}\right)^{2}
$$

Where $n$ is the total number of layers in the network and $q$ is the total number of output nodes. The error is calculated during a forward pass of the network, using the current values of the learning parameters [22]

Learning relies on minimizing $E$ by suitable adjustments of the learning parameters 
$\mathbf{w}_{i, j, k}, \mathbf{t}_{i, k}, \theta_{i, k}$ and $\beta_{l, k}$. This requires calculation of the derivative of $E$ with respect to the learning parameters. From (1) and (2) the following partial derivatives can be calculated;

$$
\begin{gathered}
\frac{\partial n e t_{i, k}}{\partial w_{i, j, k}}=O_{i, k-1} \\
\frac{\partial n e t_{i, k}}{\partial t_{i, k}}=1 \\
\frac{\partial O_{i, k}}{\partial \theta_{i, k}}=\frac{O_{i, k}}{\theta_{i, k}} \\
\frac{\partial O_{i, k}}{\partial \beta_{i, k}}=O_{i, k}\left(1-\frac{O_{i, k}}{\theta_{i, k}}\right) n e t_{i, k} \\
\frac{\partial O_{i, k}}{\partial n e t_{i, k}}=O_{i, k}\left(1-\frac{O_{i, k}}{\theta_{i, k}}\right) \beta_{i, k}
\end{gathered}
$$

All partial derivatives in (3.13) can be calculated during the forward pass. Once a feed forward NN is adequately trained, it can be used to calculate the gradients of output with respect to inputs. The network has $p$ inputs nodes, $q$ output nodes, and $n$ layers.

In order to calculate the gradients of the NN model, calculate all the $\frac{\partial y_{1}}{\partial z_{j}}$

Where $y_{i}$ are the outputs and $z_{j s}$ are the inputs to the neural network.

Define:

$$
\gamma_{i, s, k}=-\frac{\partial y_{i}}{\partial n e t_{s, k}}
$$

Where, net $t_{s, k}$ is the input to node $s$ in layer $k$, and $y_{i}$ is the output of node $i$.

Applying this to the output layer, using (8) 
$\gamma_{i, l, n}=-\frac{\partial y_{i}}{\partial n e t_{i, n}}=-y_{i}\left(1-\frac{y_{i}}{\theta_{i, n}}\right) \beta_{i, n}$

For the $(n-1)$ th layer,

$\frac{\partial y_{t}}{\partial n e t_{s, n-1}}=\left(\frac{\partial y_{i}}{\partial O_{s, n-1}}\right)\left(\frac{\partial O_{s, n-1}}{\partial n e t_{s, n-1}}\right)$

Since $y_{i}$ depends only on net $_{i, n}$, the first partial on the right side of (3.16) can be written as

$\frac{\partial y_{i}}{\partial O_{s, n-1}}=\left(\frac{\partial y_{i}}{\partial n e t_{i, n}}\right)\left(\frac{\partial n e t_{i, n}}{\partial O_{s, n-1}}\right)=-\gamma_{i, i, n} w_{i, s, n}$

Thus for the $(n-1)$ th layer, the $\gamma$ 's can be written as

$\gamma_{i, s, n-1}=-\frac{\partial y_{i}}{\partial n e t_{s, n-1}}=\gamma_{i, i, n} w_{i, s, n}\left(\frac{\partial O_{s, n-1}}{\partial n e t_{s, n-1}}\right)$

For lower layers, the $\gamma^{\prime}$ 's can be written as

$$
\gamma_{i, s, k}=-\frac{\partial y_{i}}{\partial n e t_{s, k}}=-\left(\frac{\partial y_{i}}{\partial O_{s, k}}\right)\left(\frac{\partial O_{s, k}}{\partial n e t_{s, k}}\right)
$$

Since

$$
\frac{\partial y_{i}}{\partial O_{s, k}}=\sum_{r}\left(\frac{\partial y_{i}}{\partial n e t_{r, k+1}}\right)\left(\frac{\partial n e t_{r, k+1}}{\partial O_{s, k}}\right)
$$

where the summation extends over all nodes in the $(k+1)$ th layer, (3.19) can be written as

$$
\gamma_{i, s, k}=\left[\sum_{r} \gamma_{i, r, k+1} w_{r, s, k+1}\right] \frac{\partial O_{s, k}}{\partial n e t_{s, k}}
$$


All partials on the right side of (3.18) and (3.20) are available from the forward pass of the network (refer to (3.18) ). Equations (3.19) and (3.20) are used to propagate the values of $\gamma$

from the output to input layer of the network [22]. At the input layer;

$-\frac{\partial y_{i}}{\partial z_{j}}=\gamma_{i, j, 1}=\left[\sum_{r} \gamma_{i, r, 2} w_{r, j, 2}\right]\left(\frac{\partial O_{j, 1}}{\partial z_{j}}\right)$

\subsection{Signal Processing}

Figure 3.11 shows the NN used in the proposed methodology. This is a dynamic NN using time delayed line (TDL).

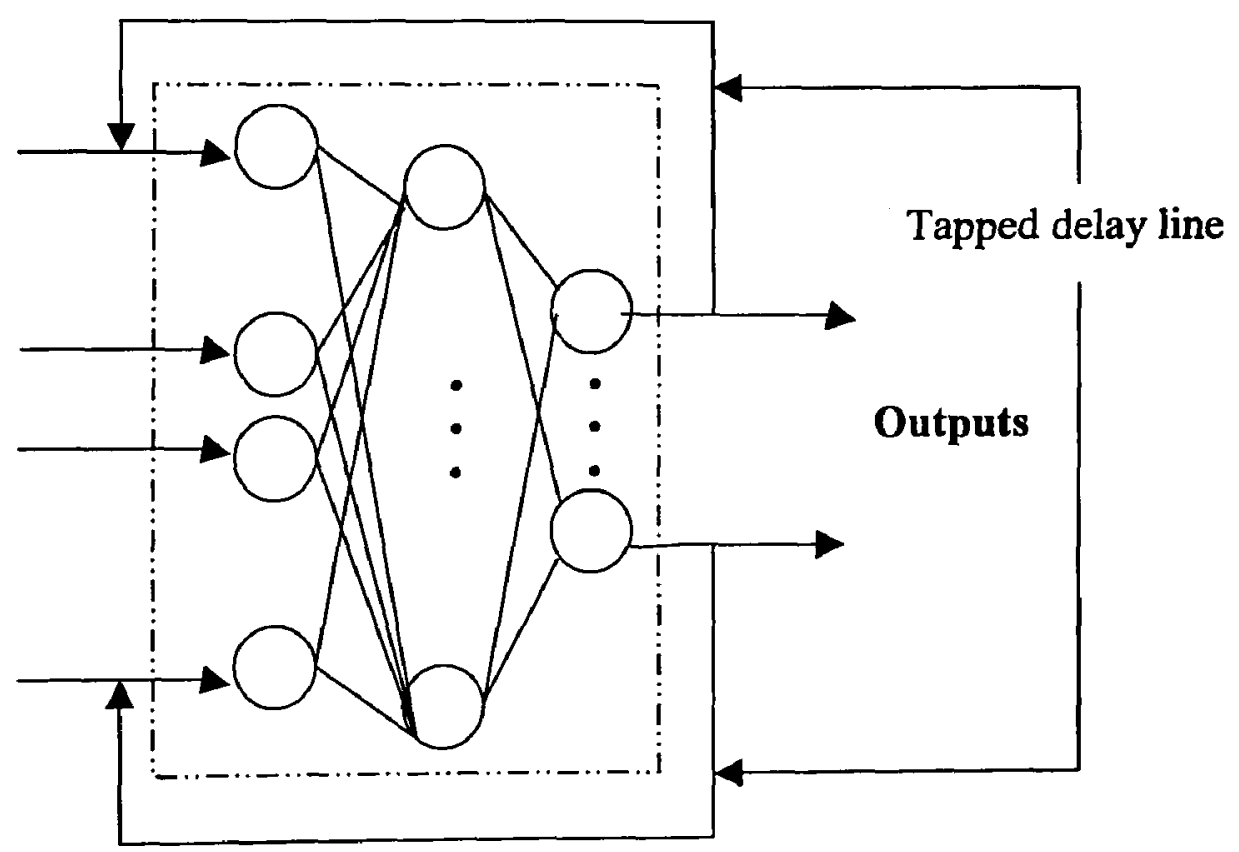

Figure 3.11: Dynamic neural networks with tapped delayed line

The success or failure of neural network training depends on the suitable presentation of the inputs and outputs. As for any dynamic system there are two choices available, time and frequency domain. 
Because of the nature of the chatter problem, modeling in frequency domain is more suitable. Several methods are available for presenting frequency domain information to a neural network. One method is to divide the frequency response to a predetermined number of bins and to presents the average amplitude in the bin as inputs Figure 3.12.

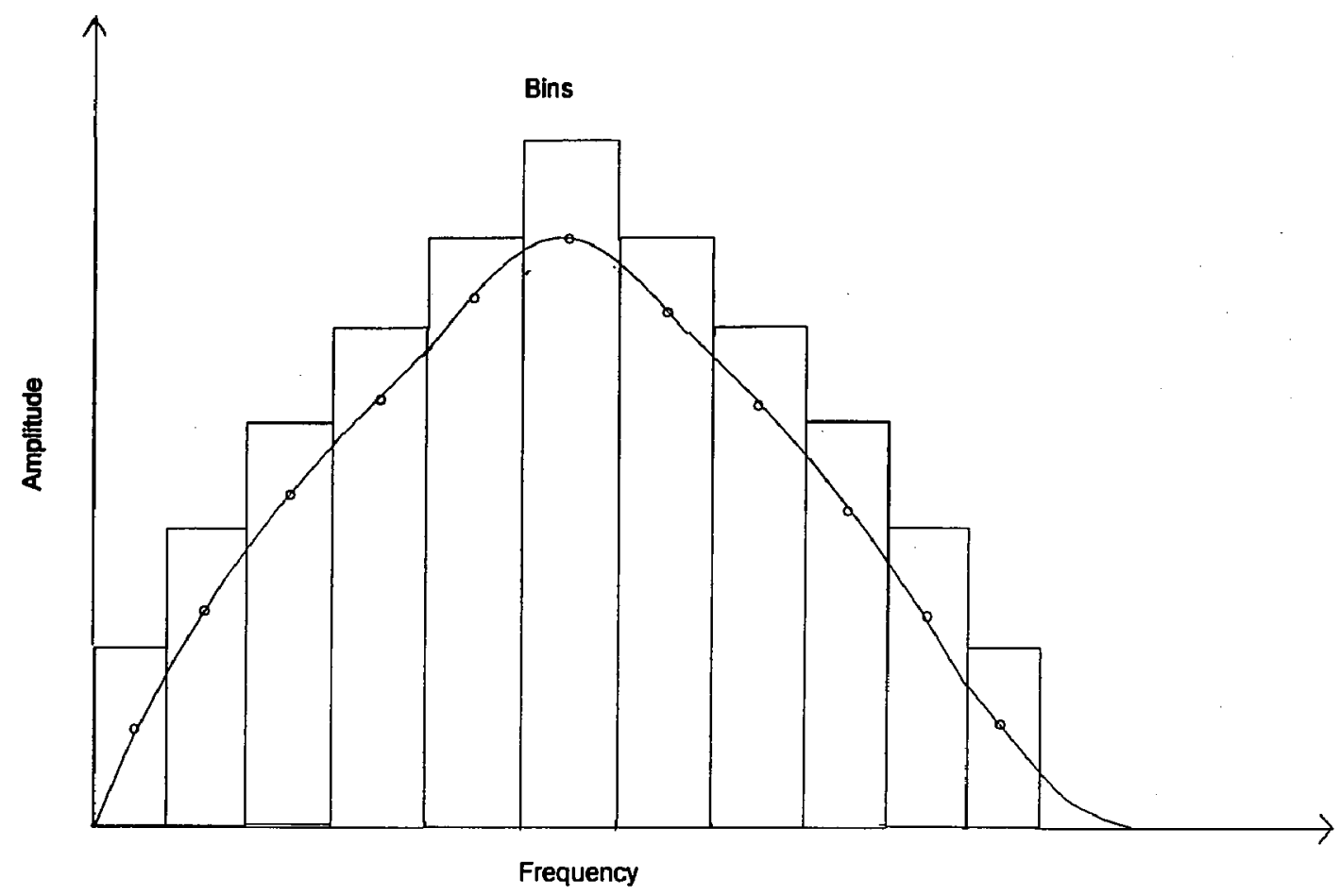

Figure 3.12: Representation of frequency response using finite number of bins

Another possibility is using the FFT coefficients as input. Since,

$$
X(k F)=\sum_{n=0}^{N-1} x(n T) e^{-j 2 \pi k F n t}
$$

Where, $\mathrm{N}$ is number of samples, $\mathrm{F}$ is spacing of frequency domain samples and $\mathrm{T}$ is sample period in the time domain. 
The obvious disadvantage of this method is the potentially high number of nodes required because of high number of coefficients. This will increase the processing time and can complicate the online implementation of the system. A third method, which facilitates the neural network training by reducing the number of parameters, is maximum entropy or all poles method. This method has the added bonus of reducing the effect of noise in the signals, and is briefly reviewed here.

\subsection{Maximum Entropy (all poles) Method}

Maximum entropy (all poles) method is also known as autoregressive (AR) spectral estimation. This method models the power spectrum by a series expansion in which the free parameters are all in the denominators of its terms; hence each term may represent a pole (corresponding to infinite power spectral density within an infinitely narrow frequency band). By contrast, Fourier analysis models the spectrum by a series expansion in which the free parameters are all in the numerators; hence each term in a Fourier series may represent a zero. All-poles models are particularly useful for analysis of spectra, which have discrete peaks [23].

MEM (also known as an all poles model) is an alternative to Fast Fourier Transform (FFT) analysis. The advantage of this signal processing method is that it can be quicker to run that FFT and it has the ability to fit sharp spectral peaks [23].

The number of coefficients selected determines the order or number of poles in the approximation. The number of poles used in an approximation determines the amount of features that can be identified. A smaller number of poles require less analysis time. If too many poles are selected phantom peaks may appear when compared to a FFT analysis. A computer program incorporating the code in numerical recipes was written to perform the analysis on all the data.

Using this method the power spectrum of the sensor measurement is estimated as 


$$
P(f)=\frac{a_{o}}{\left|1+\sum_{k=1}^{p} z^{-k}\right|^{2}}
$$

Here, $\mathrm{P}$ is estimate of power spectrum of the signal, $f$ is frequency, $\mathrm{a}_{\mathbf{k}}$ are coefficients of the autoregressive (AR) model of the measured signal, and $p$ is the order of the model determined by Akaike information criteria [24]. Power spectrum $P(f)$ is calculated using Levinson-Durbin's algorithm. Above equation shows that power spectrum of the sensor signal can be generated by $p$ coefficients where $\mathrm{p}$ is the order of AR model. This makes AR coefficients an efficient method of presenting frequency domain data to a neural network.

The number of coefficients selected determines the order or number of poles in the approximation. The number of poles used in an approximation determines the amount of features that can be identified. A smaller number of poles require less analysis time. If too many poles are selected phantom peaks may appear when compared to FFT analysis.

\subsection{Overview of the Proposed Method}

Figure 3.13 shows the flow diagram of the proposed chatter suppression system.

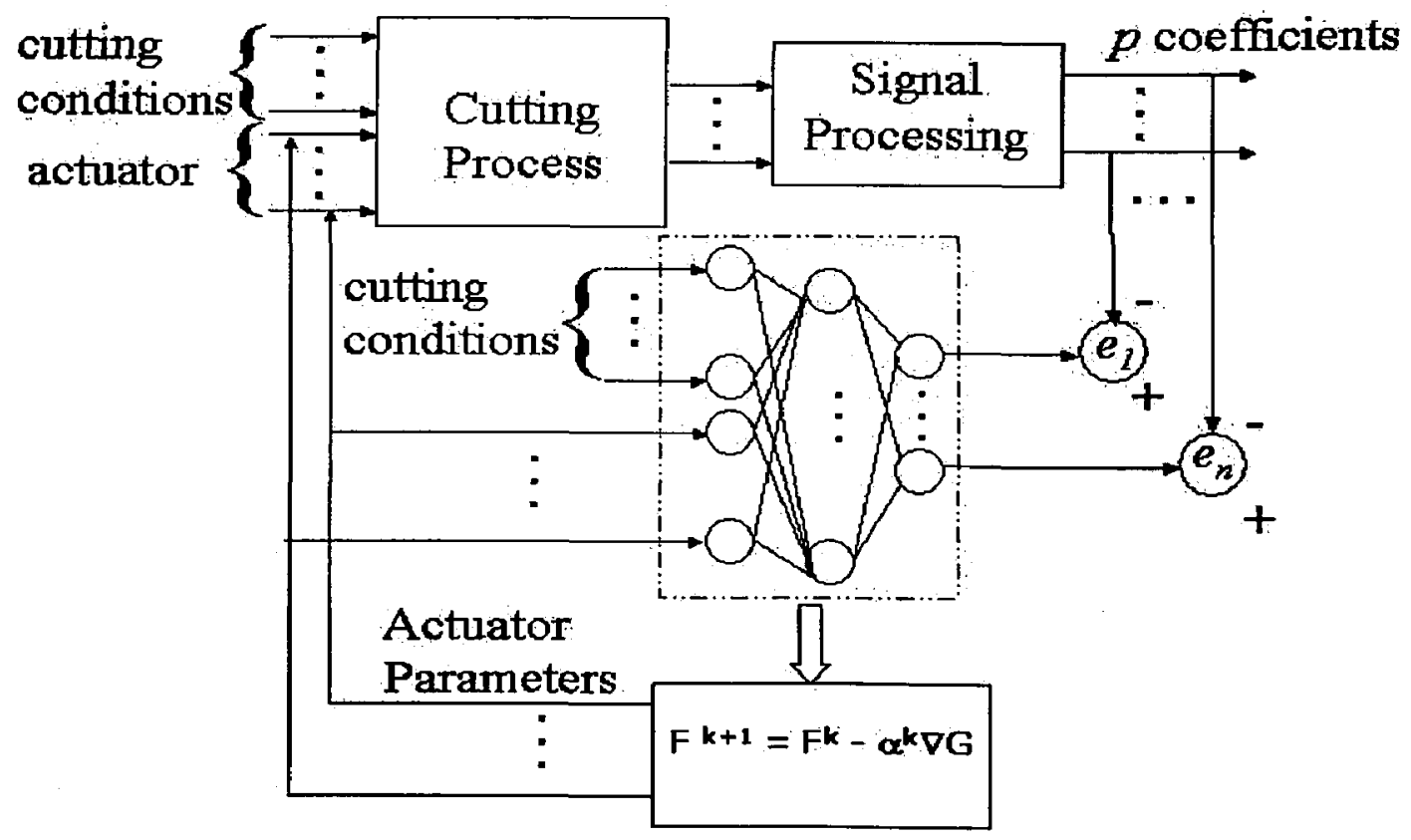

Figure 3.13: Adaptive feed forward chatter suppression system 
The active chatter suppression system is comprised of an active vibration source with continually adjustable parameters. The goal is to select, on-line, the optimum values for amplitude, phase angle, and frequency of the active vibration sources to achieve maximum chatter suppression

The neural network provides a differentiable dynamic model of the cutting process, including time delays between signals and the dynamic effect of the tool-work piece interface. A set of time delay lines ensures the capability of the neural model to capture the dynamics of entire system. This neural network model is used to calculate gradient values with respect to the parameters of the active vibration sources.

The goal is to generate an external force $F$ through an actuator to reduce chatter amplitude. In order to reduce chatter amplitude, actuator signal can be determined through an optimization method such as steepest decent, where

$$
X^{k+1}=x^{k}-\alpha^{k} \nabla G^{k}
$$

Where, $\nabla \mathrm{G}$ is the gradient of input/output functions, $x^{k}, X^{k+1}$ are the values of the variable in the $\mathrm{k}$ and $\mathrm{k}+1$ iteration and $\alpha$ is the size of the steps in the direction of travel The calculation of $\nabla G$ depends on availability of a differentiable model. From optimization method, the parameters of the active vibration source can be adaptively adjusted to reduce chatter amplitude and therefore extend the chatter stability.

Therefore, the proposed methodology acts as an automatic tuner for the actuators in order to suppress chatter. 


\section{Simulation Studies}

\section{Chapter 4}

\subsection{Introduction}

Chapter 3 introduced the proposed methodology to suppress chatter through a learning neural network model. A flow diagram of the proposed methodology was presented in Figure 3.10. An optimization module is used to synthesize a set of actuator parameters to optimally reduce the chatter. Simulation models to validate the proposed methodology is presented in this chapter.

\subsection{Simulation of Chatter}

In turning, the most significant cutting parameter, which is decisive for causing chatter, is the width of cut (b). For sufficiently small chip widths, cutting is stable, without chatter. By increasing $b$ chatter starts to occur at a certain width $b_{\text {lim }}$ and becomes more energetic for all values of $b>b_{\text {lim }}$ Figure 4.1. The value of $\mathrm{b}$ lim depends on the dynamic characteristics of the machine tool, work piece material, cutting speed and feed, and on tool geometry.

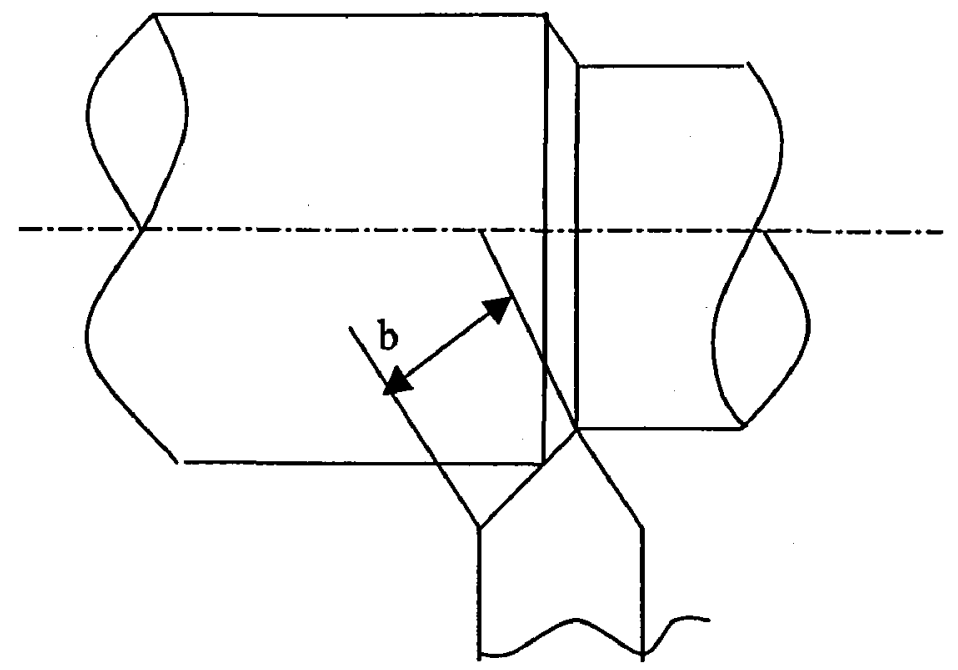

Figure 4.1: Simplified Model of Cutting Process 
The condition for the limit of stability is derived by Tlusty and polaek [5]. It is discussed with reference to the diagram in Figure 4.2.

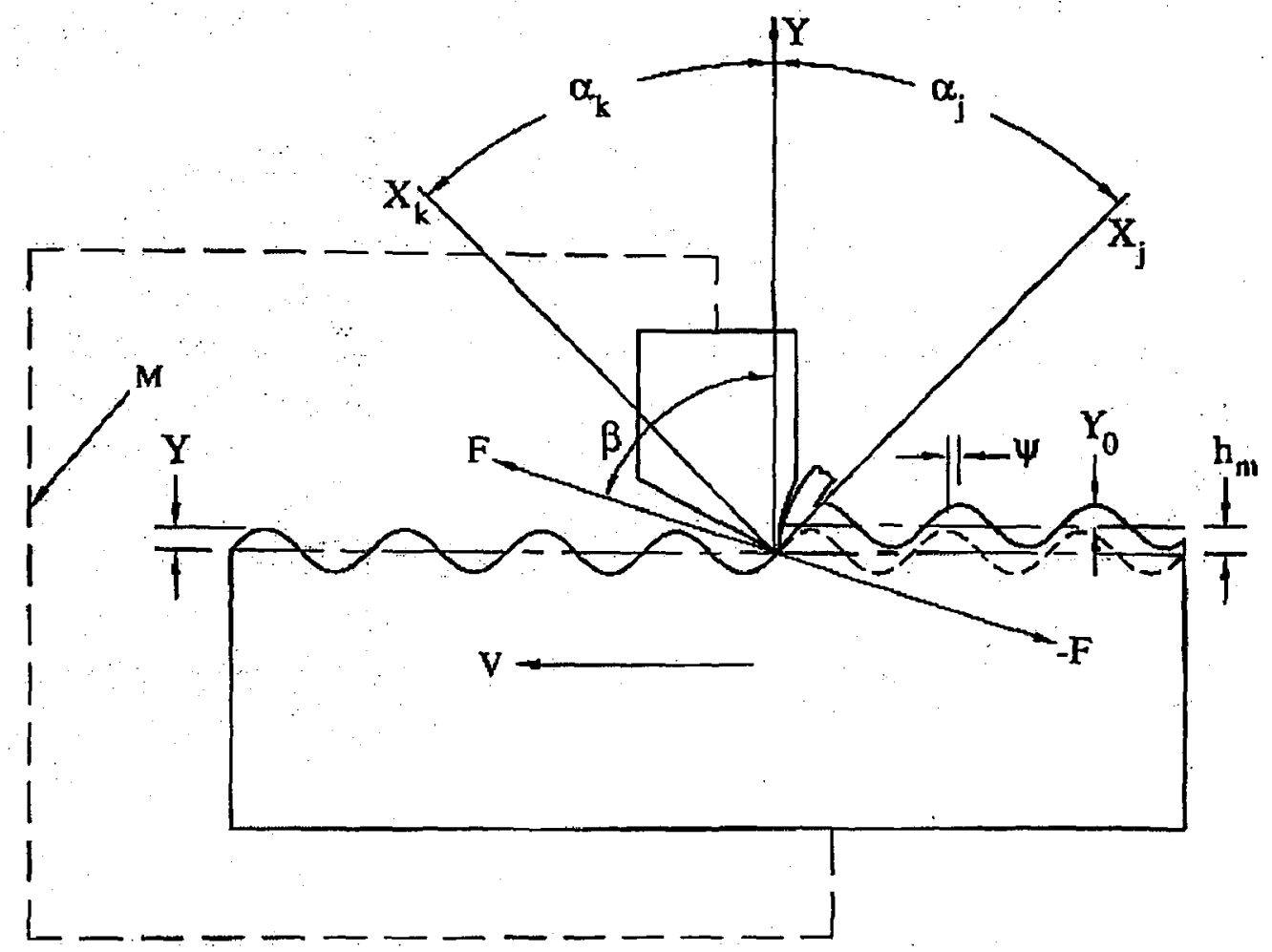

Figure 4.2: The Regeneration Diagrams Relating to Force, Surface Waviness and Vibration [5]

The structure of the machine tool is depicted as a frame with the work piece at one end and the tool at the opposite end. There is a relative cutting motion between the two, in the direction of the cutting speed $v$. The structure is a vibratory system that is characterized by the individual modes of vibration, each of which represents a freedom of the relative motion between the tool and work piece and has a particular shape (mode shapes). The vibration component, which is normal to the cut surface, produces undulations with an amplitude $Y_{0}$ in one cut, and in the subsequent cuts this component has amplitude $Y$. These amplitudes express both the magnitude and the mutual phase shift of the vibration, as well as undulations, in the two subsequent passes of cutting. The direction of the 
cutting force $F$ is inclined by the angle $\beta$ from $Y$, and the direction of the modes of vibrations are measured from Y by corresponding values of angles $\alpha$.

The process of self-excitation is a closed loop one in which the vibrations cause a force variation, and the variable force in turn produces vibrations. The force depends on vibrations in at least two previous passes [5] through variations of chip thickness,

$$
F=K_{s} b h
$$

Relationship between the amplitude F of the variable force and the amplitude of the chip thickness variation is [5]:

$$
F=K_{s} b\left(Y_{0}-Y\right)
$$

Where $b$ is the chip width, and $\left(Y_{0}-Y\right)$ is the chip thickness variation. The feedback relationship of vibrations caused by this force is in form of:

$$
Y=F G(\omega)
$$

Where $G(\omega)$ is the oriented transfer function (OTF) of the system.

From equation $4.2 \& 4.3$

$$
Y=K_{s} b G\left(Y_{0}-Y\right)
$$

After modification,

$$
\frac{Y_{o}}{Y}=\frac{1 /\left(K_{s} b\right)+G}{G}
$$

The condition of the limit of stability can be recognized as the condition for which vibrations do not decay or increase from pass to pass, i.e., the magnitudes $\left|Y_{o}\right|$ and $|Y|$ are equal: 


$$
\left|\frac{Y_{0}}{Y}\right|=1
$$

Combining equations 4.5 and 4.6 ,

$$
\left|\frac{1}{K_{s} b}+G\right|=|G|
$$

Equation 4.7 expresses the equality of the absolute values of two complex numbers. The function $\mathrm{G}(\omega)$ is complex, while $\left(\mathrm{K}_{s} b\right)$ is real. This condition has two parts:

$$
\begin{gathered}
\operatorname{Im}(\mathrm{G})=\operatorname{Im}(\mathrm{G}) \\
\frac{1}{K_{s} b}=\operatorname{Re}(\mathrm{G})= \pm \operatorname{Re}(\mathrm{G})
\end{gathered}
$$

Here the $(+)$ sign leads to $b=\infty$; the $(-)$ sign gives

$$
\frac{1}{K_{s} b}=-2 \operatorname{Re}(\mathrm{G})
$$

This is the actual condition for the limit of stability. The limit value of the chip width as follows [5]:

$$
\mathbf{b}_{\text {lim, }, r}=\frac{-1}{2 K_{s} \operatorname{Re}(G)_{\min }}
$$

The chip width $b$ is a positive number. Equation 4.10 can therefore only be satisfied for the negative part of the function $\operatorname{Re}[\mathrm{G}(\omega)]$. 
If chip widths $b<b_{\text {lim }}$ then cutting is stable: there is no self-excited vibration and if $b>b$ lim chatter will occur and grow. In practice, because of nonlinear ties in the phenomenon, the amplitude of chatter will stabilize at a finite value[5].

\subsection{Simulation Model for Turning}

The model used for the simulation of turning is shown in Fig 4.3(a)[5]. A system with two mutually perpendicular degrees of freedom $\mathrm{X} 1$ and $\mathrm{X} 2$ is used. A tool is attached to the mass of the system; it cuts an undulated surface and leaves another undulated surface behind. The normal to the cut surface is denoted Y. The simulation is carried out by proceeding in small time steps and following the motion of the system as it is determined by the differential equations of balance of forces in the direction $\mathrm{X} 1$ and $\mathrm{X} 2$ starting from some initial conditions.

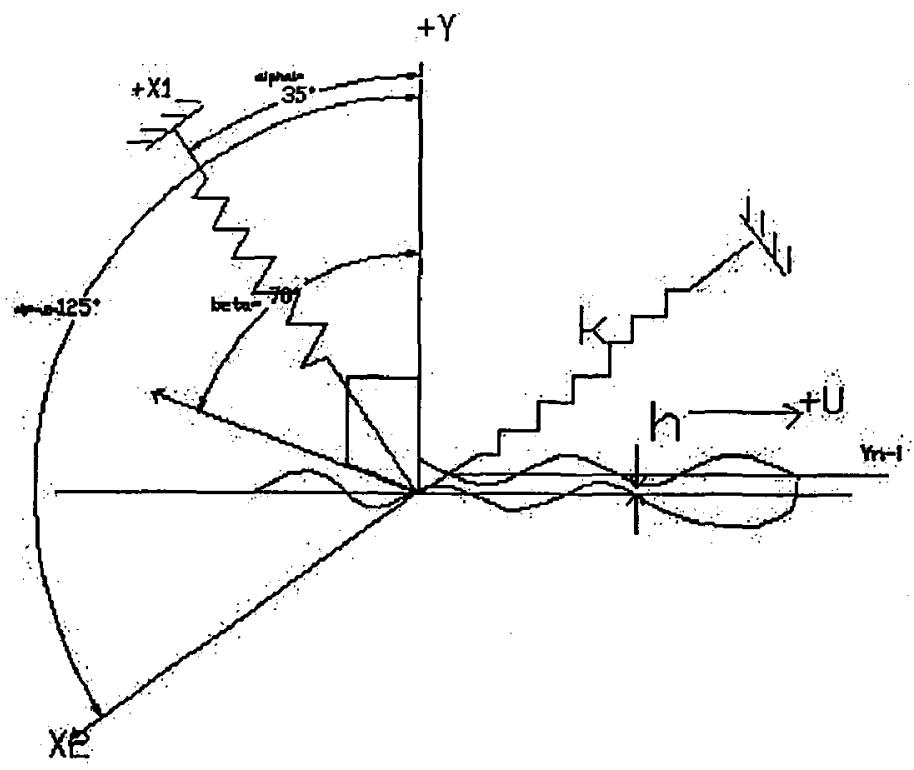

Figure 4.3(a): Model for Simulation of Turning [5] 
Subsequent passes of cutting follow one another after a period $T$, which correspond to one revolution of the work piece and contain $i$ time steps so that,

$$
t_{n}=n d t
$$

Where tn is the time from beginning of the simulation,

$$
\mathrm{T}=\mathrm{idt}
$$

Where, $T$ is the time period between subsequent passes. The average chip thickness is $h_{a v}$ and instantaneous chip thickness is usually taken as a difference of the tool positions in two subsequent passes;

$$
\mathrm{h}=\mathrm{h}_{\mathrm{av}}+\mathrm{y}_{\mathrm{n}-\mathrm{I}}-\mathrm{y}_{\mathrm{n}}
$$

As vibration grows more than one preceding cut may be involved. Figure 4.3(b) shows the model for simulation of turning. The cuts preceding the present cut reached lowest in the work piece material and denote such position $y_{\min }$.

$y_{\min }$ is the lowest of $y_{n-l}+$ hav, $y_{n-2 i}+2 h a v$, and so on.

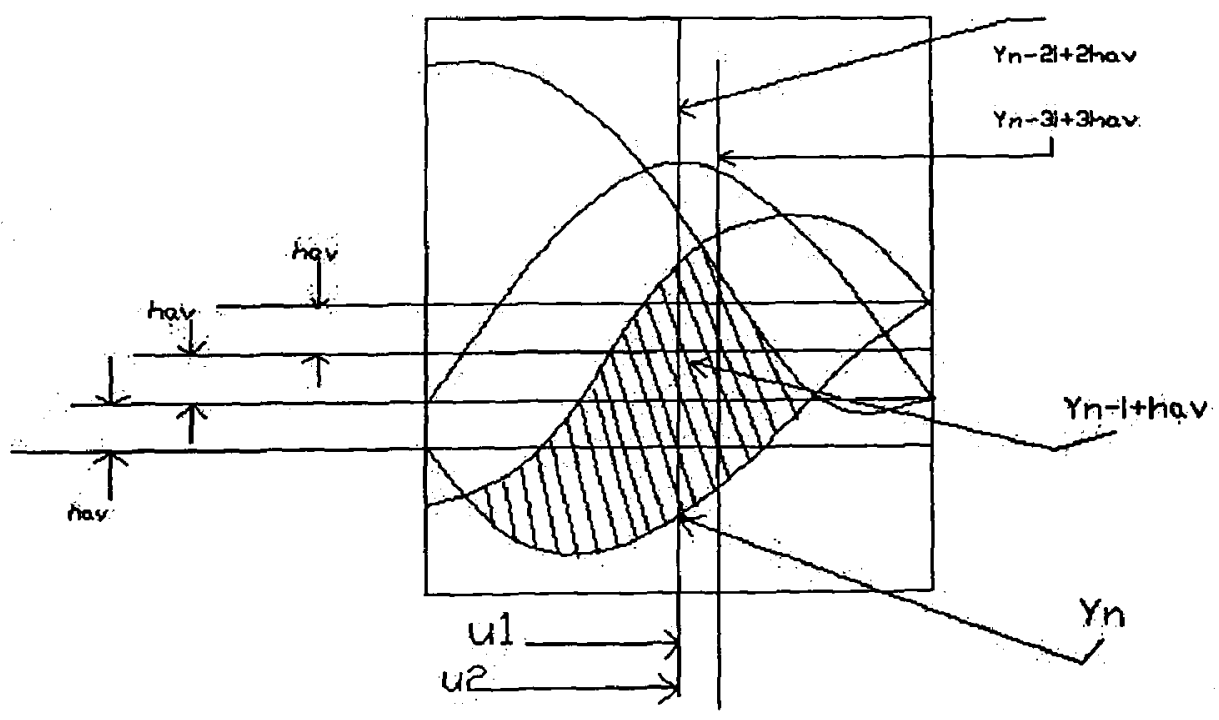

Figure 4.3(b): Model for Simulation of Turning [5]

The instantaneous chip thickness is, therefore,

$$
\mathrm{h}=\mathrm{y}_{\min }-\mathrm{y}_{\mathbf{n}}
$$

The material removed in the pass $y_{n}$ is indicated by the shaded area, and chip thickness at 
the distance $u_{1}$ is $h=y_{n-I}+$ hav $+y_{n}$, but at a distance $u_{2}$ it is $h=y_{n-3 i}+3$ hav $-y_{n}$. The cutting force at the instant $t_{n}$ is proportional to chip thickness $h$ and to chip width $b$ [5].

$$
\mathrm{F}_{\mathfrak{n}}=K_{s} b h
$$

but if

$$
F_{n}<0, \quad F_{n}=0
$$

Equation 4.16 expresses the basic non linearity. The force is proportional to chip thickness $h$, but if the tool moves above the surface, this is formally recognized by obtaining $h<0, F_{n}<0$, since the cutting force is never negative, this should not be accepted, instead force should be set to zero.

The force excites vibrations $\mathrm{X} 1$ and $\mathrm{X} 2$ by its components

$$
\begin{aligned}
& F 1=F_{n} \cos (\beta-\alpha 1) \\
& F 2=F_{n} \cos (\beta-\alpha 2)
\end{aligned}
$$

The equations of motion are;

$$
\mathrm{F}_{\mathrm{j}}=\mathrm{m}_{\mathrm{j}} \ddot{x}+\mathrm{c}_{\mathrm{j}} \dot{x}_{\mathrm{j}}+\mathrm{k}_{\mathrm{j}} \mathrm{x}_{\mathrm{j}} \quad \mathrm{j}=1,2,3 \ldots
$$

In the simulations, in each step $\mathrm{n}$, the acceleration $\ddot{x}_{j n}$ is calculated. Velocity is determined from equation 4.19 by integration and displacement is obtained by double integration. For $\mathbf{x}_{1}$

$$
\begin{gathered}
\ddot{x}_{1, \mathrm{n}}=\mathrm{F}_{1, \mathrm{n}}-\mathrm{c}_{1} \mathrm{x}_{1, \mathrm{n}}-\mathrm{k}_{1} \mathrm{x}_{1, \mathrm{n}} / \mathrm{m} \\
\dot{x}_{1, \mathrm{n}+1}=\dot{x}_{1, \mathrm{n}}+\ddot{x}_{1, \mathrm{n}} \mathrm{dt} \\
\mathrm{x}_{1, \mathrm{n}+1}=\mathrm{x}_{1, \mathrm{n}}+\dot{x}_{1, \mathrm{n}+1} \mathrm{dt}
\end{gathered}
$$


$\mathrm{x}_{2, n+1}$ can obtain in a similar fashion. It is then possible to express the tool motion in the $\mathrm{Y}$ direction as [48]

$$
\mathrm{Yn}=\mathrm{x}_{1, n} \cos \alpha_{1}+\mathrm{x}_{2, \mathrm{n}} \cos \alpha_{2}
$$

The system is linear as long as the tool does not leave the cut. Therefore for incipient vibration, equation 4.10 for the basic limit of stability width of chip $b_{\text {lim, } r}$ applies

$$
\mathrm{b}_{\mathrm{lim}, \mathrm{cr}}=\frac{-1}{2 K_{s} \operatorname{Re}(G)_{\min }}
$$

Where $\operatorname{Re}(\mathrm{G}) \min$ is the real part of the oriented transfer function between vibration $y$ and force $\mathrm{F}$, and most favorable condition between vibration in subsequent cuts. The parameters used for simulation is shown in Table 4.1

\begin{tabular}{|c|l|}
\hline $\mathrm{k}_{1}$ & $4.0^{*} 10^{7} \mathrm{~N} / \mathrm{m}$ \\
\hline $\mathrm{k}_{2}$ & $5.6^{*} 10^{7} \mathrm{~N} / \mathrm{m}$ \\
\hline $\mathrm{m}$ & $100 \mathrm{~kg}$ \\
\hline$\alpha_{1}$ & $30^{0}$ \\
\hline$\beta_{1}$ & $70^{\circ}$ \\
\hline$K_{s}$ & $2000 \mathrm{~N} / \mathrm{mm}^{2}$ \\
\hline $\mathrm{c}_{1}$ & $5320 \mathrm{~N} \mathrm{sec} / \mathrm{m}$ \\
\hline$c_{2}$ & $7480 \mathrm{~N} \mathrm{sec} / \mathrm{m}$ \\
\hline$\alpha_{2}$ & $120^{0}$ \\
\hline $\mathrm{h}_{\mathrm{av}}$ & $0.10 \mathrm{~mm}$ \\
\hline
\end{tabular}

\section{Table 4.1: Model Parameters for Turning}


Figure 4.4 shows the simulation result for $b=1.63 \mathrm{~mm}$. The vibrations are decreasing revolution after revolution. The magnitude of vibrations after four revolution is becoming more and more uniform and the case is stable

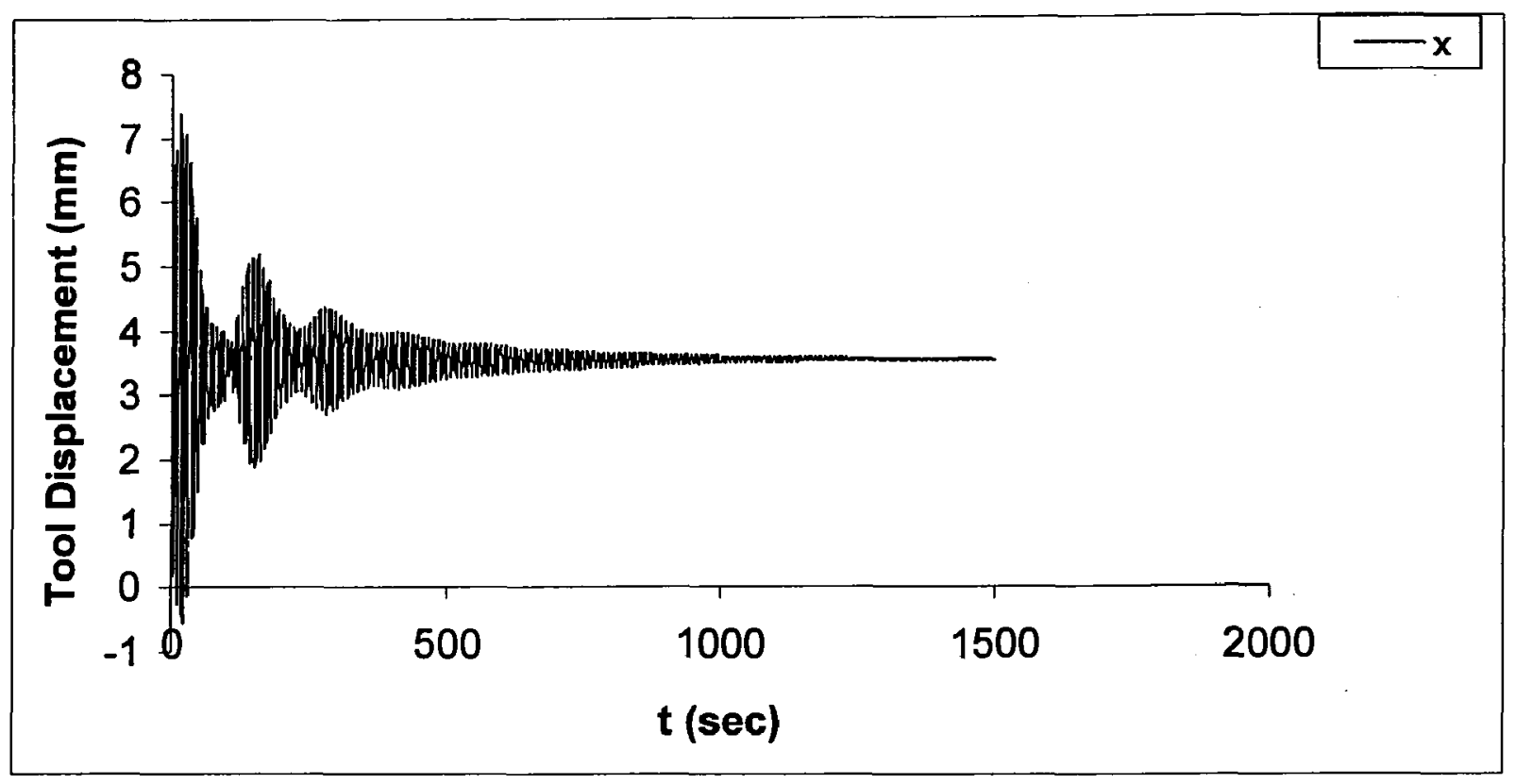

Figure 4.4: Turning Chatter Simulation for $b=1.63 \mathrm{~mm}$

Figure 4.5 shows the simulation result for $b=2.04 \mathrm{~mm}$ corresponding to $b$, the vibration

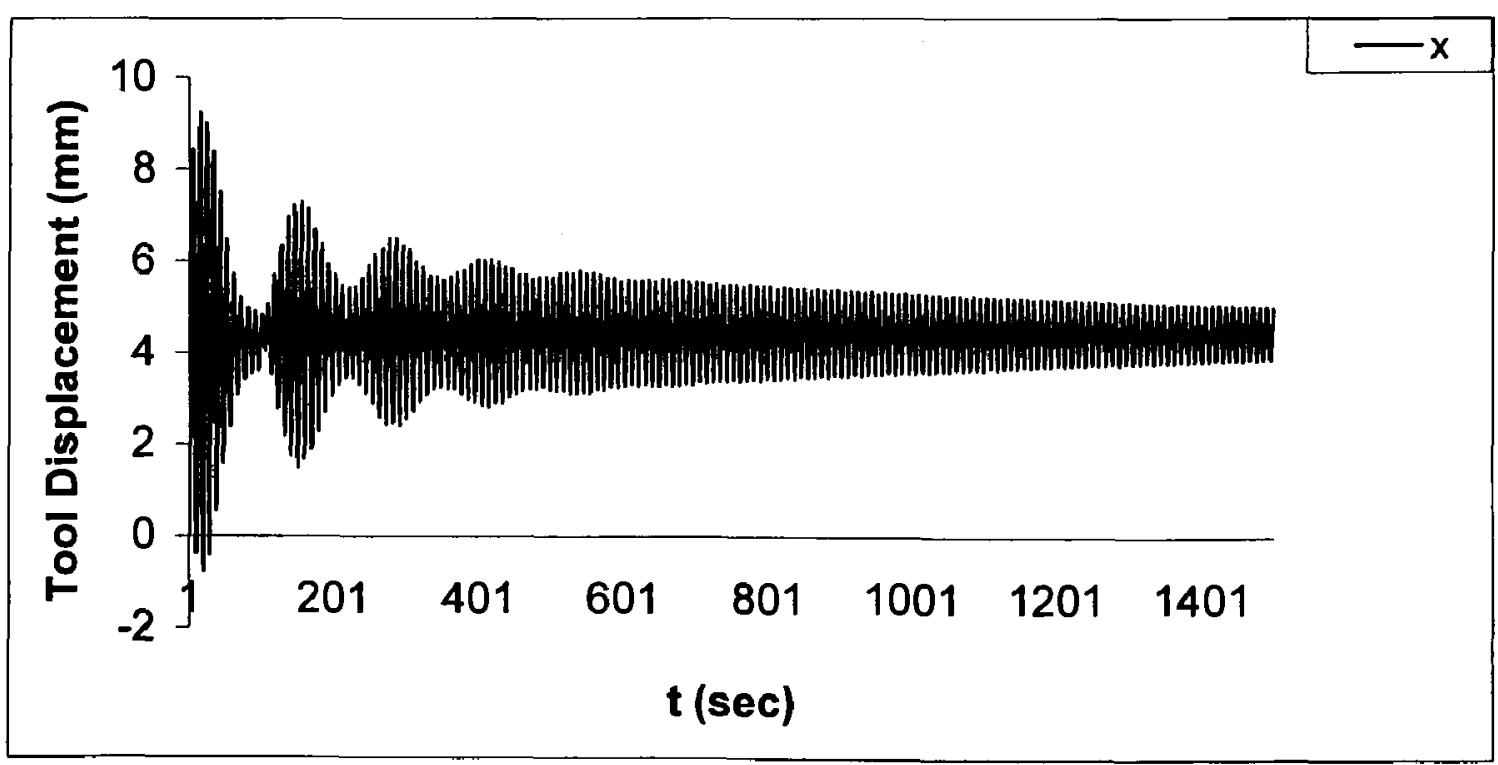

Figure 4.5: Turning Chatter simulation for $b=2.04 \mathrm{~mm}$ 
are becoming more and more uniform and, after a great number of revolutions they would develop a constant amplitude. The case is at the limit of stability. The magnitude of vibrations during the first five revolution is, on average, about $6 \mathrm{~mm}$ peak to peak (PTP).

Figure 4.6 shows the simulation result for $b=2.45$, corresponding to $b>b$ lim the vibrations are growing already during the first revolution, and in the fifth revolution their average magnitude is about $14 \mathrm{~mm}$, peal to peak (PTP).

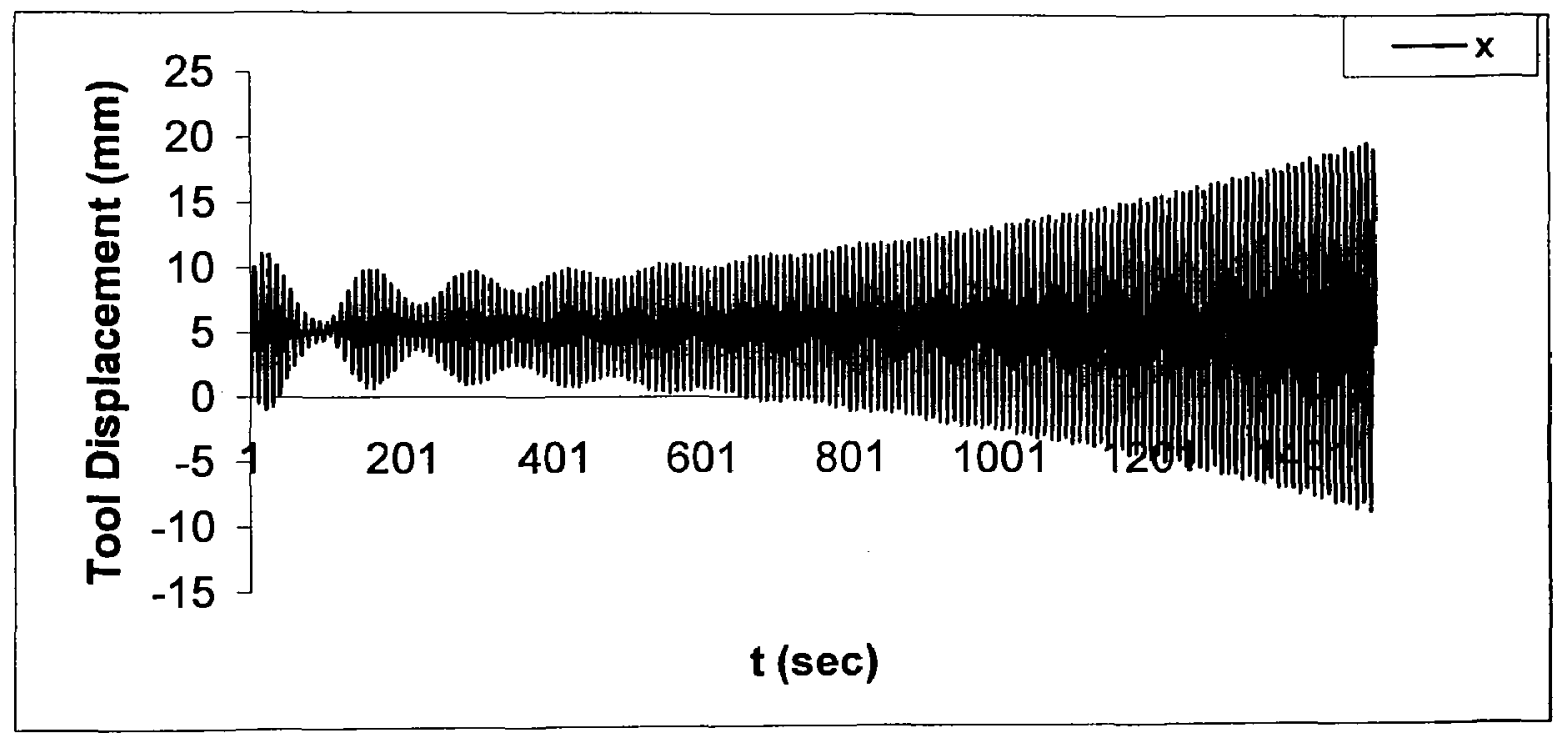

Figure 4.6 : Turning Chatter Simulation for $b=2.45 \mathrm{~mm}$

Figure 4.7 shows the simulation result for $b=3.06 \mathrm{~mm}$, the growth of vibrations is very fast. In the seventh revolution they reach an average value of about $80 \mathrm{~mm}$, peak to peak (PTP), and they grow still further. 


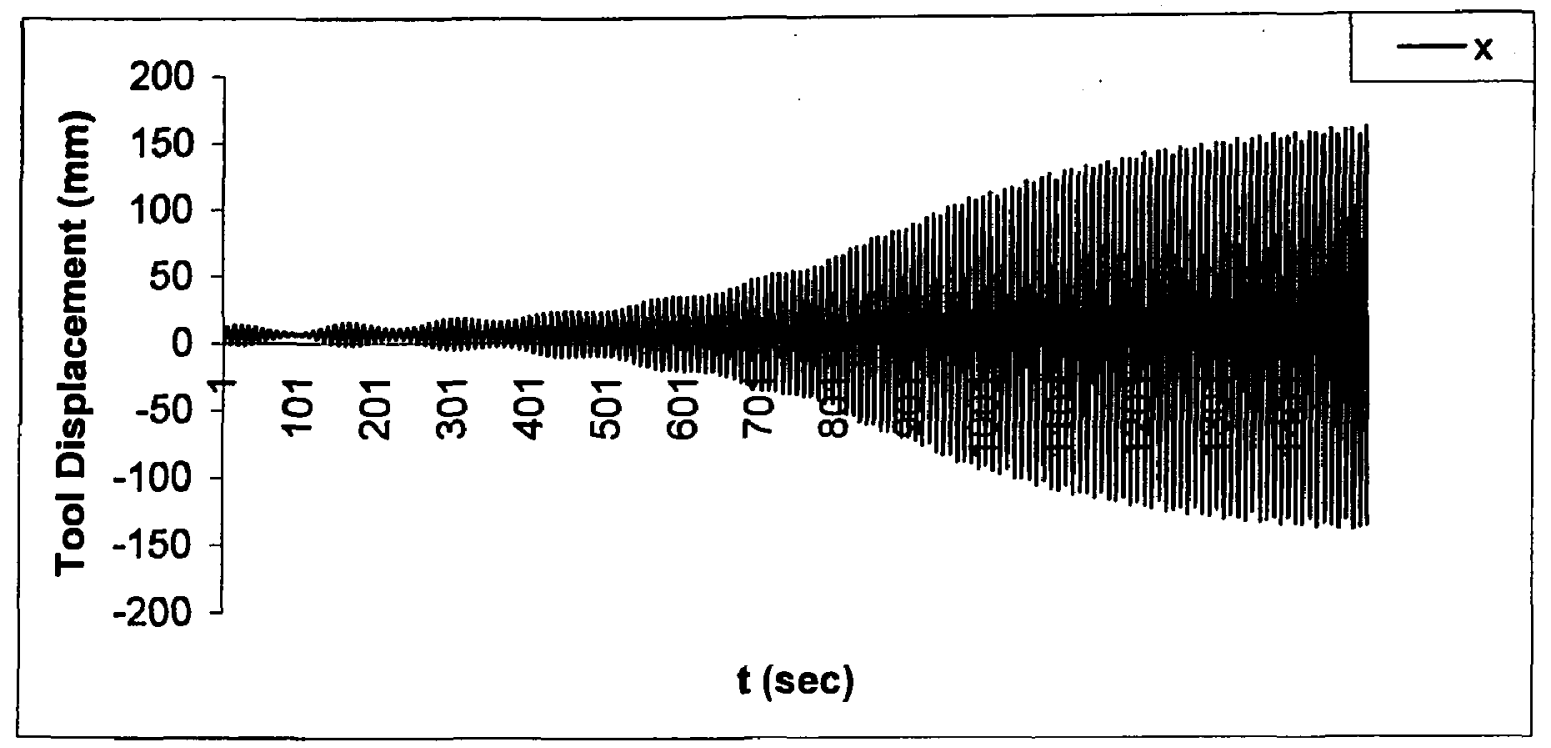

Figure 4.7: Turning Chatter Simulation for $b=3.06 \mathrm{~mm}$

The amplitude of vibration in figure 4.7 is growing larger than average chip thickness. After about 11 revolutions the amplitude reached about $150 \mathrm{~mm}$ and did not grow any more. The reason is that for parts of the vibrational cycles the tool is moving in the air and no longer generates self-excitation energy.

\subsection{Chatter Simulation in Milling}

Milling is a basic machining process by which a surface is generated by progressive chip removal. The cutter is multi-tooth, unlike turning and boring tools (Figure 4.8). Therefore, the number of teeth in milling cutter must be considered for material removal rates (more teeth means more material is removed during the rotation of the cutter). In milling the phasing of undulations produced by subsequent cutter teeth may sometimes have a significant effect on stability [5]. 


\section{END MILLING}

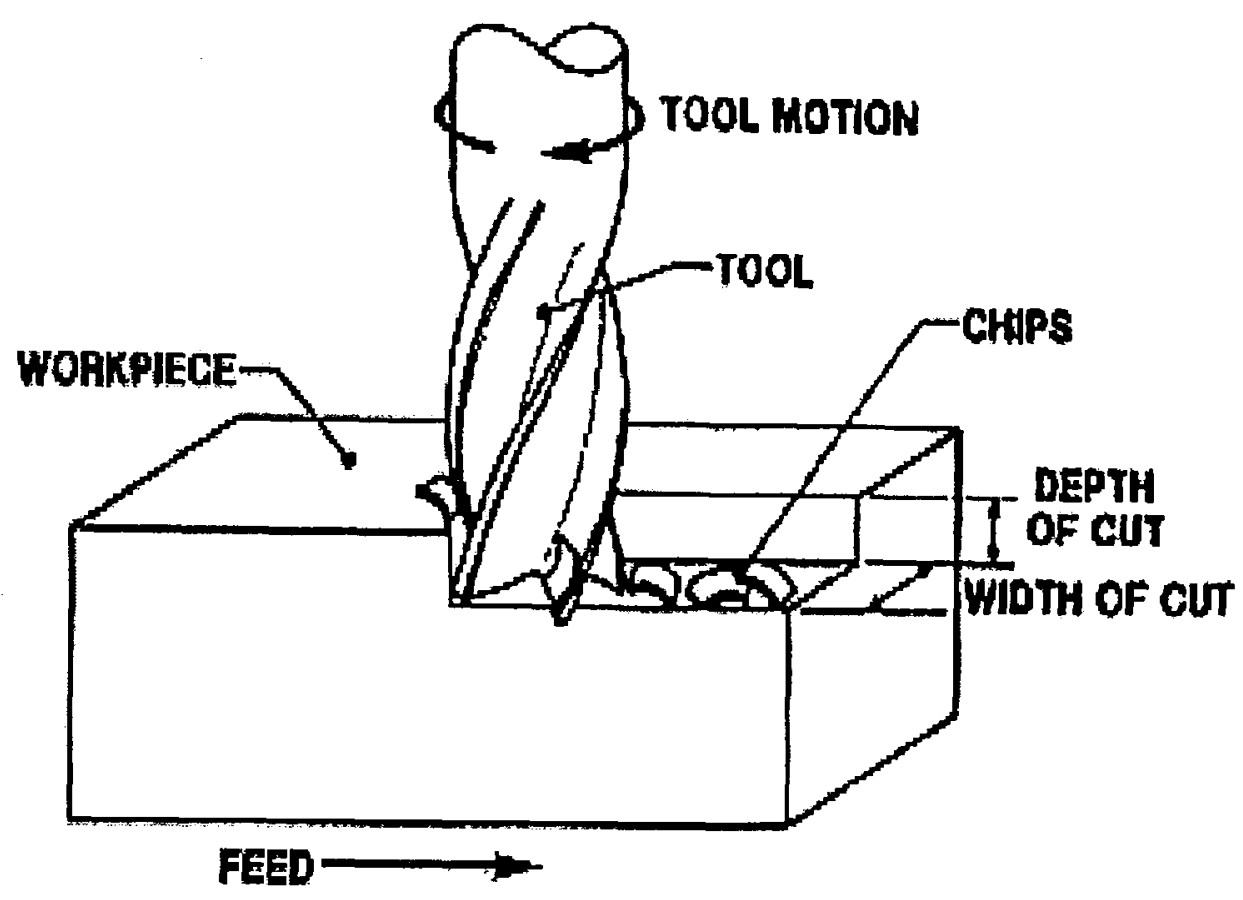

Figure 4.8: End Milling Process [ 25]

\subsection{Simulation Model for Milling}

Chatter in milling is more complex than in turning because the system of forces on the individual teeth rotates with respect to the directions of flexibilities of the vibratory system. Figure 4.9 shows a milling cutter with 8 teeth in a slotting cut, that is, with each tooth cutting through a $180^{\circ}$ engagement. There are 4 teeth cutting simultaneously. The spindle is mounted in a housing with one vibrational degree of freedom in each of the structural axes $X$ and $Y$. the tool rotates with $\mathrm{N}$ rpm, and the work piece feeds with feed rate $f$, chip load $\mathrm{c}$ per tooth in the direction $-X$ 


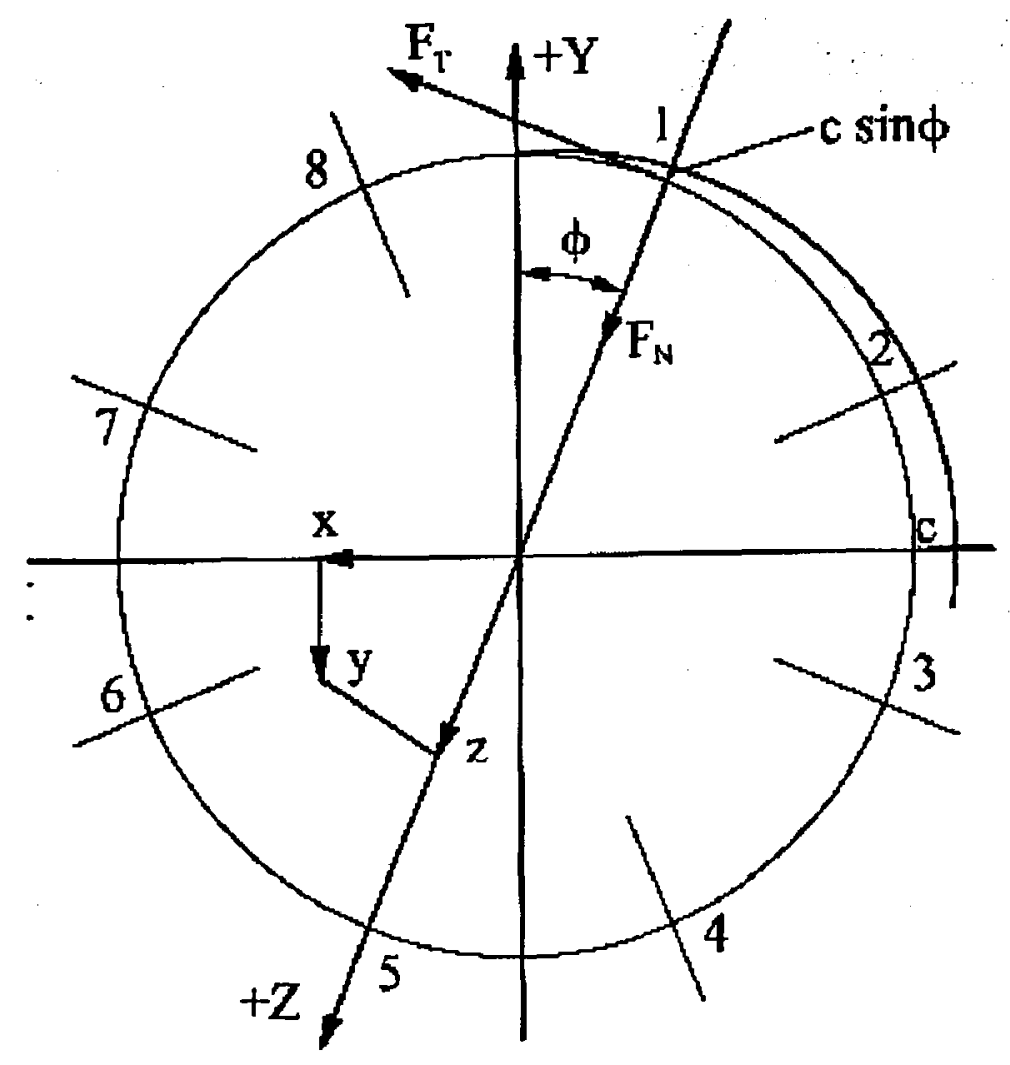

Figure 4.9: Milling cutter with 8 teeth in a slotting cut [5]

A tooth at the angular position $\phi$ produce a tangential force $F_{T}$ and normal force $F_{N}$. The force $\mathrm{F}_{\mathrm{T}}$ is proportional to the chip cross section:

$$
\mathrm{F}_{\mathrm{T}}=\mathrm{k}_{\mathrm{s}} \mathrm{bh}
$$

Where $b$ is the axial width of cut, and

$$
\mathrm{h}=\mathrm{c} \sin \phi-\mathrm{z}(\mathrm{t})+(\mathrm{t}-\mathrm{T})
$$

where $z(t)$ is the current instantaneous vibrational displacement of the cutting edge in the radial direction and $z(t-T)$ is the displacement of the preceding tooth, when it was at the current position, that is, at the time $T$ back, where $T$ is the period of the passage of the teeth , $\mathrm{m}=8$ is the number of teeth and $\mathrm{N}$ is the spindle speed. The displacement $\mathrm{z}$ results from displacement $x$ and $y$

$$
z=x \sin \phi-y \cos \phi
$$


the simulation runs in time steps $\mathrm{dt}$, with 40 steps per tooth and 320 steps per revolution, the cutter rotates by $\mathrm{d} f_{\mathrm{i}}=\pi / 160$. In the first pass the radial displacement $\mathrm{z}$ on all 4 teeth and instantaneous chip thickness $h$ are established. The forces $F 1$ through F4 are obtained while a check is made whether a tooth really cuts at a particular instant, i.e., $h>0, F>0$ or whether it is jumping out of the cut, i.e.,

$\mathrm{h}<0, \mathrm{~F}=0$. The component forces Fx and Fy are obtained and accelerations $\ddot{x}$ and $\ddot{y}$ calculated and integrated into velocities and further integrated into the next displacements $x(n+1), y(n+1)$. The simulation continues through time steps.

The degree of stability of a given structure, exhibiting modes of vibration between the tool and work piece, can be expressed by the value of the limit width of chip, $b_{\text {lim, }}$ at which chatter just starts to occur. In order to get estimate of the chatter limit $b_{\text {lim }}$, the rotating system is represented by the stationary mean position of the middle tooth, that is, at $\phi=$ $90^{\circ}$. Using specific force, $\mathrm{Ks}=1500 \mathrm{~N} / \mathrm{mm}^{2}$, in simplified "mean" stationary system in which $\mathrm{Y}$ mode does not participate [5]. Since,

$$
\mathrm{b}_{\lim }=\frac{4 k \times \xi \times 2}{2 \mu_{x} \times K_{s} m}
$$

From equation (4.21) values of $b_{\text {lim }}$ can be expressed as;

$$
b_{\lim }=\frac{4 k \times \xi \times 2}{2 \mu_{x} \times K_{s} m}=1.09 * 10^{-3}=1.01 \mathrm{~mm}
$$

Simulation results for the set of model parameters in Table 4.2 are shown in Figure 4.9. 


\begin{tabular}{|l|l|}
\hline $\mathrm{k}_{\mathrm{x}}$ & $7.0^{*} 10^{7} \mathrm{~N} / \mathrm{m}$ \\
\hline $\mathrm{k}_{\mathrm{y}}$ & $1.0^{*} 10^{8} \mathrm{~N} / \mathrm{m}$ \\
\hline $\mathrm{m}$ & $100 \mathrm{~kg}$ \\
\hline $\mathrm{u}_{\mathrm{x}}$ & $70^{0}$ \\
\hline $\mathrm{u}_{\mathrm{y}}$ & $0^{0}$ \\
\hline$K_{\mathrm{s}}$ & $1500 \mathrm{~N} / \mathrm{mm}^{2}$ \\
\hline $\mathrm{c}_{\mathrm{x}}$ & $535 \mathrm{~N} /(\mathrm{m} / \mathrm{s})$ \\
\hline $\mathrm{c}_{\mathrm{y}}$ & $737 \mathrm{~N} /(\mathrm{m} / \mathrm{sec})$ \\
\hline$\phi$ & $90^{0}$ \\
\hline $\mathrm{N}$ & $3000 \mathrm{rpm}$ \\
\hline$\zeta_{\mathrm{x}}$ & 0.016 \\
\hline$\zeta_{\mathrm{y}}$ & 0.018 \\
\hline
\end{tabular}

Table 4.2: Model Parameters for Milling

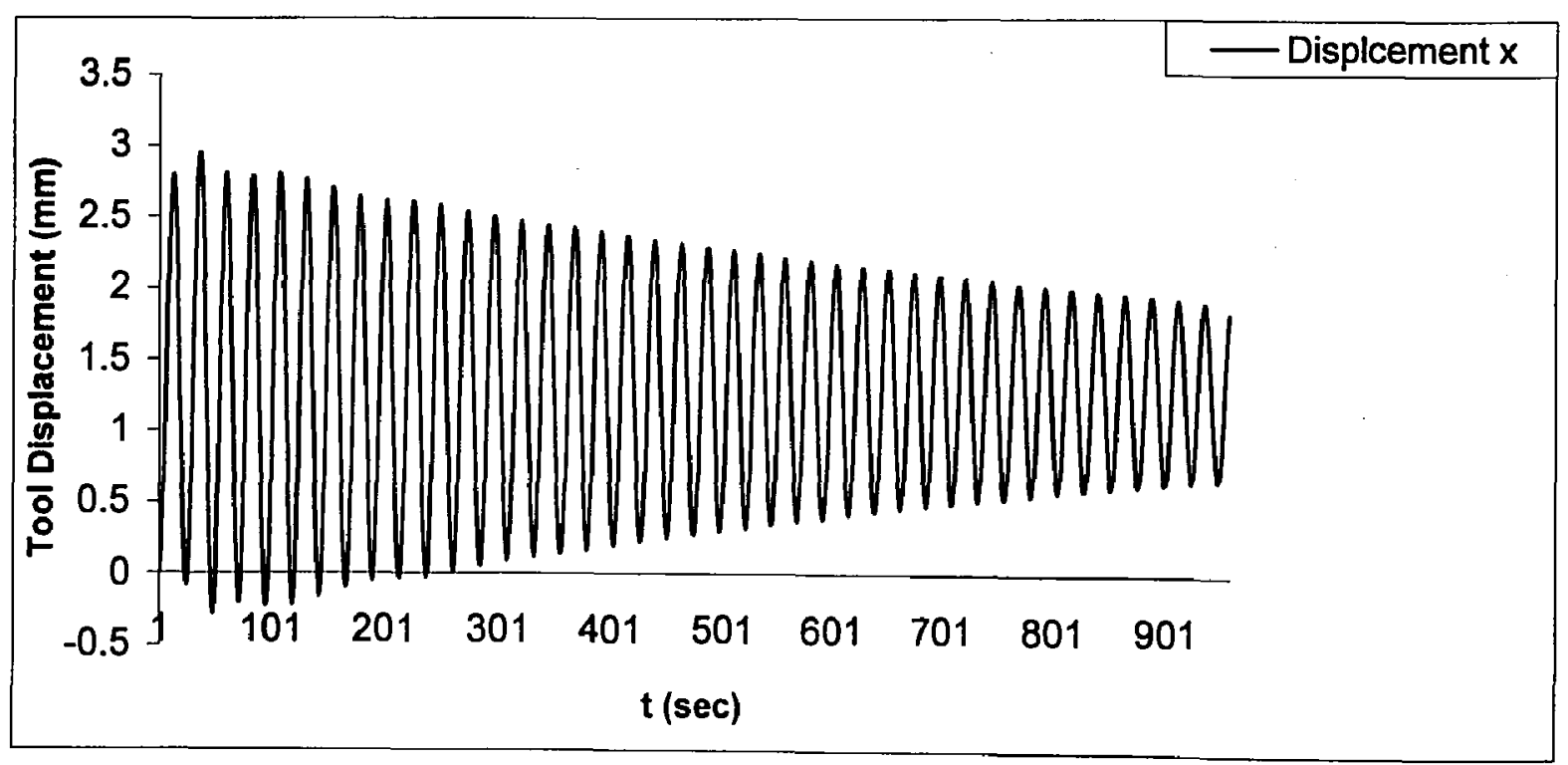

Figure 4.9(a): Milling Chatter Simulation for $b=1.00 \mathrm{~mm}$ 


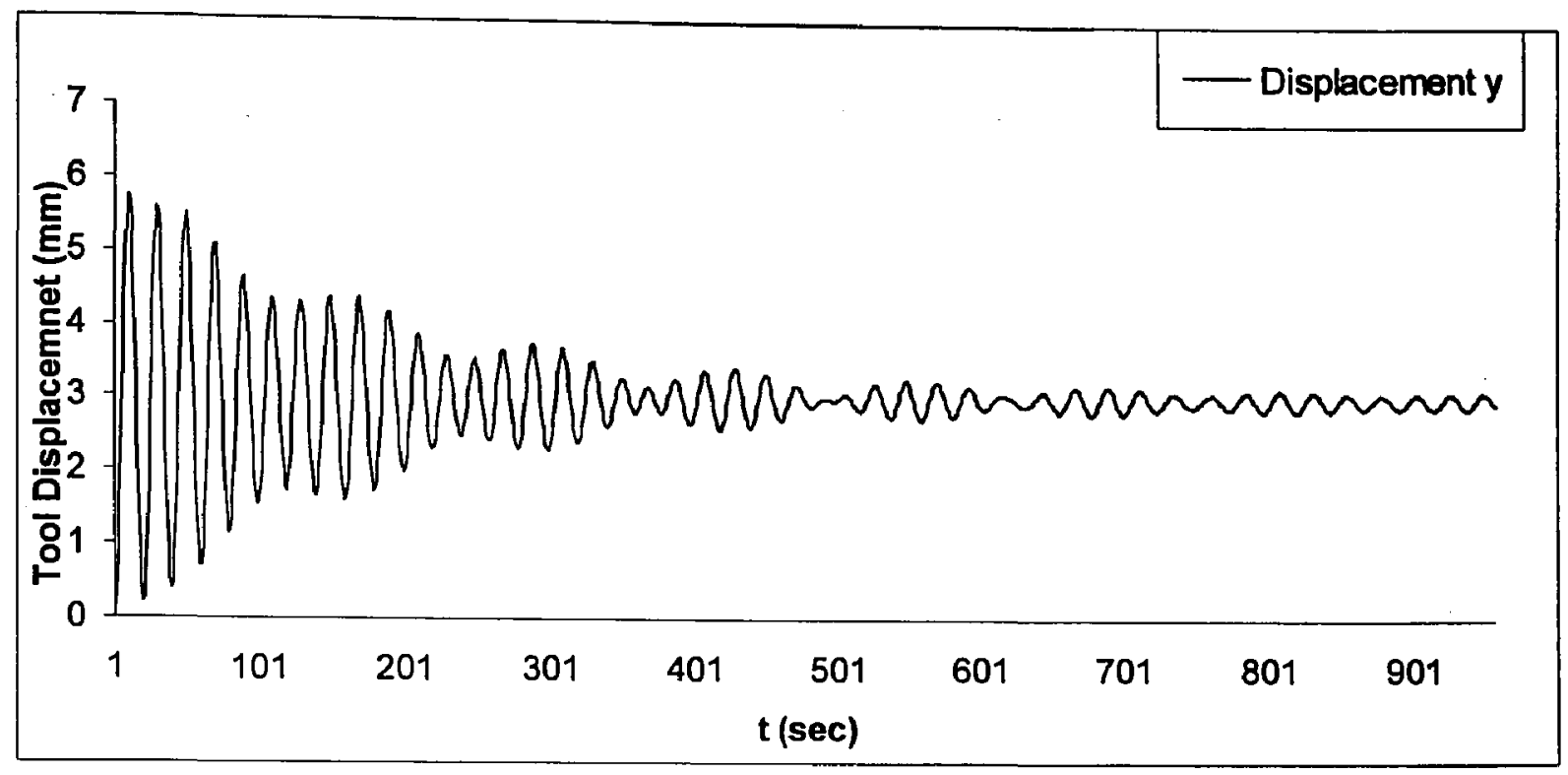

Figure 4.9 (b): Milling Chatter Simulation for $b=1.00 \mathrm{~mm}$

Figures 4.9 (a) and 4.9 (b) show the simulation results for $b=1.00 \mathrm{~mm}$. In this case, the vibrations are decreasing revolution after revolution. And after a great number of revolutions they would develop a constant amplitude. In the y displacement, the magnitude of vibrations during first two revolutions is, on average, about $5 \mathrm{~mm}$ peak to peak (PTP). This case is stable and no suppression is needed.

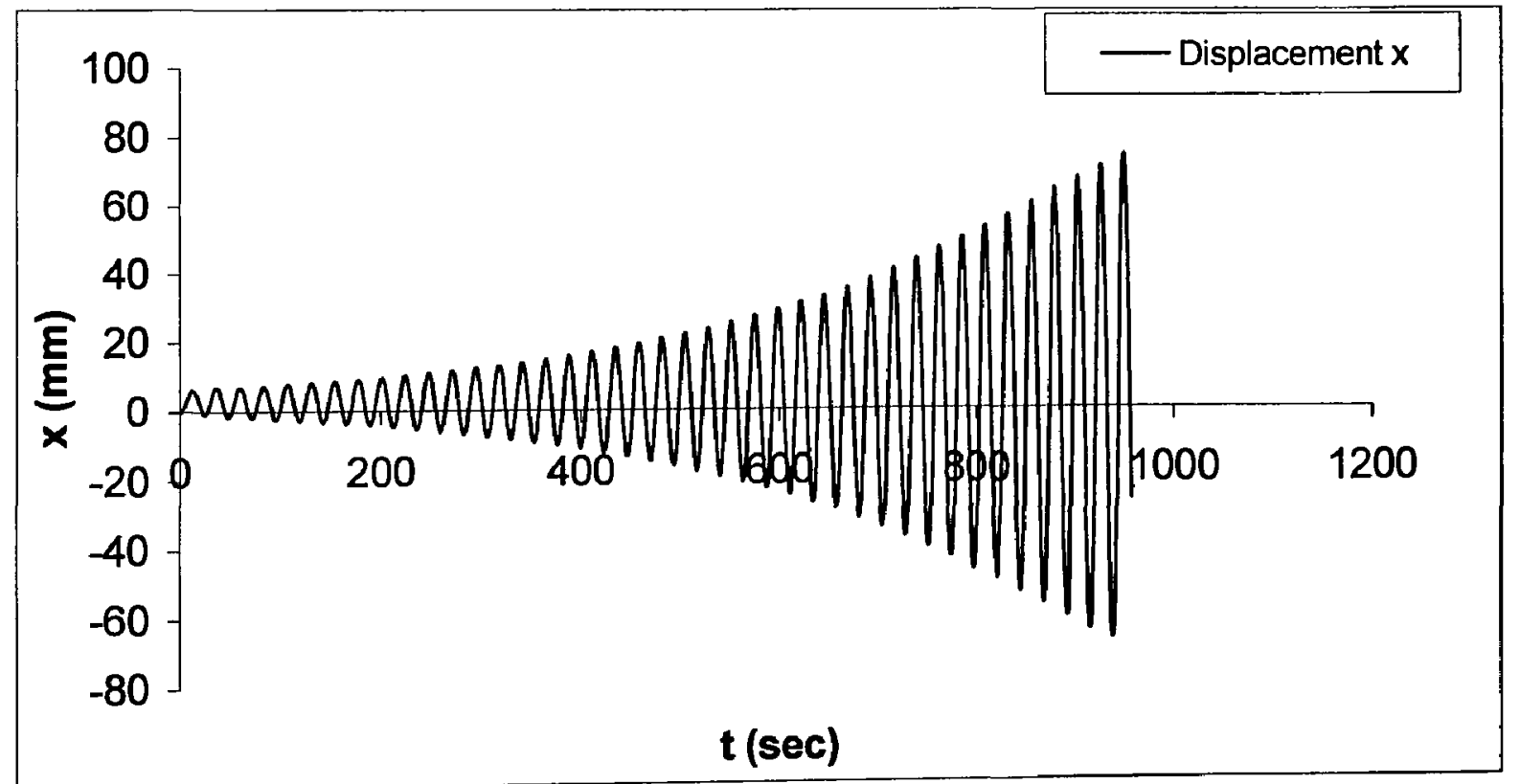

Figure 4.10(a) : Milling Chatter Simulation for $b=2.00 \mathrm{~mm}$ 


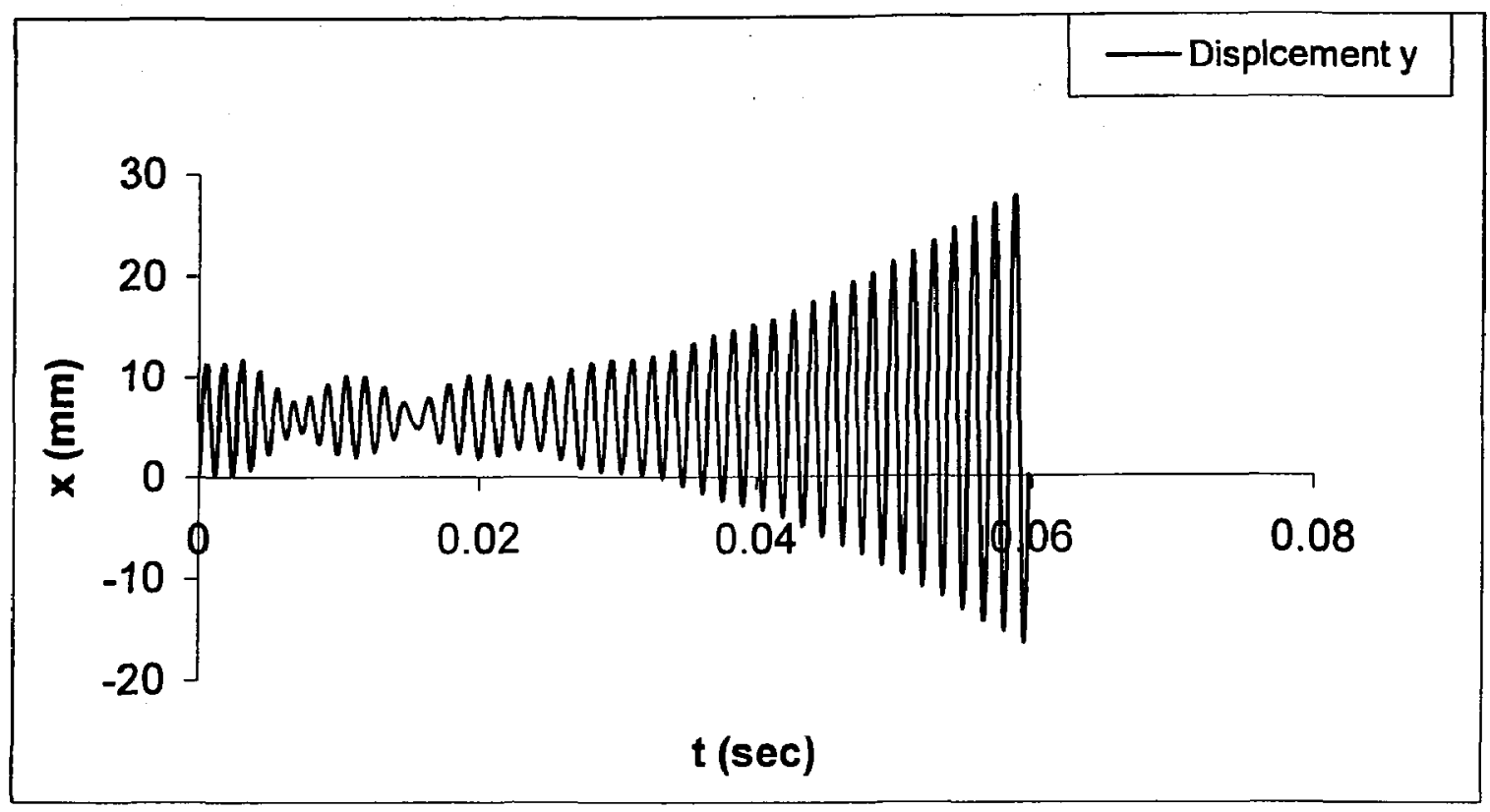

Figure 4.10 (b): Milling Chatter Simulation for $b=2.00 \mathrm{~mm}$

Figures 4.10 (a) and 4.10 (b) show the simulation results for $b=2.00 \mathrm{~mm}$ in $\mathrm{x}$ and $\mathrm{y}$ displacement. In this case, the growth of vibrations is very fast. The amplitude of vibrations is growing larger than the average chip thickness. After $0.06 \mathrm{sec}$ it has reached almost $25 \mathrm{~mm}$. This is the direct result of non-linearity of self- excited vibrations caused by the fact that as vibration grows larger, the tool loses contact with the work piece. 


\section{Chapter 5}

\section{Results}

\section{$5.1 \quad$ Introduction}

The methodology described in Chapter 3 is evaluated through simulation studies. The results of training and testing numerous neural network architectures are presented in this chapter.

\subsection{Chatter Suppression in Turning}

As described in Chapter 3, a multilayer feed forward neural network is considered for the task of modeling the dynamics of cutting process including time delays between signals. The number of input and output nodes and hidden nodes describes the architecture of such a neural network. The input and output node counts are determined by the model requirements while the number of hidden layers and nodes in each layer are determined by model performance. The model performance is also affected by the design of the training sets, data preparation and presentation to the neural network.

\subsubsection{Neural Network Architecture}

In order to train the neural network for turning, necessary training sets must be assembled. Figure 5.1 shows the neural model used in turning. The network has 11 inputs and 5 outputs. The first set of inputs are the frequency response at the start of the sampling period characterized by $\mathrm{p}$ coefficients, where $\mathrm{p}$ is the order of AR model described in Chapter 3. For the case of modeling the frequency response of the turning process $\mathrm{p}=3$ was found to be adequate. It was also found that the scaling factor XMS improves the optimization process so it was added to the neural network. 


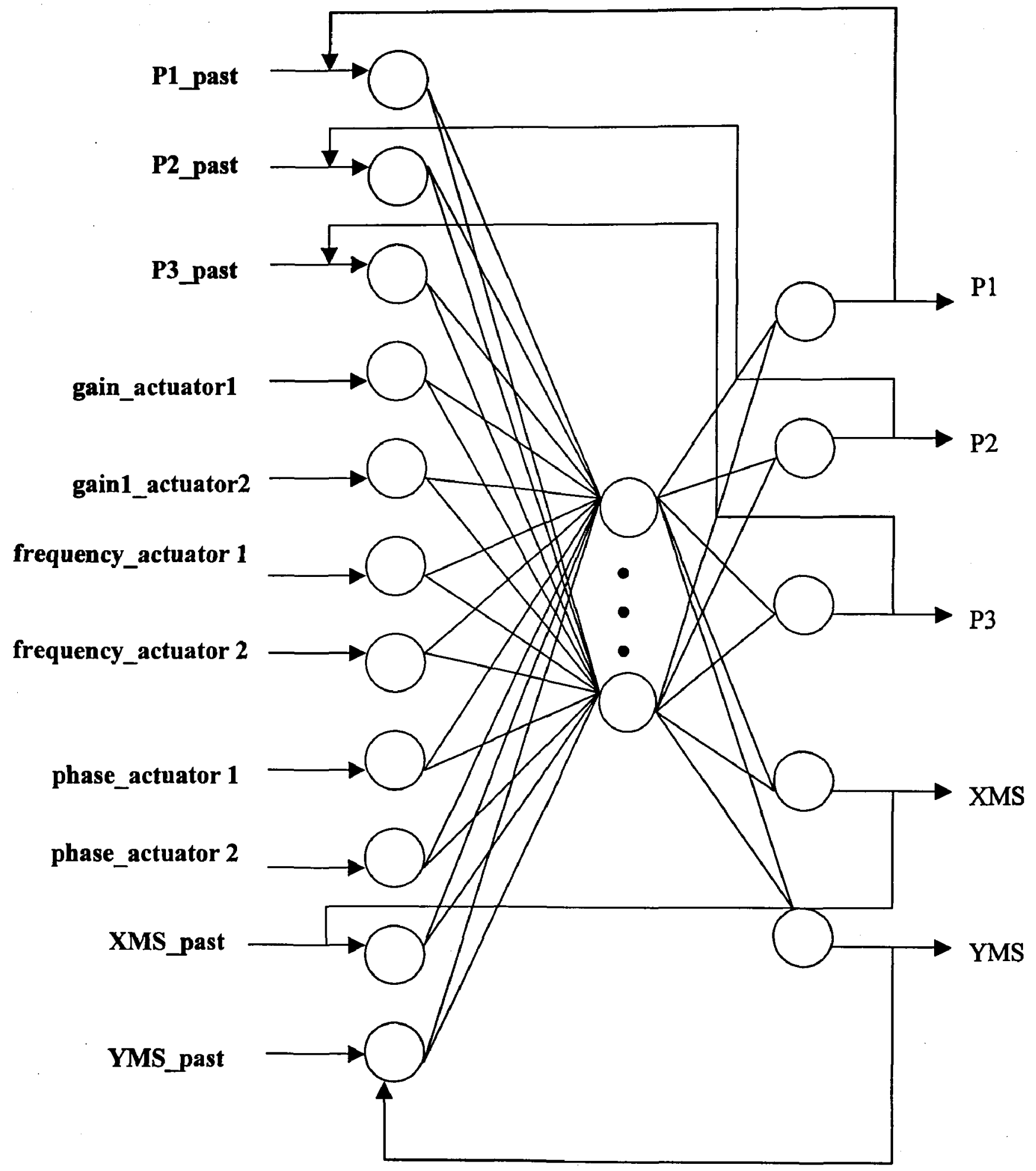

Figure 5.1: Neural Network Model for Turning 
The next sets of inputs are actuator parameters (Figure 5.2). These include gain, frequency and phase delay.

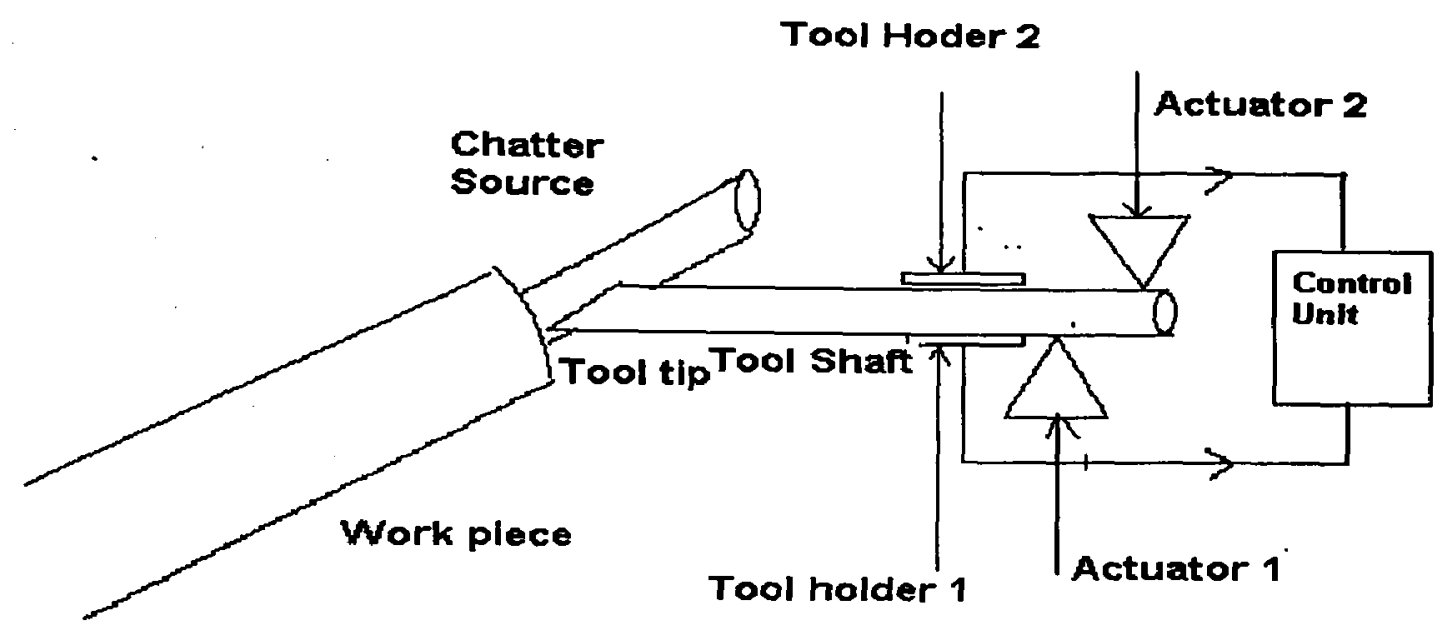

Figure 5.2: Simplified Cutting Process with Two Actuators

The last input is the maximum amplitude of the vibration in the sampling period Ymax (Figure5.3).

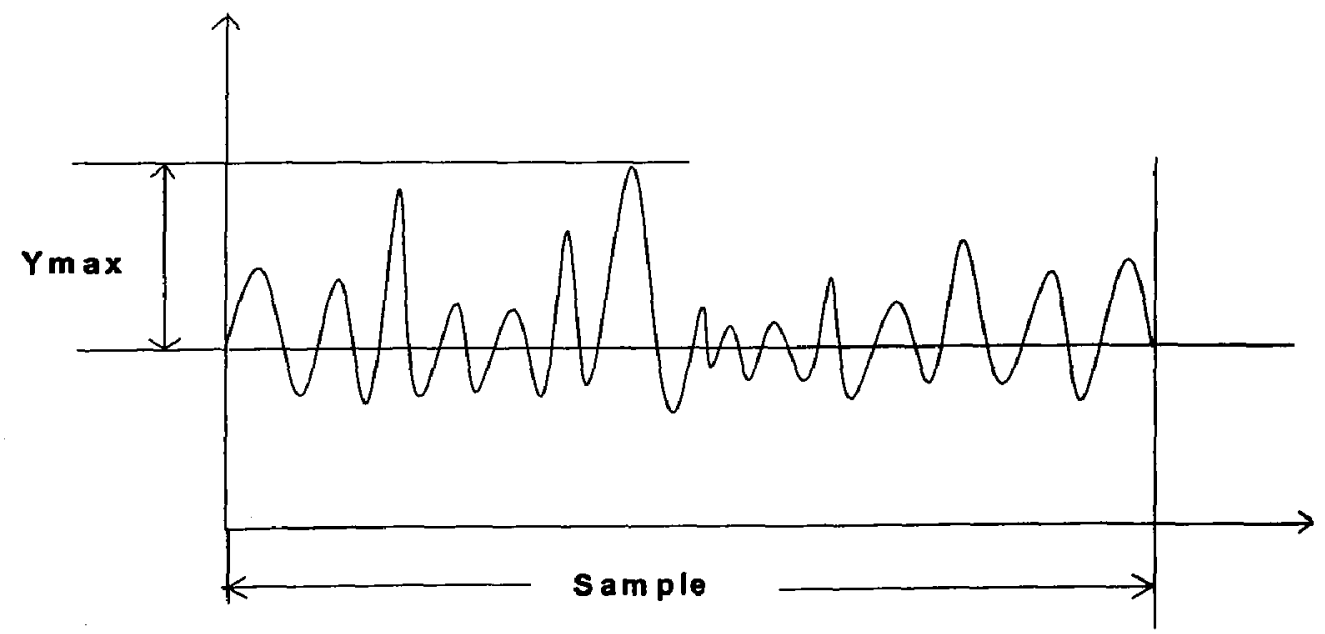

Figure 5.3: Sampling Period with Maximum Amplitude 
The outputs of the neural network model are predicted estimates of the frequency response of the next sampling period represented by $\mathrm{p}$ coefficients and XMS and predicted maximum amplitude of the next sampling period.

\subsubsection{Off -Line Training}

The output of the sigmoid function is limited to values between $[1,0]$ (Figure 5.4).

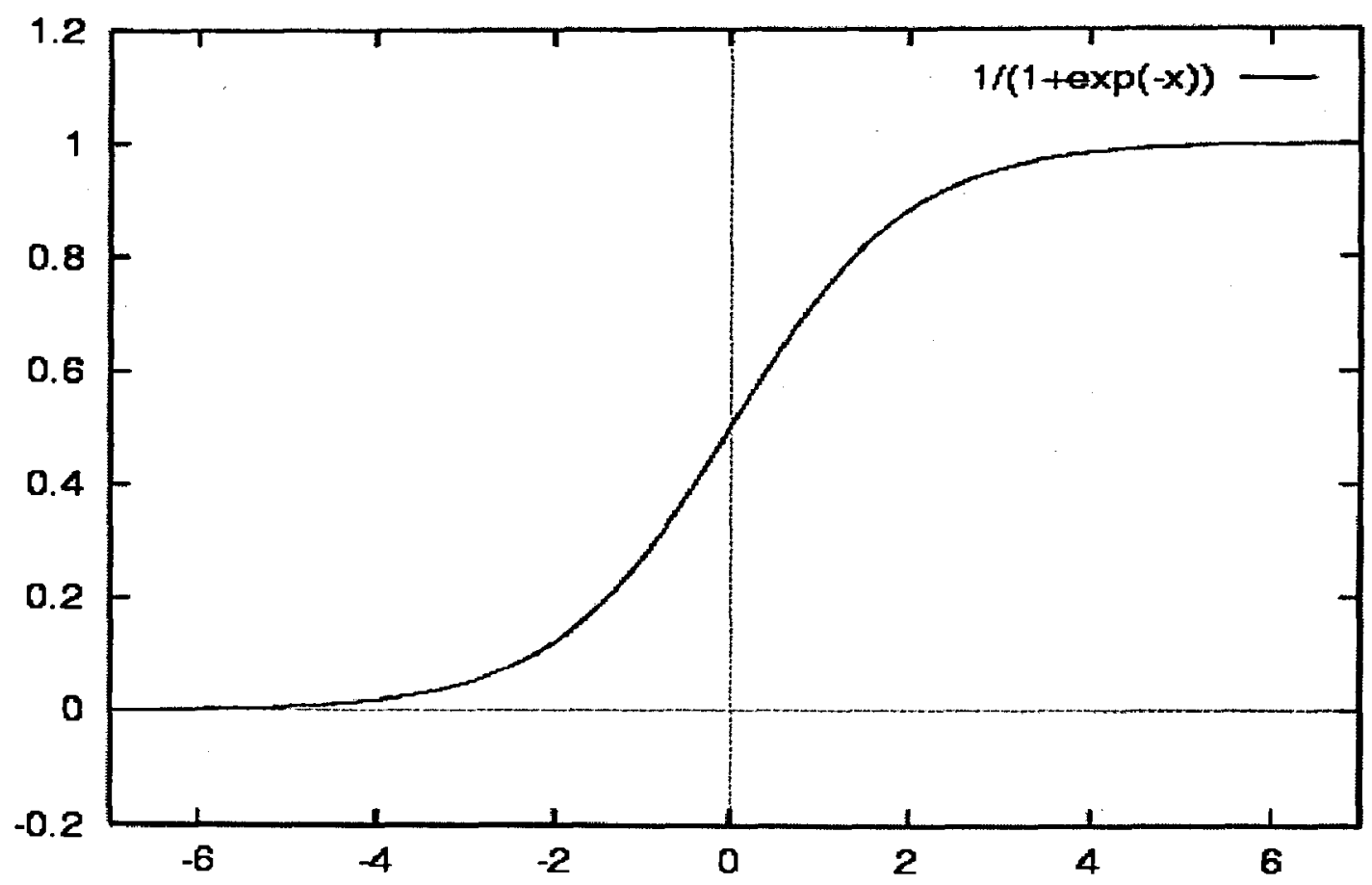

Figure 5.4: Sigmoid Function

Although the input range is not limited, it can also be normalized to facilitate the training process. Limits between 0.1 and 0.9 are used for normalizing data as it reduce the effects of the asymptotes. The simplest way to scale outputs is by using a linear scaling formula, i.e.,

$$
\mathrm{V}_{\text {scaled }}=\frac{0.8\left(V-V_{\min }\right)}{V_{\max }-V_{\min }}+0.1
$$


Where $\mathrm{V}$ is the variable to be scaled, $\mathrm{Vmax} \& \mathrm{Vmin}$ are maximum and minimum values. Scaling of all the data assures that all have the same range, and therefore training will proceed will proceed faster.

\subsubsection{Training and Test Set}

The performance of the neural network model is evaluated using the root mean square (RMS) error. Each neural model is evaluated twice: once against the training set consisting of patterns with which the neural network was trained and once with the test set. A computer program was written in the $C$ language to generate data. The test set consists of data set aside for evaluating the generalization capability of the neural model. The neural network model has not seen these patterns during training.

In each case, the total error over the entire set is calculated, i.e.,

$$
\sum_{j=0}^{j=s} \sqrt{\frac{1}{r}} \sum_{i=0}^{r-1}\left(t_{i}-a_{i}\right)^{2}
$$

RMS error $=$

s

Where, $\mathrm{s}$ is the number of output variables in the network.

Initial training was performed by repeatedly presenting the training set to the network until a minimum training error was reached. Once a minimum training error was reached the network was presented with a test set. In order to prevent over training different NN with varying number of hidden nodes were tested. The results are shown in Figure 5.5. 


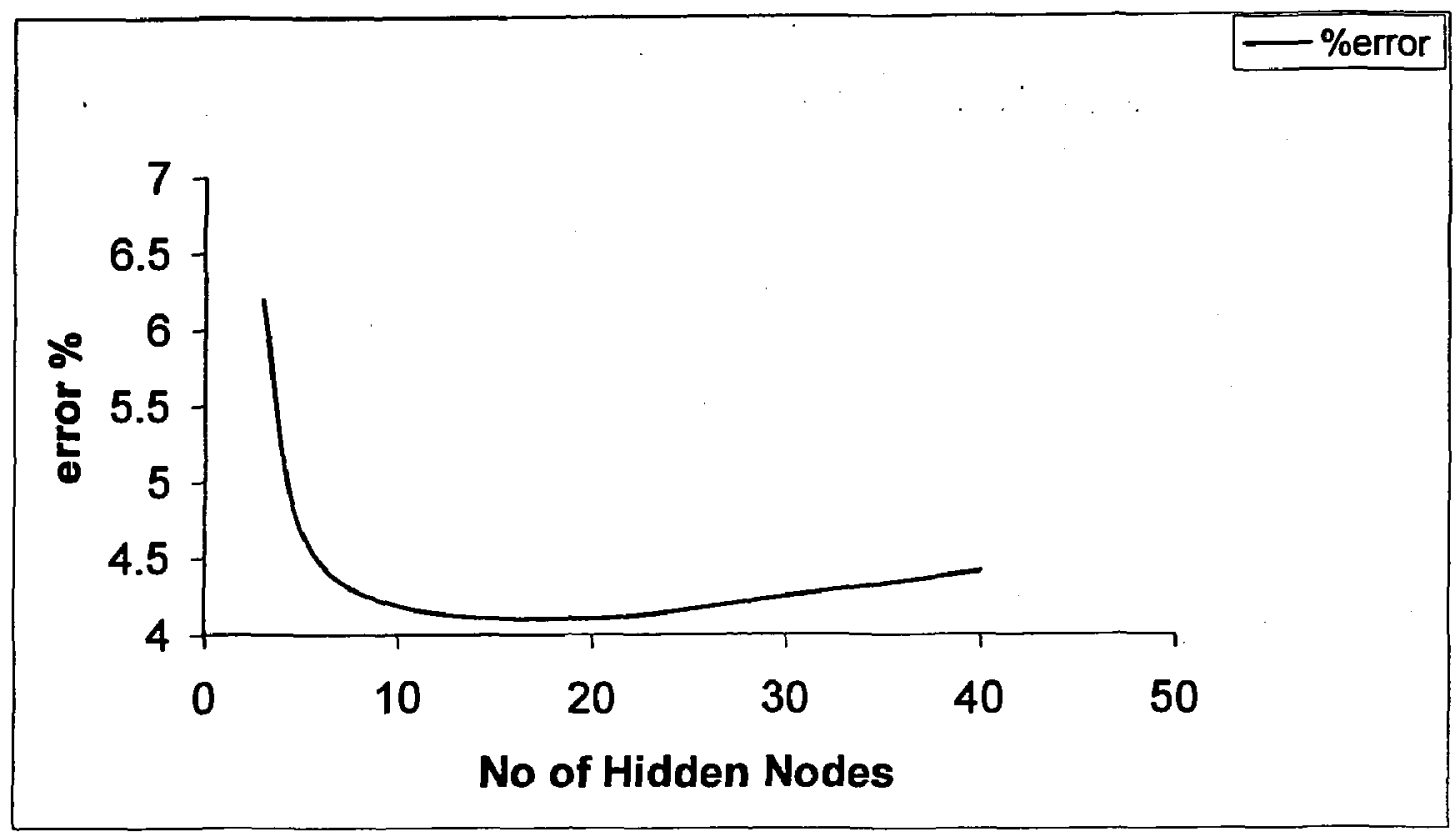

Figure 5.5: Neural Network with Varying Number of Hidden Nodes

The training error reduces continually as the number hidden nodes increases. The test error, however, increase with the addition of hidden nodes. This indicates that for NN with more than 20 hidden nodes, the NN starts to over train and therefore loses some of the generalization capability.

\subsubsection{Chatter Suppression System}

After achieving adequate results in off-line training the NN model was used for simulation studies. Figure 5.6 shows the block diagram of the process.

After starting the simulation of the cutting process, if chatter is detected the suppression system will activate. The trigger for detecting the chatter is amplitude of vibration. Once the chatter is detected, the actuators will be activated 


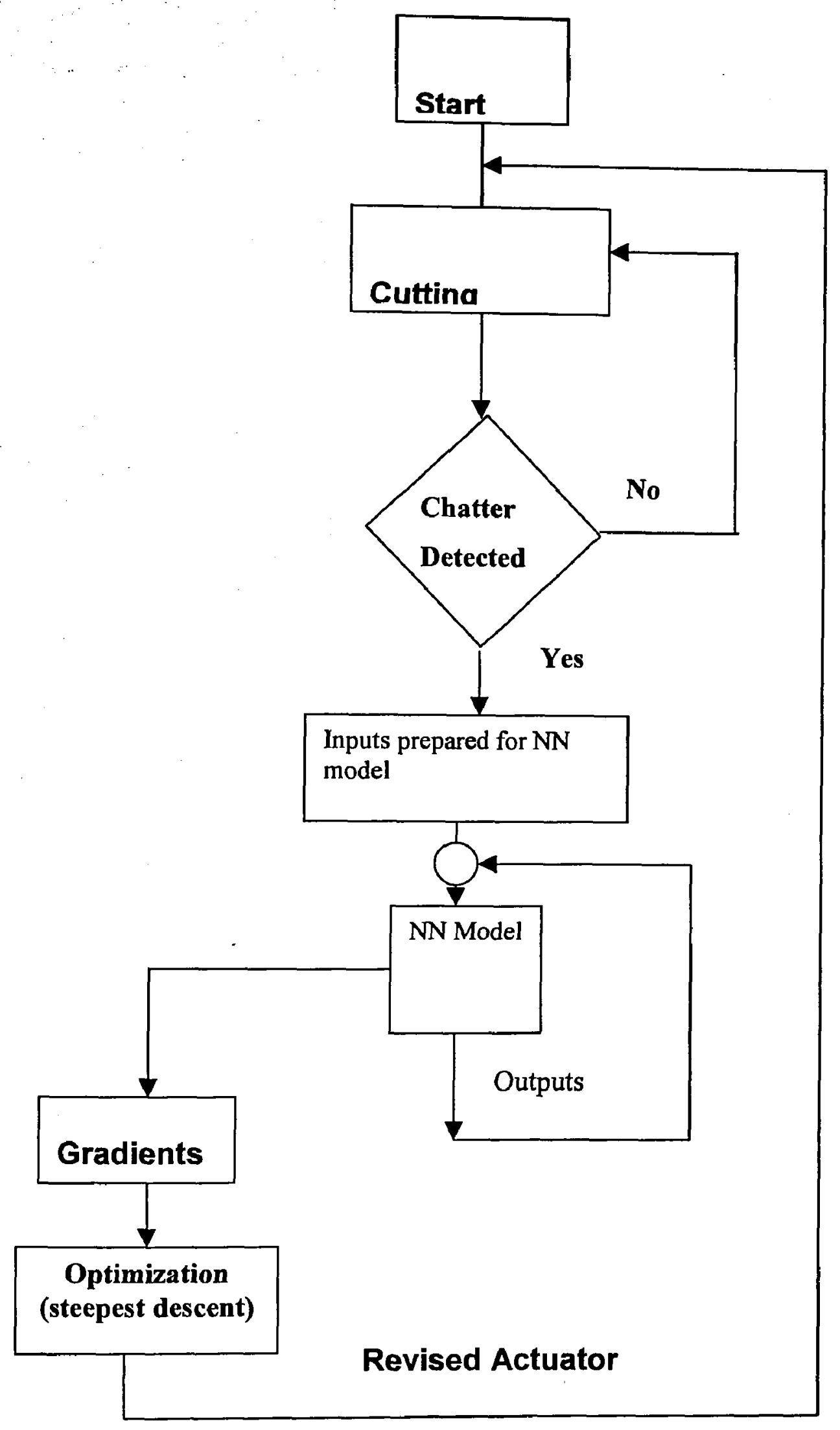

Figure 5.6: Block Diagram of Chatter Suppression System 
In order to determine the parameters of the two actuators (Figure 5.2) the neural network has to generate gradients which will be used to optimize the actuator parameters setting. The tapped delay lines between inputs and outputs of the neural network model will ensures the capability of the neural model to capture the dynamics of the entire system. The optimization module using steepest descent algorithm will modify the actuators settings until chatter is adequately suppressed.

\subsubsection{Simulation Results}

A simulation result with and without deployment of chatter of the chatter suppression system is shown in Figure5.7 and Figure 5.8.

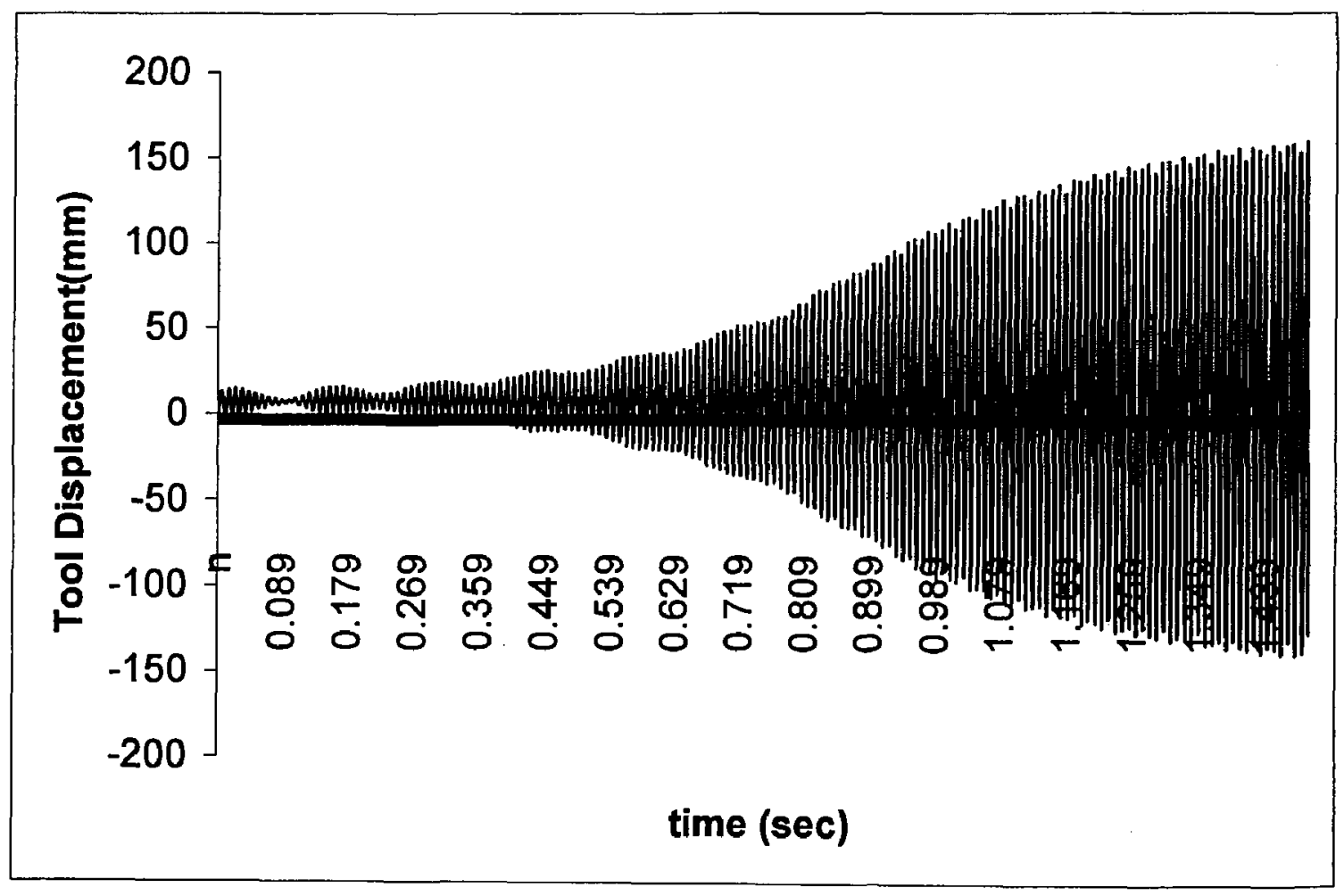

Figure 5.7: Tool chatter with active suppression system Off 


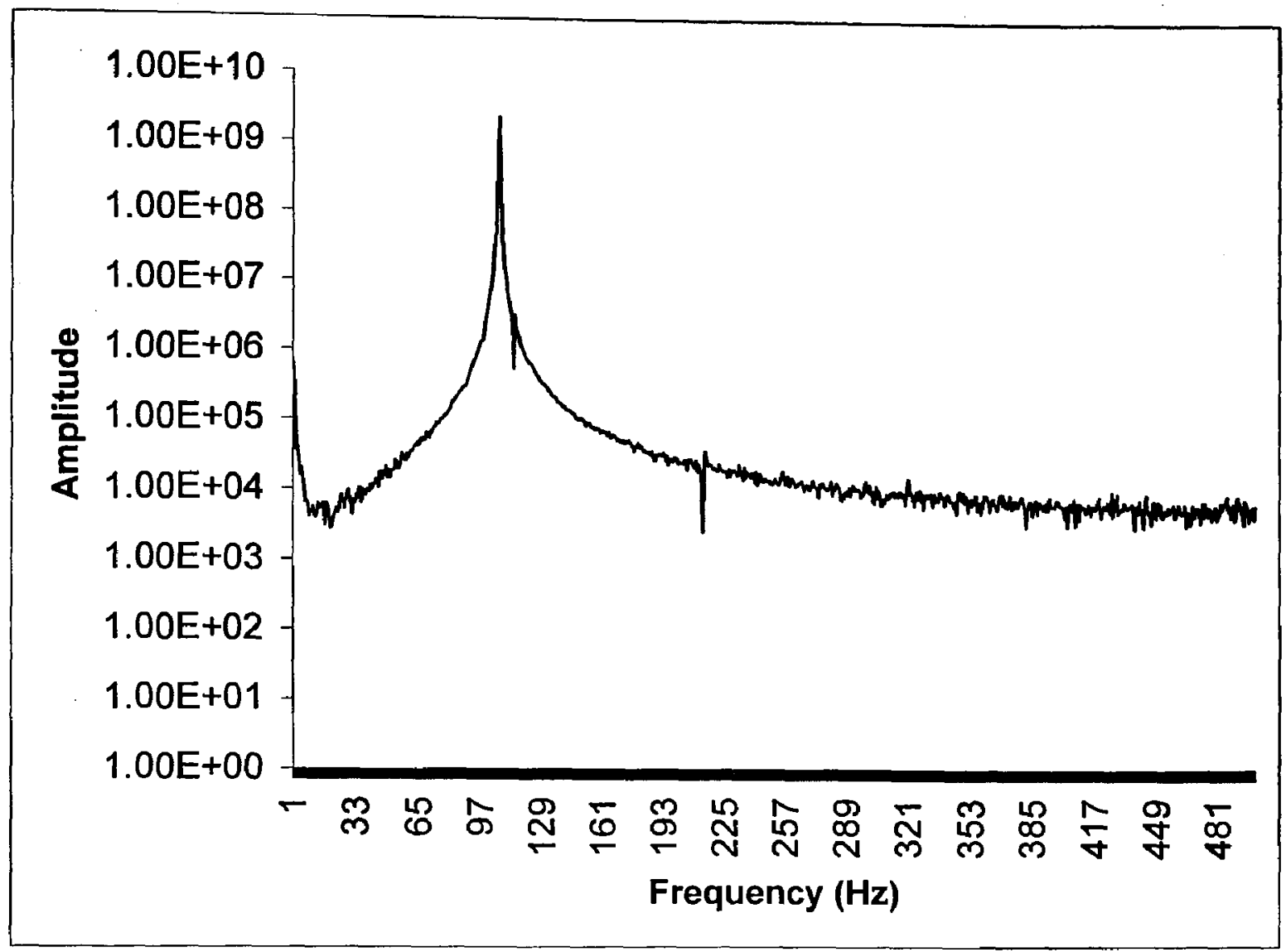

Figure 5.8: Frequency response of the tool with active suppression system off.

Figure 5.7 and Figure 5.8 show the simulation results in time and frequency domain when a critical chip width of $b=3.63 \mathrm{~mm}$ is used and suppression system is off. The growth of vibration is very fast and regenerative chatter mechanism is evident. The vibration is reinforced until a limiting value is reached. This is a direct result of non-linearity of the system.

The non-linearity is caused by the fact that as vibration grow larger, the tool loses the contact with work piece and force is effectively zero. 


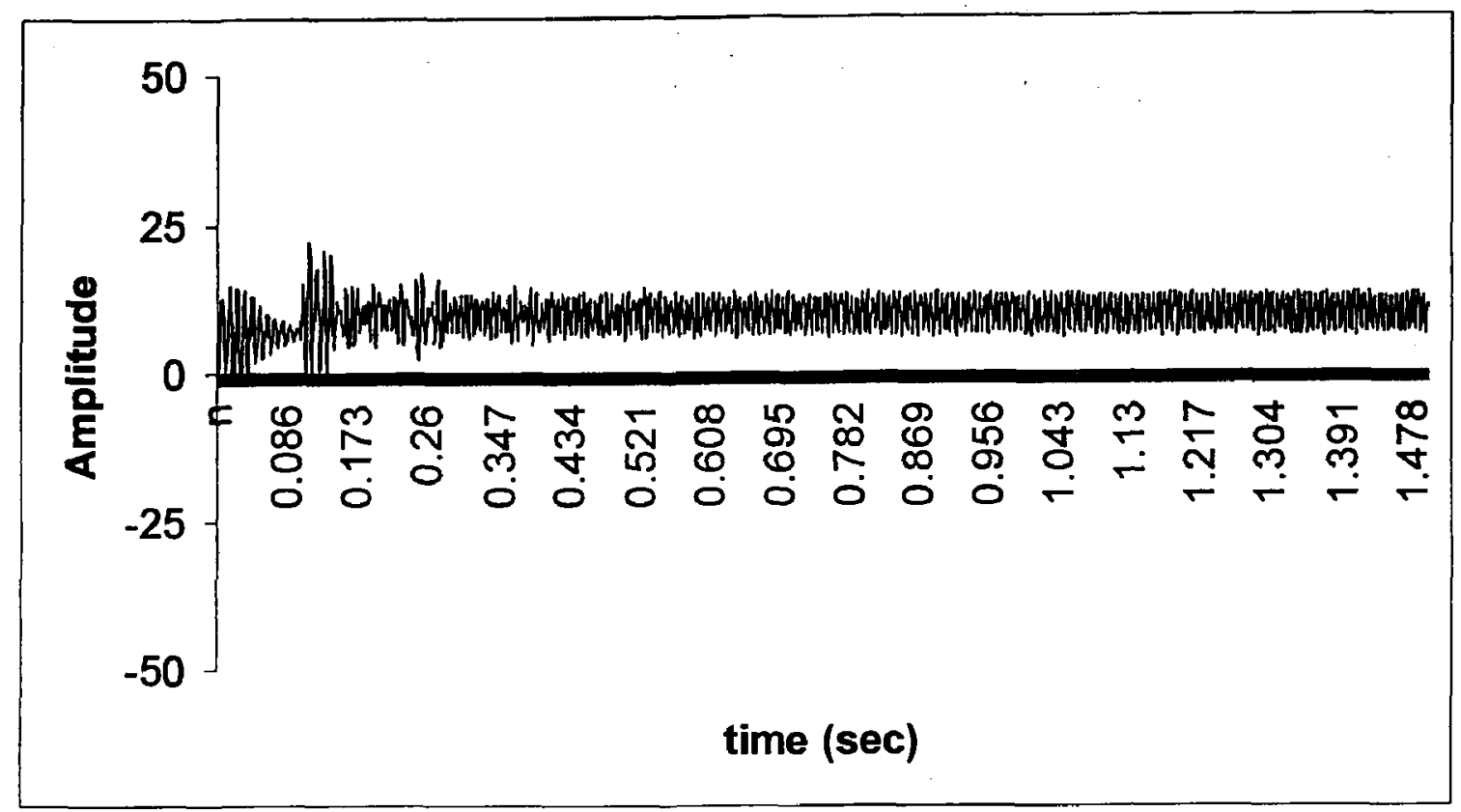

Figure 5.9: Tool vibration with active suppression system On

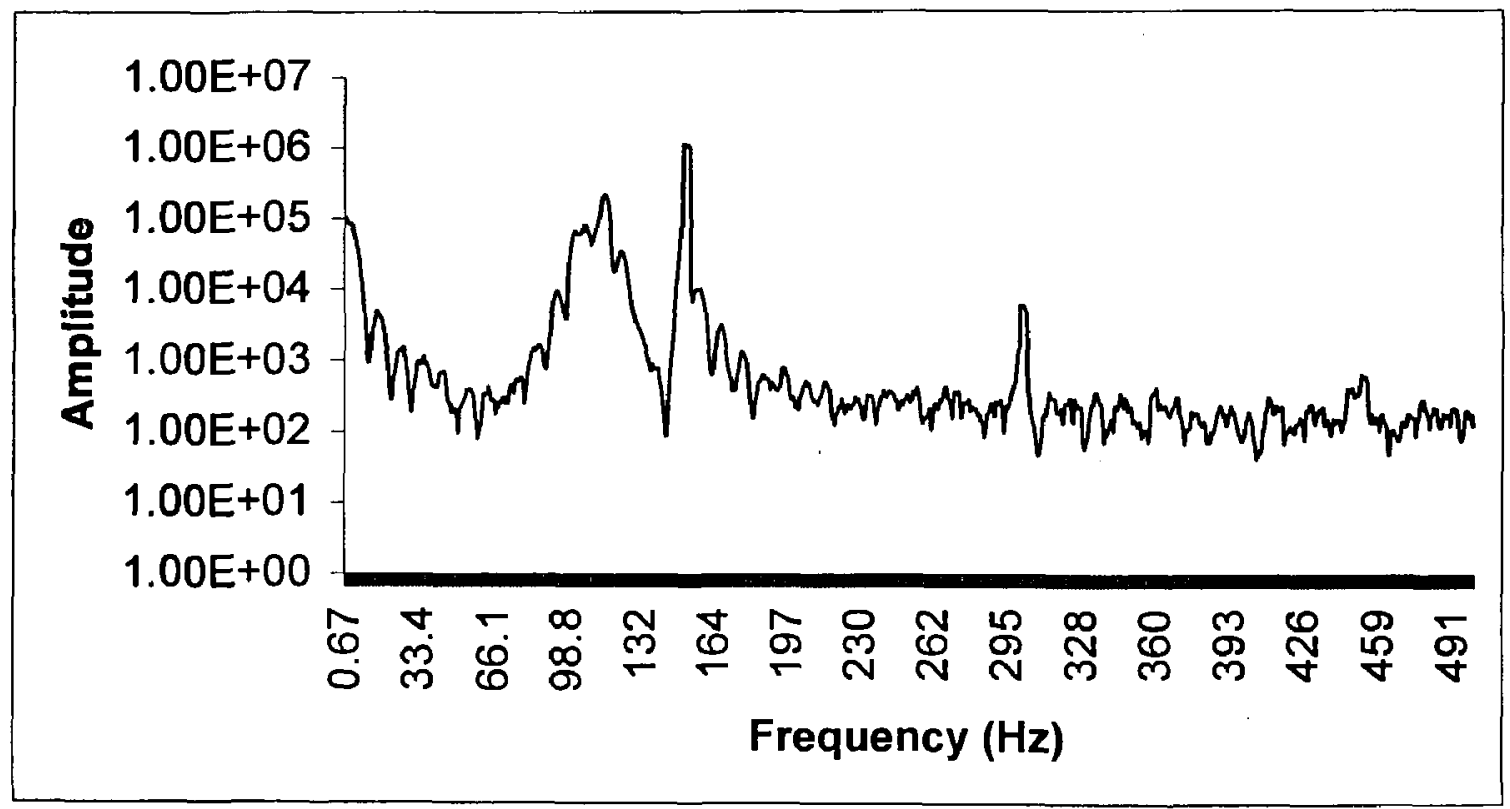

Figure 5.10: Frequency response of the tool with active suppression system On.

Figure 5.9 shows the simulation result when the suppression system is on. The effectiveness of the proposed methodology is shown in this figure, the tool vibration is kept to a minimum. Figure 5.10 shows the frequency response corresponding to this condition 


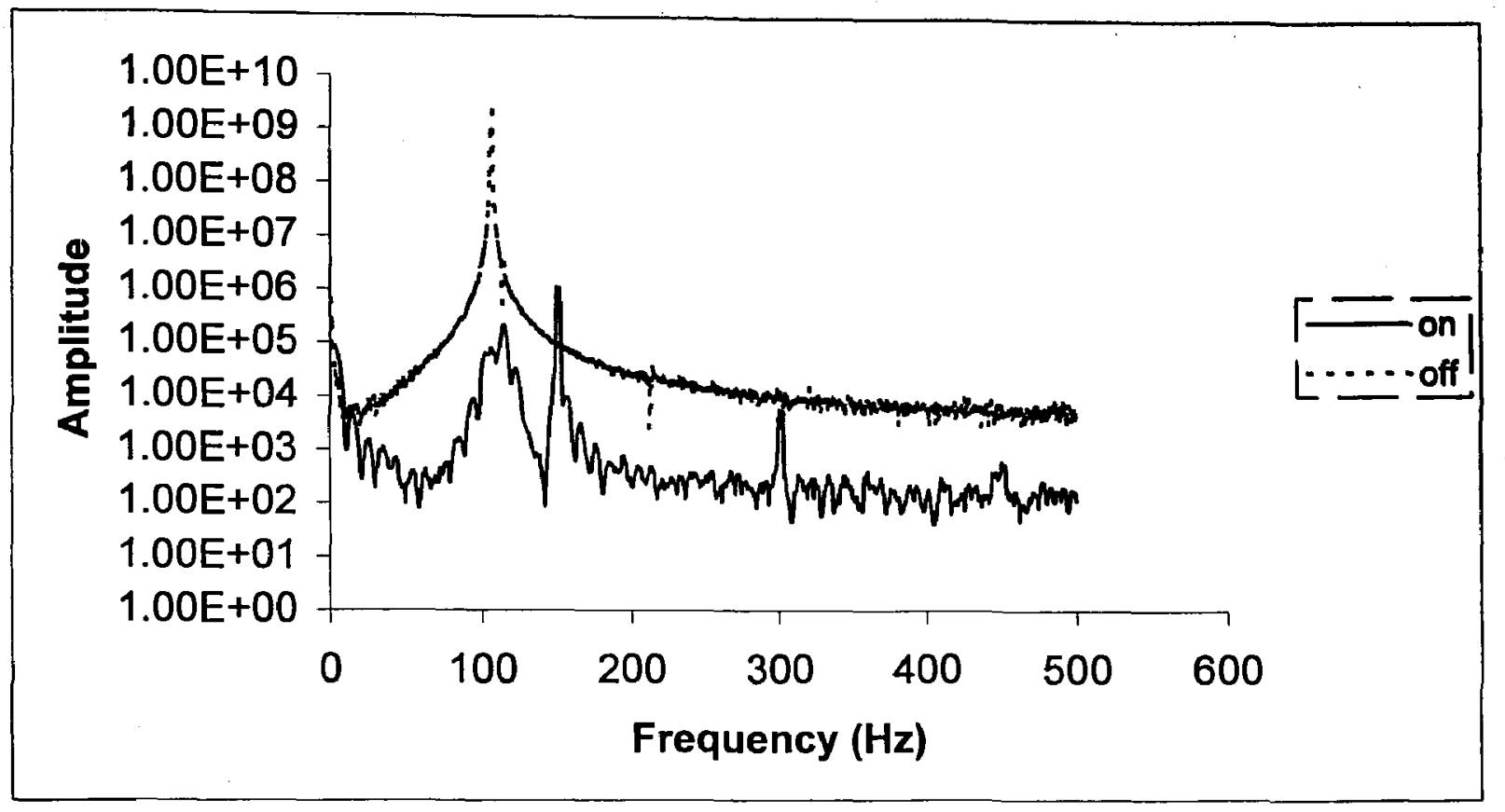

Figure 5.11: Comparison of frequency response of the tool with active suppression On and Off.

Figure 5.11 shows the comparison of frequency response of the overall cutting process with and without active suppression system. It shows two peak when the system is on.

The active chatter suppression system was used to reduce the chatter. The chip width was set to critical value of $b=3.06 \mathrm{~mm}$. Based on these simulation results the proposed methodology seems to be capable of suppressing chatter in turning and boring operations.

\subsection{Chatter Suppression in Milling}

The neural network model for suppression of milling is shown in Figure 5.12. Two actuators are employed along $\mathrm{X}$ and $\mathrm{Y}$-axis of the machine. The parameters of these actuators are the first six input to the neural model. 
Since chatter could happen both in $\mathrm{x}$ and $\mathrm{y}$ direction frequency response of both these axes has to be monitored. All poles method (see section 3.7) with 3 coefficients and scaling factor XMS were used to model these frequency responses. Finally, the maximum amplitudes in $\mathrm{x}$ and $\mathrm{y}$ direction during the current sampling period were included in the input vector. The outputs of the neural model are predicted frequency response and minimum amplitudes in the next sampling period.

The next sets of inputs are actuator parameters (Figure 5.13). These include gain, frequency and phase delay.

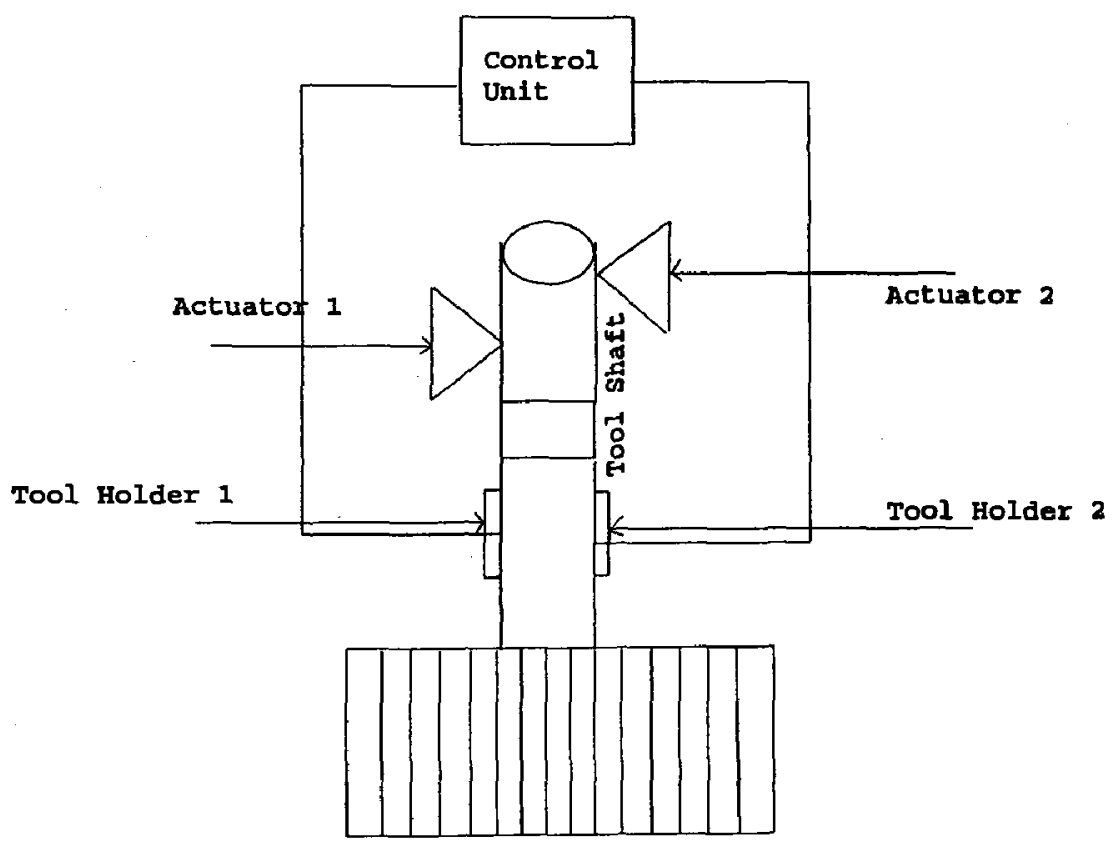

Figure 5.13: Simplified Milling Cutting Process with Two Actuators

\subsubsection{Off -Line Training}

Off-line training of the neural network was proposed using a training set containing 900 patterns. A computer program was written in $\mathrm{C}$ language to generate these patterns. The test set included 390 patterns not used during the training of the neural network. 
In order to avoid over training and have the best generalization capabilities the number of hidden nodes were chosen based on preference on test set. Figure 5.14 shows the result for various numbers of hidden nodes as obtained by using the test set. While the training error will decrease by increasing the number of hidden nodes the test set error will increase for hidden nodes more than 10. This indicates that for neural network with more than 10 hidden nodes, the NN start to over train and therefore loses some of the generalization capability.

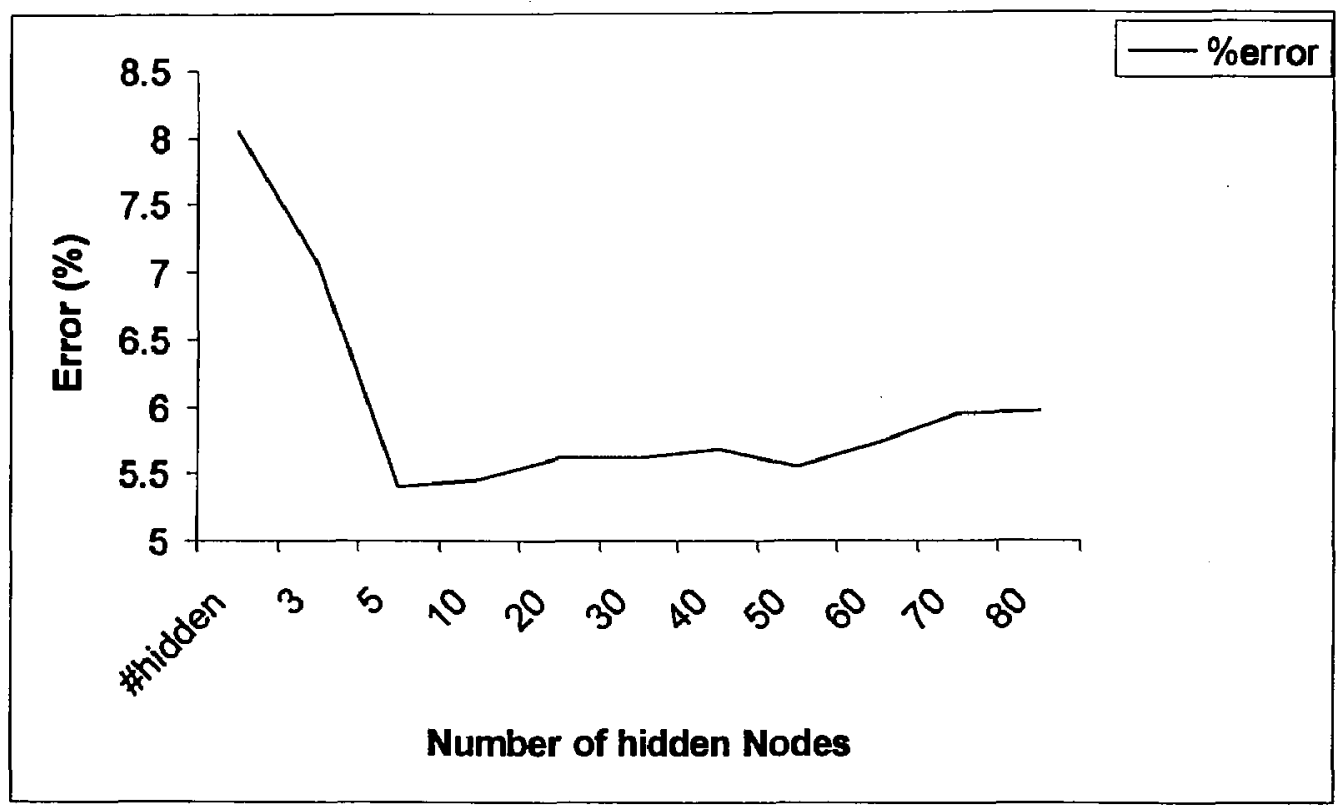

Figure 5.14: Neural Network with Varying Number of Hidden Nodes

\subsubsection{Simulation Results}

After achieving adequate results in off-line training the neural network model was used for simulation studies. Figure 5.15 shows the chatter in $x$ direction when a chip width of $b=3.63$ is used and the chatter suppression system is off. Figure 5.16 shows chatter although with a smaller amplitude in $y$ direction. The frequency response of the vibration in $\mathrm{x}$ axis is shown in Figure 5.17 and indicates a very sharp peak. 
The results when suppression system was activated are depicted in Figures 5.18 and 5.19. Figures 5.18 and 5.19 show the vibration in $x$ and $y$ direction when the chatter suppression system was activated. Although the vibration is not totally eliminated, the amplitude has been reduced. This can also be seen in Figure 5.20 which compared the frequency response before and after suppression system was activated.

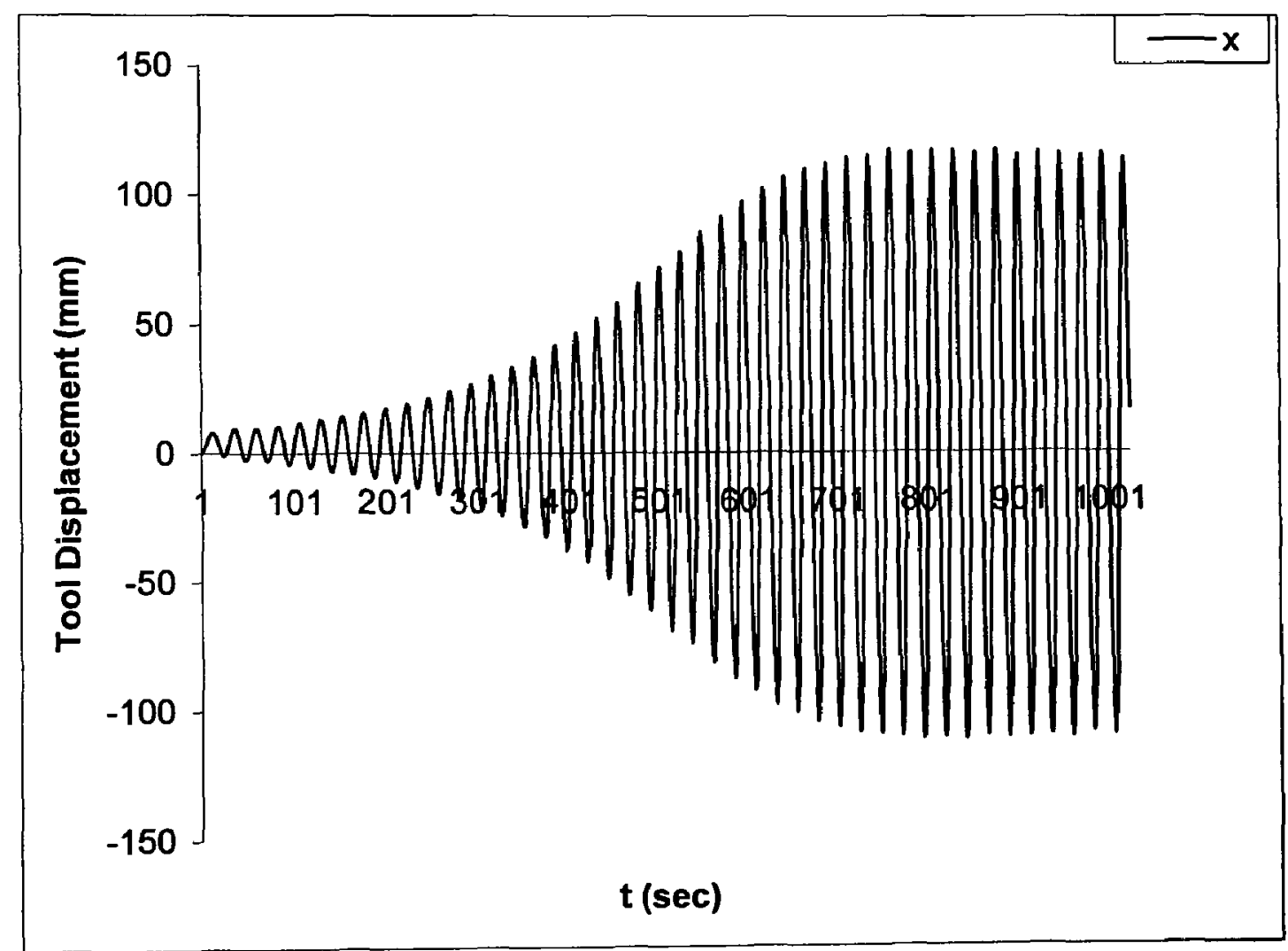

Figure 5.15: Tool chatter with active suppression system Off 


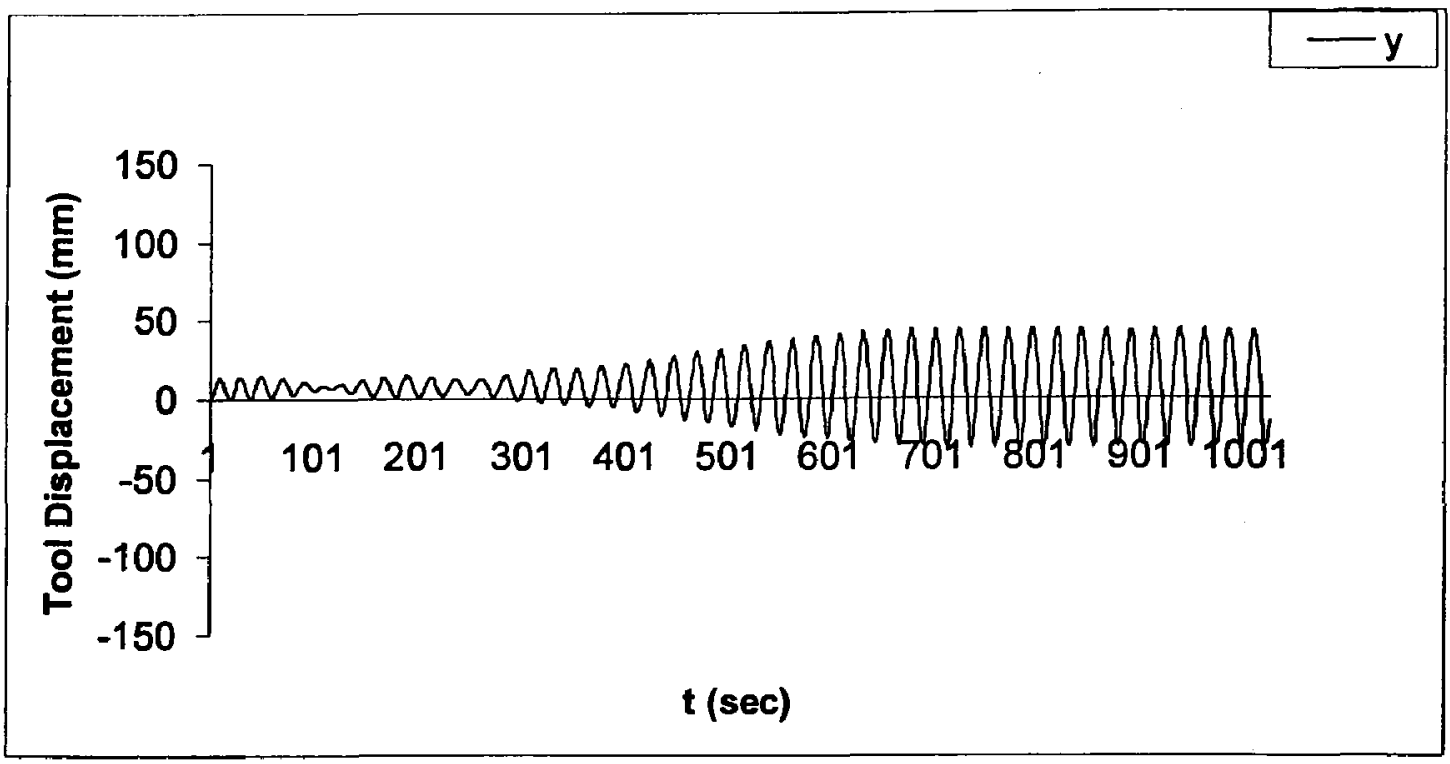

Figure 5.16: Tool chatter with active suppression system Off

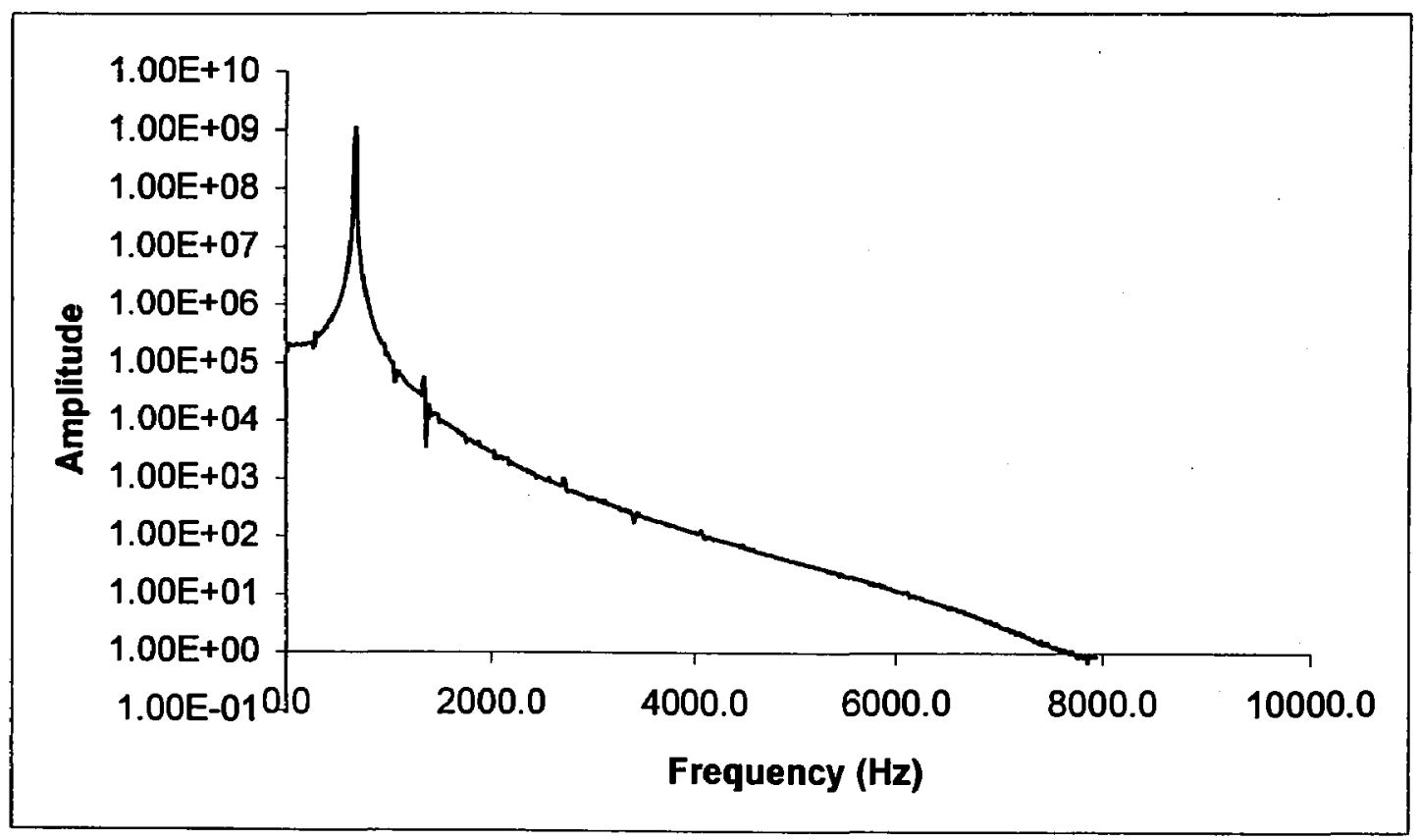

Figure 5.17: Frequency response of the tool with active suppression system off. 


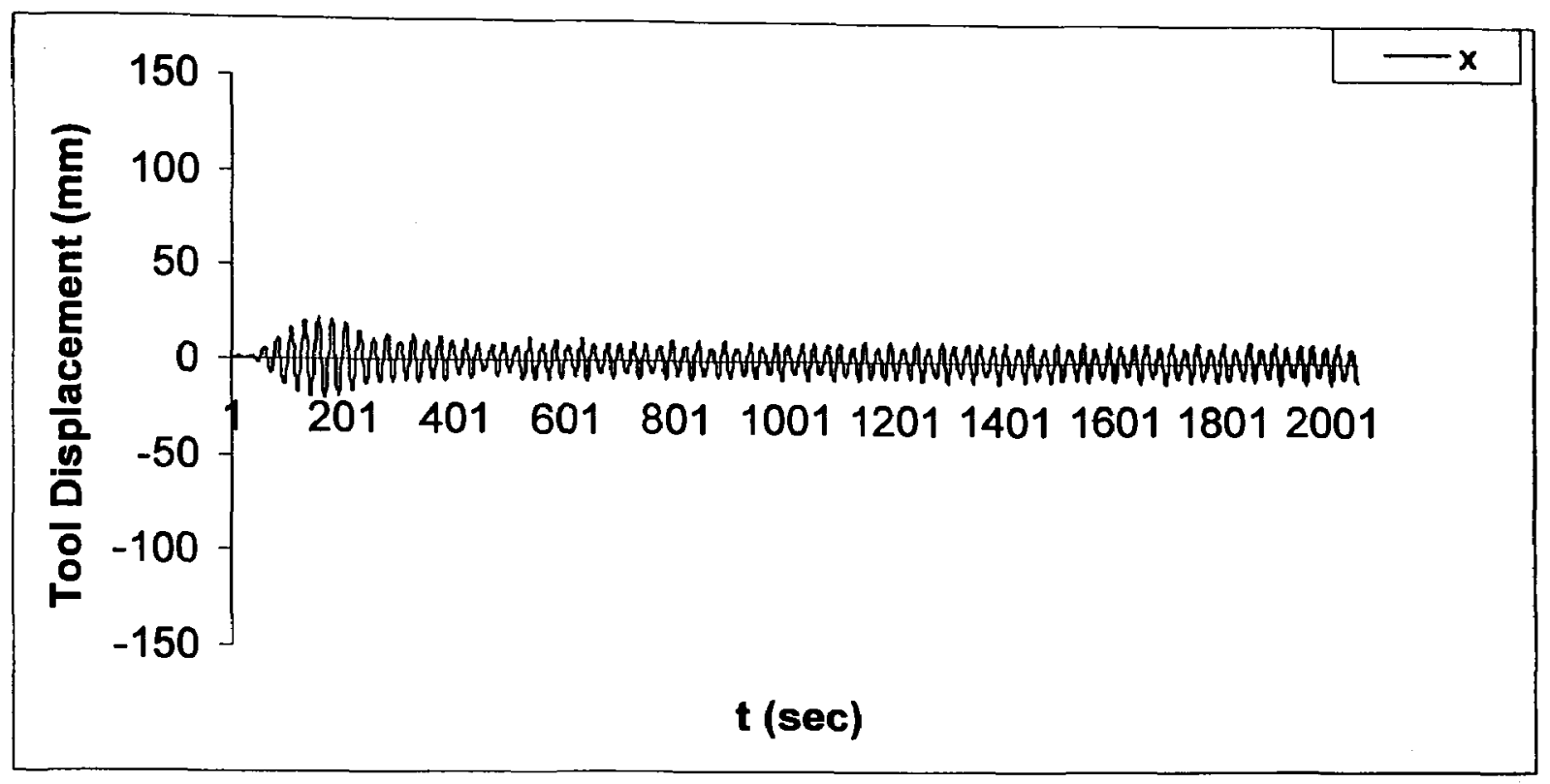

Figure 5.18: Tool vibration with active suppression system On

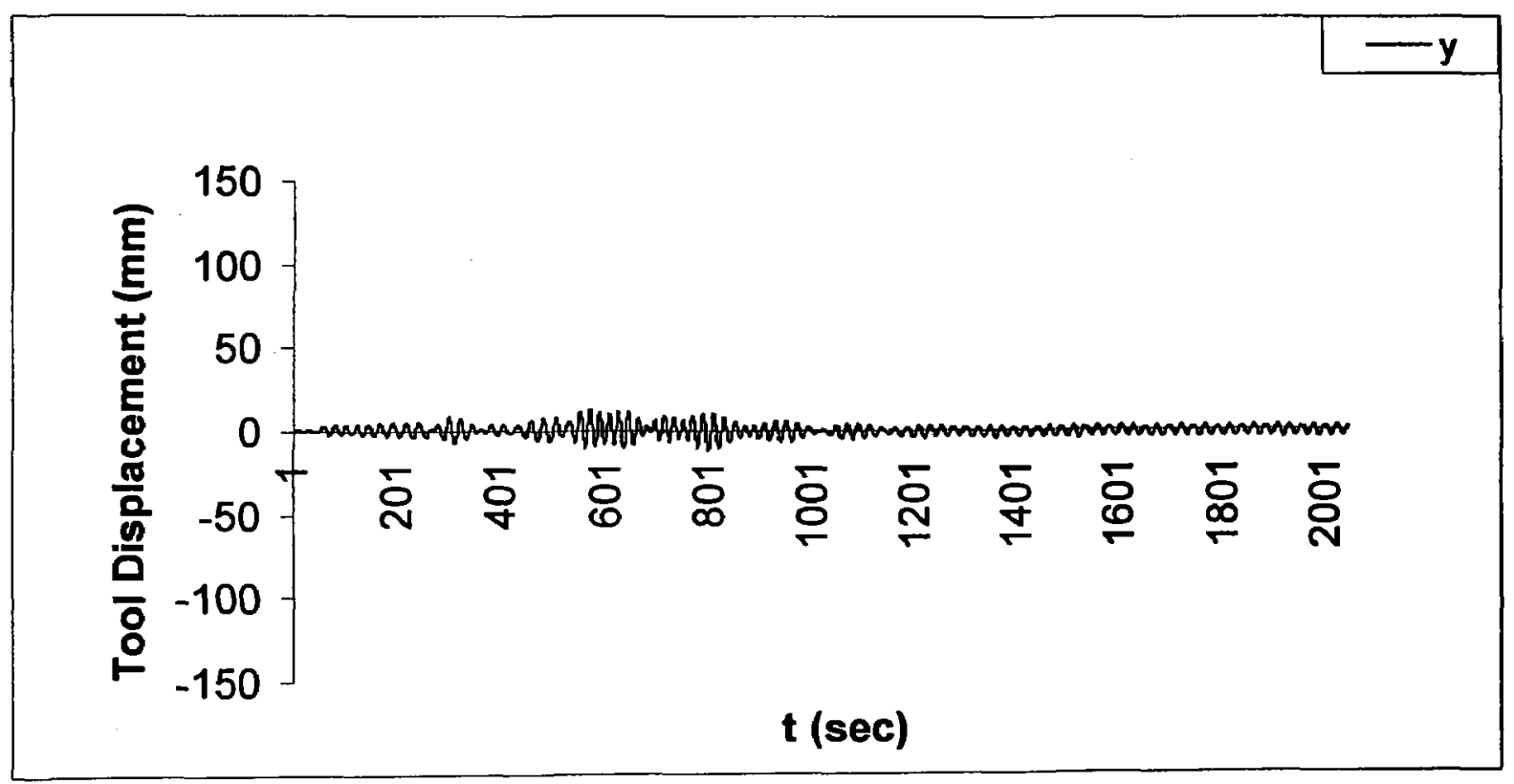

Figure 5.19: Tool vibration with active suppression system On 


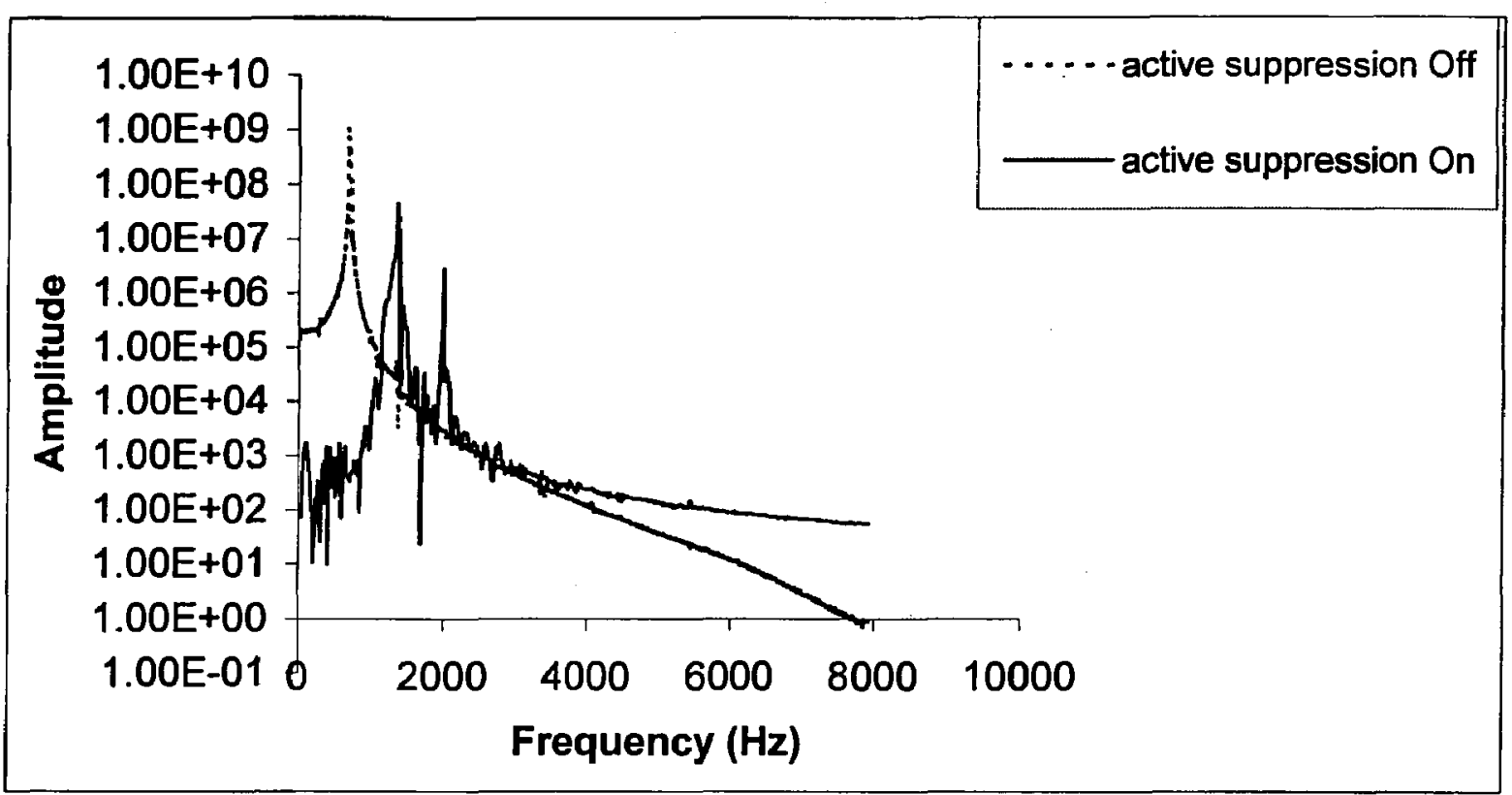

Figure 5.20: Comparison of frequency response of the tool with active suppression On and Off. 


\section{Chapter 6}

\section{Conclusions}

\subsection{Contributions}

A novel method for active method suppression of chatter in machining process has been developed. The proposed method can deal with non-linearities associated with the chatter process including time delays inherent in the processes. Using neural networks as a dynamic model, the chatter suppression system can adapt to changes in the cutting process, thus eliminating the need for repeated turning.

\subsection{Concluding Remarks}

The work described in this thesis was aimed at developing an on-line system for chatter suppressing in machining operations. Different methods of generating the training sets and different neural networks architecture were investigated. It was found that improvements in the error of the training and test sets could be achieved by randomizing the order in which data were presented to the system and by using fully normalized data. Normalizing the input data between the same upper and lower values as the output data ensures that all data is treated equally by the neural network.

It has been shown that through proper signal processing, judicious choice of architecture and application of appropriate training techniques, a neural network can be trained to correlate gradient calculation with respect to the parameters of the active vibration source with reasonable accuracy.

\subsection{Recommendations}

The work presented has shown that the chatter can be suppressed by using the proposed methodology. Although simulation results are encouraging, experimental verification is 
required. The challenges will arise from noise in the signals, unmodeled time delays and transverse chatter and interaction with radial chatter.

Future work should concentrate on improving the neural network model. The addition of an on-line network after the off-line trained neural network may be able to improve the quality of the model, and increase the effectiveness of the system. 


\section{REFERENCES}

[1] Industry Canada, Automotive and Transportation branch. Statistical review of the Canadian automotive industry: 1999 Edition. Ottawa, ON. 1999.

[2] Ismail, F and R. Ziaei. "Chatter Suppression in five -Axis Machining of Flexible Parts: International Journal of Machine tools \& Manufacture,2002, Vol.42, pp 115-122

[3] Rao, Balkrishna C and Yung C.Shin " A Comprehensive Dynamic cutting Force Model for Chatter Predicyion in Turning" International Journal of machine tools \& manufacture ,1999, Vol 39 ,pp. 1631- 1654

[4] Boothroyd , Geoffrey and Winston A Knight, 1989, Fundamentals of Machining Tools, $2^{\text {nd }}$ edition, Marcel Dekker, New York.

[5] Jiri (George) Tlusty, Manufacturing process and equipment, by prentice Hall, prentice Hall, Inc.NJ.2000, pp.559-574

[6] Chiriacescu, Sergiu T. "Stability in the dynamics of metal cutting", 1990,Elsevier Science Publication, Co.

[7] Lago, Thomas L., Sven Oisson, lars Hakansson and Ingavar Claesson " Performance of a chatter control system for turning and boring applications" $4^{\text {th }}$ GRACM congress on computational Mechanics,june 2002, pp.27-29

[8] Landers, Robert G and Galip Ulsoy. "Chatter Analysis of machining system with nonlinear force Processes" ASME International Mechanical Engineering Congress and Exposition Atlanta, Georgia, November 17-22, 1996, DSC Vol.58, pp.183-190

[9] Liao, Y.S and Y.C Young. "A new on-line spindle speed regulation strategy for chatter control" International Journal.Mach Tool Manufact, 1996, Vol. 36. No 5, pp. 651-660.

[10] Merrit, H.E, Journal of engineering for Industry, 1965, Vol 87, 447-454

[11] Kim, K.J and J.Y.Ha, "Suppression of machine tool chatter using a viscoelastic dynamic damper", Journal of Engineering for Industry, travsaction of ASME,February 1987, Vol.109,pp.58-64

[12] Xiao, M., Kanube, S., Soutome, T and Ka Sato, "Analysis of chatter suppression in vibration cutting" Internationl journal of machine tool \& manufacturing ,2002, Vol 42 pp.1677-1685 
[13] Tang, YS., Kao, J.Y and E.C .Lee "Chatter suppression in turning operations with a tuned vibration absorber", Journal of materials processing technology, 2000, Vol 105,pp $55-60$

[14] Slocum, Alexande H., Marsh, Eric $r$ and Douglas H. Smith. Massachusetts "A new damper design for the machine tool structure: the replicated internal viscous" Massachusetts Institute of Technology, Cambridge, MA, USA, July 1994, Vol 16, No 3

[15] Jang, J.L and Y.S. Tang "A study of the active vibration control of a cutting tool "Journal of materials processing technology, 1990, 95, pp 78-82

[16] Chiou, Chu-Hsiang., Hong, Min -Sung and Kornel F. Ehmann, "The feasibility of eigenstructure assignment for machining chatter control", International journal of machine tools \& manufacturer, 2003.Vol 3, pp. 1603-1620

[17] Mehrota, k., Mohan, C.K. and S.Ranka, "Elements of artificial neural networks" Cambridge, MA: MIT press, 1997

[18] Principe, Jose C., Euliano, Neil R and W.Curt Lafebvre, "Neural and Adaptive System" fundamentals through simulations, Publisher .John Wiley and S, Inc, 1999, pp. $100-132$

[19] Barron, AR. "Universal Approximation Bounds for Superpositions of a Sigmoidal Function.", IEEE Transaction on information theory.1993, 39, pp. 930-945

[20] T. Master, Practical Neural Recipes in C++. London,Academic press, 1993.

[21] D.Rumelhart and j. McClelland, "Parallel distributed processing" MIT press 1986, Vol 1, Cambridge MA.

[22] Rangwala, Sabbir S. and David a. Dornfeld. "Learning and optimization of machining operations using computing abilities of neural network", IEEE transaction on systems, man, and cybernetics, march/april 1989 vol 19, pp.2

[23] Press, W.H. Teulosky, Vetterling, S.A. and B.P. Flannery. "Numerical Recipes in CThe art of scientific computing. Melbourne: Cambridge University press, 1992.

[24] Akaike, H. " New look at the statistical model identification", IEEE Transaction on Automatic Control ,1974 Vol AC-19,n 6,pp. 716-723

[25] Hasting, Roy.K, Milling with NC, Deborn, Michigan, Society of manufacturing engineers, 1976, pp 63-65 


\section{Vita}

Name Israr Ahmed Siddiqui

Education Master of Applied Science 2004

Ryerson University, Toronto,ON. Canada

Major: Mechanical Engineering

Bachelor of engineering

1993

NED University of Engineering and Technology

Karachi,Pakistan

Major: Mechanical Engineering

Experience Aerospace Field Designer, 2001-2002

MTI - PolyFab, Mississauga, ONT

Project Engineer

1993-2001

Siemens Engineering Ltd, Karachi

Publications Conference paper 1: I.Siddiqui, A.Ghasempoor "Adaptive Feed forward chatter suppression in machining" 03AMTC-15, copyright@2003 SAE International

Conference paper2: "An Intelligent system for chatter suppression in machining"

Present in International conference -FAIM 2004 at Ryerson University on July $14^{\text {th }}, 2004$ 